\title{
On the maximum density of fixed strongly connected subtournaments
}

\author{
Leonardo N. Coregliano* \\ Institute of Mathematics and Statistics \\ Universidade de São Paulo \\ São Paulo, Brazil \\ lenacore@ime.usp.br
}

\author{
Roberto F. Parente ${ }^{\dagger}$ \\ Department of Computer Science \\ Universidade Federal da Bahia \\ Bahia, Brazil \\ roberto.parente@ufba.br
}

\author{
Cristiane M. Sato \\ Center of Mathematics, Computing and Cognition \\ Universidade Federal do ABC \\ São Paulo, Brazil \\ c.sato@ufabc.edu.br
}

Submitted: Oct 22, 2016; Accepted: Feb 19, 2019; Published: Mar 22, 2019

(C) The authors. Released under the CC BY-ND license (International 4.0).

\begin{abstract}
We study the density of fixed strongly connected subtournaments on 5 vertices in large tournaments. We determine the maximum density asymptotically for five tournaments as well as unique extremal sequences for each tournament. As a byproduct we also characterize tournaments that are recursive blow-ups of a 3-cycle as tournaments that avoid three specific tournaments of size 5 .
\end{abstract}

Mathematics Subject Classifications: 68R05, 05C20,05C35

\section{Introduction}

A locally transitive tournament is a tournament $T$ such that the outneighbourhood

$$
N^{+}(v)=\{w \in V(T): v w \in A(T)\}
$$

and the inneighbourhood $N^{-}(v)=\{w \in V(T): w v \in A(T)\}$ of every vertex $v \in V(T)$ are both transitive. Alternatively a locally transitive tournament is a tournament that

*Supported by Fundação de Amparo à Pesquisa do Estado de São Paulo (FAPESP) under grant no. $2013 / 23720-9$

${ }^{\dagger}$ Supported by CNPq (140987/2012-6) while a doctoral student at Universidade de São Paulo.

${ }^{\ddagger}$ Partially supported by FAPESP (Proc.2103/03447-6) 
has no occurrences of $W_{4}$ nor of $L_{4}$, where $W_{4}$ and $L_{4}$ are the tournaments of size 4 with outdegree sequences $(1,1,1,3)$ and $(0,2,2,2)$ respectively. On the other hand, a balanced tournament is a tournament with an odd number of vertices $2 n+1$ where each vertex has outdegree $n$. With these definitions, there is only one locally transitive balanced tournament $R_{2 n+1}$ of order $2 n+1$ up to isomorphism called carousel tournament (see Figure 1). This tournament is defined by $V\left(R_{2 n+1}\right)=\mathbb{Z}_{2 n+1}=\{0,1, \ldots, 2 n\}$ and

$$
A\left(R_{2 n+1}\right)=\left\{(v,(v+i) \bmod (2 n+1)): v \in V\left(R_{2 n+1}\right) \wedge i \in[n]\right\},
$$

where $[n]=\{1,2, \ldots, n\}$.

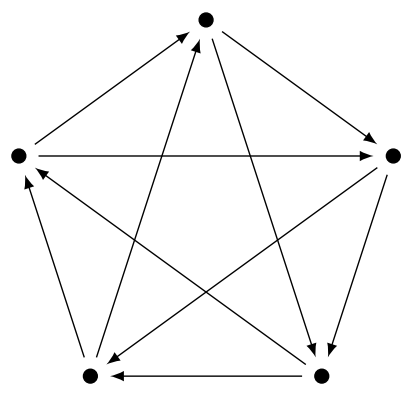

$R_{5}$

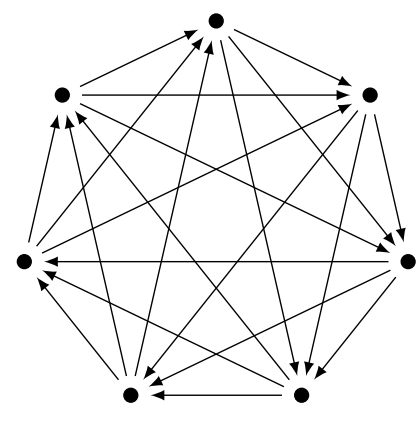

$R_{7}$

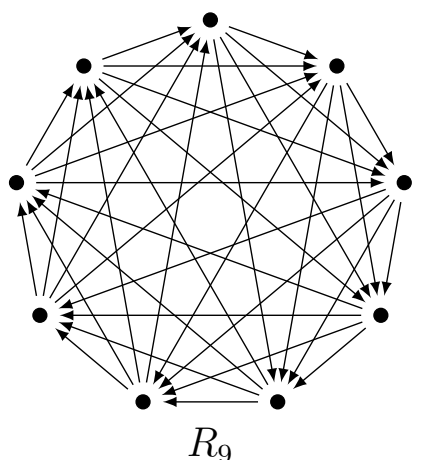

$R_{9}$

Figure 1: Carousel tournaments $R_{2 n+1}$ for $n=2,3,4$.

In 1964, Colombo [10] proved that the maximum number of oriented 4-cycles in a tournament of odd size is attained by the carousel tournament $R_{2 n+1}$. One year later, Beineke and Harary [3] extended this result by proving that the carousel tournament $R_{2 n+1}$ also maximizes the number of strongly connected subtournaments of a fixed size in a tournament of odd size ${ }^{1}$.

The problem of maximizing subtournaments in a tournament of fixed size is in general a hard problem and determining which tournaments are extremal is an even harder one. However, in many cases, the easier problem of maximizing the asymptotic density is completely solvable, that is, not only can we find the value of the maximum asymptotic density, but we can also characterize all extremal families of tournaments.

Such characterization of an asymptotically extremal family $\left(T_{n}\right)_{n \in \mathbb{N}}$ generally comes in the flavour of saying that $\left(T_{n}\right)_{n \in \mathbb{N}}$ converges to a certain "limit object", that is, for every fixed tournament $T$, we have

$$
\lim _{n \rightarrow \infty} p\left(T ; T_{n}\right)=\phi(T)
$$

where $p\left(T ; T_{n}\right)$ denotes the unlabelled density of $T$ in $T_{n}$ (which is a number in $\left.[0,1]\right)^{2}$.

\footnotetext{
${ }^{1}$ Both results also provided a maximizer for even size, but we refrain from defining it here.

${ }^{2}$ Of course that if $\left(T_{n}\right)_{n \in \mathbb{N}}$ maximizes the asymptotic density of $T$, then we will know the value of $\lim _{n \rightarrow \infty} p\left(T ; T_{n}\right)$, but the strength of this characterization is that extremality for $T$ forces the densities of other tournaments $T^{\prime} \neq T$ to converge to specific values.
} 
There are basically two approaches to defining what is a "limit object". The first approach is to define the limit object to be semantically close to the underlying object, which has been carried out successfully for several combinatorial objects such as graphs [25], uniform hypergraphs [17], digraphs [16, Section 9] $]^{3}$ and permutations [21]. The second approach is to define the limit object syntactically, that is, to study what sorts of properties must a sequence $(\phi(T))_{T}$ satisfy if it is obtained as $\forall T, \lim _{n \rightarrow \infty} p\left(T ; T_{n}\right)=\phi(T)$ for a sequence of objects $\left(T_{n}\right)_{n \in \mathbb{N}}$. This latter approach is precisely the thrust of the theory of flag algebras [28] and in what follows we will mostly use this language.

In this paper, we study the problem of maximizing the asymptotic density of a single fixed strongly connected tournament $T$ of size 5 , that is, we are interested in computing

$$
\lim _{n \rightarrow \infty} \max \left\{p\left(T ; T_{n}\right):\left|V\left(T_{n}\right)\right|=n\right\}
$$

for $T \in\left\{T_{5}^{7}, T_{5}^{8}, T_{5}^{9}, T_{5}^{10}, T_{5}^{11}, T_{5}^{12}\right\}$ (see Figure 2$)^{4}$ and characterizing sequences $\left(T_{n}\right)_{n \in \mathbb{N}}$ such that $\lim _{n \rightarrow \infty} p\left(T ; T_{n}\right)$ is equal to this value. Note that this differs from the result of Beineke and Harary in that they proved that for every $k \in \mathbb{N}$, we have

$$
\max \left\{\sum_{T \in \mathcal{S}_{k}} p\left(T ; T_{2 n+1}\right):\left|V\left(T_{2 n+1}\right)\right|=2 n+1\right\}=\sum_{T \in \mathcal{S}_{k}} p\left(T ; R_{2 n+1}\right),
$$

where $\mathcal{S}_{k}$ denotes the set of all strongly connected tournaments of size $k$.
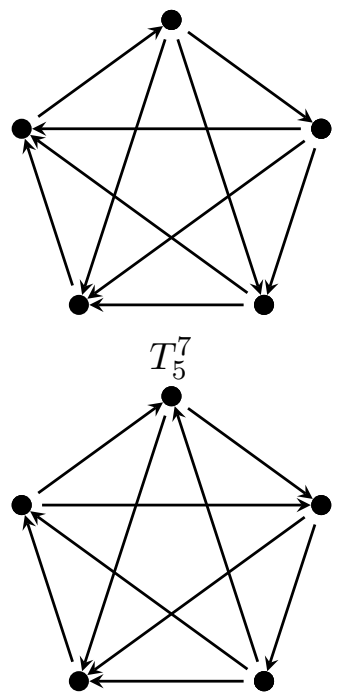

$T_{5}^{10}$
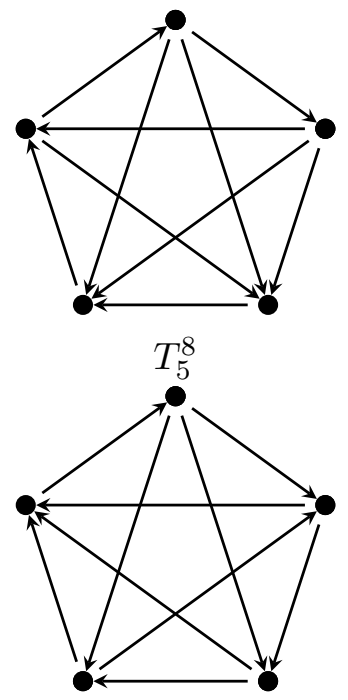

$T_{5}^{11}$
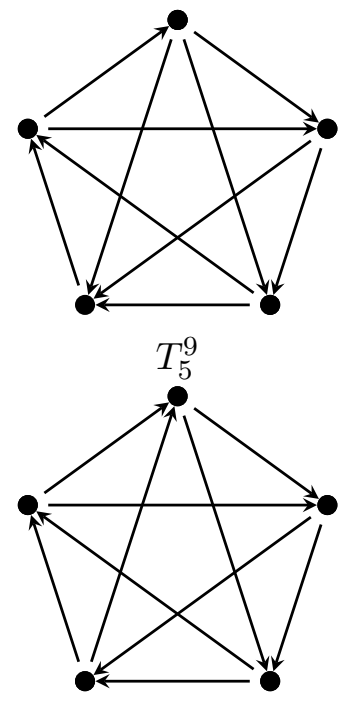

$T_{5}^{12}$

Figure 2: Strongly connected tournaments of size 5.

\footnotetext{
${ }^{3}$ As expected, the limit object for a tournament is just a special case of the limit object for a digraph.

${ }^{4}$ It is easy to see that the sequence of maximum values has decreasing tail, hence it is convergent.
} 
For the asymptotic study of tournaments, we have a weaker notion of balanced tournament. Namely a sequence of tournaments $\left(T_{n}\right)_{n \in \mathbb{N}}$ of increasing sizes is said to be asymptotically balanced if all except for $o\left(\left|T_{n}\right|\right)$ vertices of $T_{n}$ have outdegree $(1 / 2+o(1))\left|T_{n}\right|$.

In the theory of flag algebras, the notion of a limit object is captured by a positive homomorphism $\phi \in \operatorname{Hom}^{+}\left(\mathcal{A}^{0}, \mathbb{R}\right)$ (see Section 3.1) and we have the analogous notion of a balanced homomorphism, which a homomorphism that is the limit of an asymptotically balanced sequence of tournaments.

In this paper, we prove extremality theorems involving three important balanced homomorphisms.

The first homomorphism is the quasi-random homomorphism $\phi_{\mathrm{qr}}$, which is the main topic of study of the theory of tournament quasi-randomness.

For every $n \in \mathbb{N}$, let $\boldsymbol{R}_{\boldsymbol{n}, \mathbf{1 / 2}}$ be the random tournament of size $n$ where each arc orientation is present with probability $1 / 2$ independently of all other pairs of vertices. It is a straightforward exercise in binomial concentration to prove that with probability 1 we have

$$
\forall T \text { tournament, } \lim _{n \rightarrow \infty} p\left(T ; \boldsymbol{R}_{\boldsymbol{n}, \mathbf{1 / 2}}\right)=\mathbb{E}\left[p\left(T ; \boldsymbol{R}_{|\boldsymbol{T}| \mathbf{1} \mathbf{1} \mathbf{2}}\right)\right]=\frac{|T| !}{|\operatorname{Aut}(T)| 2^{\left(\begin{array}{c}
|T| \\
2
\end{array}\right)},}
$$

that is, the sequence $\left(\boldsymbol{R}_{\boldsymbol{n}, \mathbf{1 / 2}}\right)_{n \in \mathbb{N}}$ of random tournaments is convergent with probability 1 . The quasi-random homomorphism $\phi_{\mathrm{qr}}$ is then defined as the almost sure limit of this sequence.

The theory of quasi-randomness started with the study of quasi-random graphs in the seminal papers by Thomason [32] and Chung, Graham and Wilson [9] and now has branches in several other theories such as uniform hypergraphs $[7,6,4]$, graph orientations $[19]$, permutations $[11,23]$ and tournaments $[8,22,12]$. We do not attempt to provide a detailed review of the theory of quasi-randomness here (see [24] for a survey), but its main gist is that there are several a priori different properties of a homomorphism $\phi \in \operatorname{Hom}^{+}\left(\mathcal{A}^{0}, \mathbb{R}\right)$, called quasi-random properties, that force $\phi=\phi_{\mathrm{qr}}$.

For instance, an example of a quasi-random property was proven by the first author and Razborov [13]: for every $k \geqslant 4$, if $\phi$ minimizes the density of the transitive tournament of size 4 , then $\phi=\phi_{\mathrm{qr}}$.

Regarding $\phi_{\mathrm{qr}}$, we prove the following result.

Theorem 1. We have

$$
\lim _{n \rightarrow \infty} \max \left\{p\left(T_{5}^{8} ; T_{n}\right):\left|T_{n}\right|=n\right\}=\frac{15}{128} .
$$

Furthermore, if $\left(T_{n}\right)_{n \in \mathbb{N}}$ is a sequence of tournaments of increasing sizes, then

$$
\lim _{n \rightarrow \infty} p\left(T_{5}^{8}, T_{n}\right)=15 / 128
$$

if and only if $\left(T_{n}\right)_{n \in \mathbb{N}}$ is quasi-random, that is, if and only if $\left(T_{n}\right)_{n \in \mathbb{N}}$ converges to $\phi_{\mathrm{qr}}$. 
The second homomorphism studied is the carousel homomorphism $\phi_{\mathrm{R}}$, which is the limit of the sequence $\left(R_{2 n+1}\right)_{n \in \mathbb{N}}$ of carousel tournaments. Analogously to quasi-random properties, the quasi-carousel properties [12] are properties of a homomorphism $\phi \in$ $\operatorname{Hom}^{+}\left(\mathcal{A}^{0}, \mathbb{R}\right)$ that force $\phi=\phi_{\mathrm{R}}$.

In analogy with a locally transitive tournament, a homomorphism $\phi \in \operatorname{Hom}^{+}\left(\mathcal{A}^{0}, \mathbb{R}\right)$ satisfying $\phi\left(W_{4}+L_{4}\right)=0$ is called locally transitive. Perhaps the most important quasicarousel property says that $\phi_{\mathrm{R}}$ is the only homomorphism that is both balanced and locally transitive.

In this paper, we prove the following result involving $\phi_{\mathrm{R}}$.

Theorem 2. We have

$$
\begin{aligned}
& \lim _{n \rightarrow \infty} \max \left\{p\left(T_{5}^{7} ; T_{n}\right):\left|T_{n}\right|=n\right\}=\frac{5}{16} \\
& \lim _{n \rightarrow \infty} \max \left\{p\left(T_{5}^{12} ; T_{n}\right):\left|T_{n}\right|=n\right\}=\frac{1}{16} .
\end{aligned}
$$

Furthermore, a sequence of tournaments $\left(T_{n}\right)_{n \in \mathbb{N}}$ of increasing sizes is extremal for any of $T_{5}^{7}$ or $T_{5}^{12}$ if and only if it is quasi-carousel, that is, if and only if $\left(T_{n}\right)_{n \in \mathbb{N}}$ converges to $\phi_{\mathrm{R}}$.

We remark that (1) confirms the following conjecture proposed by the first author in [12] for the case $n=2$.

Conjecture 3. For every $n \geqslant 2$, a homomorphism $\phi \in \operatorname{Hom}^{+}\left(\mathcal{A}^{0}, \mathbb{R}\right)$ maximizes $\phi\left(R_{2 n+1}\right)$ if and only if $\phi=\phi_{\mathrm{R}}$.

Finally, the last homomorphism studied is what we call here triangular homomorphism $\phi_{\vec{C}_{3}}$.

To define $\phi_{\vec{C}_{3}}$, we must first define recursive blow-ups of the 3-cycle $\vec{C}_{3}$. For every $n \geqslant$ 3 , let $n_{0} \geqslant n_{1} \geqslant n_{2}$ be such that $n_{0}+n_{1}+n_{2}=n$ and $n_{i} \in\{\lfloor n / 3\rfloor,\lceil n / 3\rceil\}$ for all $i \in$ $\{0,1,2\}$. Define $A_{0}=\left\{1, \ldots, n_{0}\right\}, A_{1}=\left\{n_{0}+1, \ldots, n_{1}\right\}$ and $A_{2}=\left\{n_{1}+1, \ldots, n_{2}\right\}$. Let $\vec{C}_{n}^{3}$ be the tournament on $[n]$ such that $v w \in A\left(\vec{C}_{n}^{3}\right)$ for every $v \in A_{i}$ and $w \in A_{(i+1) \bmod 3}$, and $\left.\vec{C}_{n}^{3}\right|_{A_{i}}$ is isomorphic to $\vec{C}_{n_{i}}^{3}$ for every $i=0,1,2$ (see Figure 3 ).

We define then $\phi_{\vec{C}_{3}}$ as the limit of the sequence $\left(\vec{C}_{n}^{3}\right)_{n \in \mathbb{N}}$ (see Proposition 45 for the convergence of this sequence).

Our final extremality result concerns $\phi_{\vec{C}_{3}}$.

Theorem 4. We have

$$
\begin{aligned}
& \lim _{n \rightarrow \infty} \max \left\{p\left(T_{5}^{9} ; T_{n}\right):\left|T_{n}\right|=n\right\}=\frac{3}{8} \\
& \lim _{n \rightarrow \infty} \max \left\{p\left(T_{5}^{11} ; T_{n}\right):\left|T_{n}\right|=n\right\}=\frac{1}{16} .
\end{aligned}
$$

Furthermore, a sequence of tournaments $\left(T_{n}\right)_{n \in \mathbb{N}}$ of increasing sizes is extremal for any of $T_{5}^{9}$ or $T_{5}^{11}$ if and only if it is quasi-triangular, that is, if and only if $\left(T_{n}\right)_{n \in \mathbb{N}}$ converges to $\phi_{\vec{C}_{3}}$. 


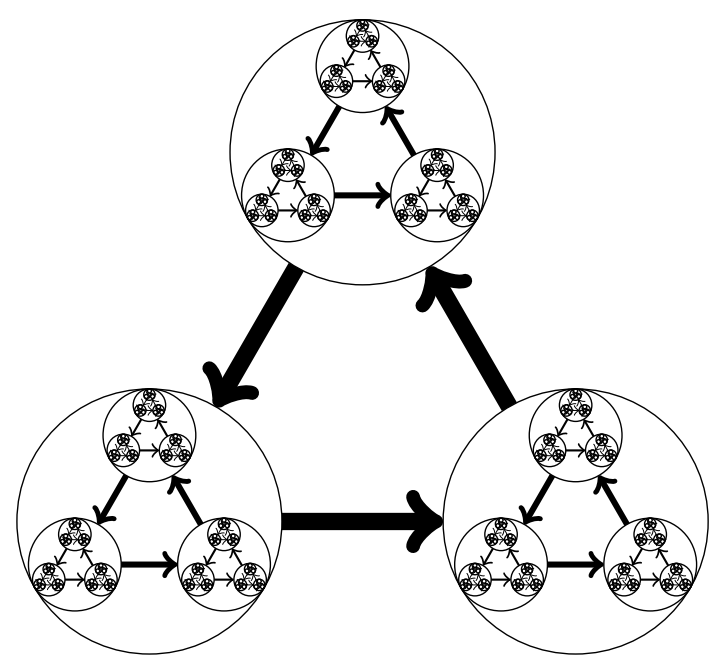

Figure 3: Typical structure of $\vec{C}_{n}^{3}$.

Motivated by the definition of $\vec{C}_{n}^{3}$, we say that a tournament is $\vec{C}_{3}$-decomposable (see Definition 31) if it has the same structure of $\vec{C}_{n}^{3}$ but without requiring the parts to be as equal as possible. And, as an auxiliary result, we prove (Theorem 33) that a tournament is $\vec{C}_{3}$-decomposable if and only if it has no copies of $T_{5}^{8}, T_{5}^{10}$ nor of $T_{5}^{12}$. We then extend this notion to homomorphisms by saying that a homomorphism $\phi \in \operatorname{Hom}^{+}\left(\mathcal{A}^{0}, \mathbb{R}\right)$ is $\vec{C}_{3^{-}}$ decomposable if $\phi\left(T_{5}^{8}+T_{5}^{10}+T_{5}^{12}\right)=0$.

As a byproduct of Theorem 4, we prove several quasi-triangular properties (i.e., properties of a homomorphism $\phi \in \operatorname{Hom}^{+}\left(\mathcal{A}^{0}, \mathbb{R}\right)$ that force $\left.\phi=\phi_{\vec{C}_{3}}\right)$. One of them, namely $L_{4}$, says that $\phi_{\vec{C}_{3}}$ is the only homomorphism that is both balanced and $\vec{C}_{3}$-decomposable.

Let us finally remark that our results fail to cover only one strongly connected tournament of size 5 , which is $T_{5}^{10}$.

The paper is organized as follows. We prove the lower bounds of Theorems 1, 2 and 4 in Section 2. The proof of the upper bounds are given in Section 4 and are an application of Razborov's semidefinite method for flag algebras [29] (see also [2, 14, 15, 18, 27] for some examples). In Section 3, we present a brief overview of flag algebras and this method. The uniqueness proofs are presented in Section 6. In Section 5, we show how to extract informations about extremal sequences from the semidefinite method. We postpone the proof of the characterization of $\vec{C}_{3}$-decomposable tournaments to Section 7 and postpone the proof of a technical lemma on quasi-triangular properties to Section 8 .

\section{Lower bounds}

In this section, we prove the lower bounds in Theorems 2,1 and 4 .

We start by recalling the definition of labelled density in tournaments.

Definition 5. If $T_{1}$ and $T_{2}$ are tournaments with $\left|T_{1}\right| \leqslant\left|T_{2}\right|$, then the labelled density 
of $T_{1}$ in $T_{2}$ (denoted $t_{\text {ind }}\left(T_{1} ; T_{2}\right)$ ) is the probability that an injective mapping from $V\left(T_{1}\right)$ to $V\left(T_{2}\right)$ picked uniformly at random is an embedding of $T_{1}$ in $T_{2}$.

It is easy to see that

$$
t_{\text {ind }}\left(T_{1} ; T_{2}\right)=\frac{\left|\operatorname{Aut}\left(T_{1}\right)\right|}{\left|T_{1}\right| !} p\left(T_{1} ; T_{2}\right)
$$

where $\operatorname{Aut}\left(T_{1}\right)$ is the group of automorphisms of $T_{1}$.

Lemma 6. We have

$$
\lim _{n \rightarrow \infty} p\left(T_{5}^{7} ; R_{2 n+1}\right)=\frac{5}{16} ; \quad \quad \lim _{n \rightarrow \infty} p\left(T_{5}^{12} ; R_{2 n+1}\right)=\frac{1}{16} .
$$

Proof. We will prove only the assertion for $T_{5}^{12}$, since the proof for $T_{5}^{7}$ is very similar.

Fix $n \geqslant 2$ and let $f: V\left(R_{5}\right) \rightarrow V\left(R_{2 n+1}\right)$ be an embedding of $R_{5}$ in $R_{2 n+1}$.

Suppose that the vertex 0 from $R_{5}$ is mapped to the vertex 0 of $R_{n}$. If vertex 1 is mapped to a vertex $i$, then $1 \leqslant i \leqslant n$ and vertex 2 has to be mapped to a vertex $j$ such that $i+1 \leqslant j \leqslant n$. Vertex 3 has to be mapped to a vertex $k$ such that $n+1 \leqslant k \leqslant i+n$ (since $(3,0)$ and $(1,3)$ are arcs of $\left.R_{5}\right)$. Finally, vertex 4 has to be mapped to a vertex $\ell$ such that $i+n \leqslant \ell \leqslant j+n$ (since $(4,1)$ and $(2,4)$ are arcs of $\left.R_{5}\right)$. See Figure 4.

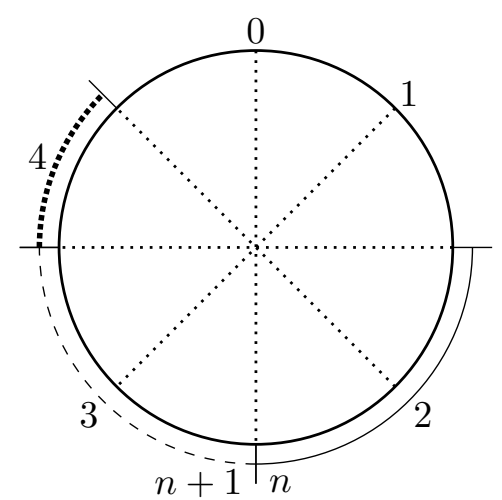

Figure 4: Possibilities of embedding $T_{5}^{12}=R_{5}$ in $R_{2 n+1}$.

Note that, after we fix the images of the vertices 0,1 and 2, the number of choices for the vertex 3 becomes $i$ and for the vertex 4 becomes $j-i+1$.

From the symmetry of $R_{2 n+1}$, we know that this is also the case for every other choice of the image of the vertex 0 of $R_{5}$. Thus, we have

$$
\begin{aligned}
t_{\text {ind }}\left(R_{5} ; R_{2 n+1}\right) & =\frac{1}{(2 n+1)_{5}} \cdot(2 n+1) \sum_{i=1}^{n} \sum_{j=i+1}^{n} i(j-i+1) \\
& =\frac{1}{(2 n)_{4}} \sum_{i=1}^{n} \frac{i^{3}-(2 n-2) i^{2}+n(n-2) i}{2}
\end{aligned}
$$




$$
\begin{aligned}
& =\frac{1}{(2 n)_{4}}\left(\frac{n^{4}}{24}+O\left(n^{3}\right)\right) \\
& =\frac{1}{3 \cdot 2^{7}}+O\left(\frac{1}{n}\right)
\end{aligned}
$$

where $(\ell)_{k}=\ell(\ell-1) \cdots(\ell-k+1)$ denotes the falling factorial.

Therefore

$$
\lim _{n \rightarrow \infty} p\left(T_{5}^{12} ; R_{2 n+1}\right)=\lim _{n \rightarrow \infty} \frac{5 !}{5} t_{\text {ind }}\left(R_{5} ; R_{n}\right)=\frac{1}{16} .
$$

We now prove the lower bounds in Theorem 4 .

Lemma 7. We have

$$
\lim _{n \rightarrow \infty} p\left(T_{5}^{9} ; \vec{C}_{3^{n}}^{3}\right)=\frac{3}{8} ; \quad \quad \lim _{n \rightarrow \infty} p\left(T_{5}^{11} ; \vec{C}_{3^{n}}^{3}\right)=\frac{1}{16} .
$$

Proof. Again, we will prove only the assertion for $T_{5}^{9}$, since the proof for $T_{5}^{11}$ is very similar.

Let $T$ denote the tournament in Figure 5, which is isomorphic to $T_{5}^{9}$.

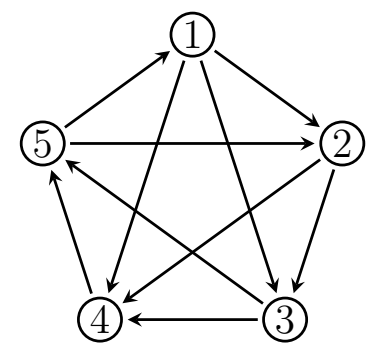

Figure 5: Tournament isomorphic to $T_{5}^{9}$.

Recall the definition of $\vec{C}_{3^{n}}^{3}$ and let $A_{0}=\left[3^{n-1}\right], A_{1}=\left\{3^{n-1}+1,3^{n-1}+2, \ldots, 2 \cdot 3^{n-1}\right\}$ and $A_{2}=\left\{2 \cdot 3^{n-1}+1,2 \cdot 3^{n-1}+2, \ldots, 3^{n}\right\}$.

Let $F(n)$ be the number of embeddings of $T$ in $\vec{C}_{3^{n}}^{3}$. Every such embedding either maps all vertices of $T$ to a single $A_{i}$ or it maps 1 and 5 to some part $A_{i}, 3$ and 4 to $A_{(i+1) \bmod 3}$

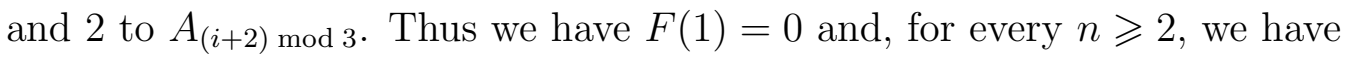

$$
F(n)=3\left(\begin{array}{c}
3^{n-1} \\
2
\end{array}\right)^{2} 3^{n-1}+3 F(n-1) \leqslant \frac{3^{5 n-4}}{4}+3 F(n-1) .
$$

Therefore, it follows that

$$
F(n) \leqslant \sum_{i=0}^{n-1} 3^{i} \frac{3^{5(n-i)-4}}{4}=\frac{3^{5 n-4}}{4} \cdot \frac{1-3^{-4 n}}{1-3^{-4}}=\frac{3^{5 n}}{320}+O\left(3^{4 n}\right) .
$$


On the other hand, we have

$$
F(n) \geqslant \frac{3^{5 n-4}-3^{4 n-2}}{4}+3 F(n-1),
$$

hence

$$
F(n) \geqslant \frac{3^{5 n}}{320}-\sum_{i=0}^{n-1} 3^{i} \frac{3^{2(n-i)-1}}{4}-O\left(3^{4 n}\right)=\frac{3^{5 n}}{320}-O\left(3^{4 n}\right)
$$

Therefore

$$
\lim _{n \rightarrow \infty} p\left(T_{5}^{9} ; \vec{C}_{3^{n}}^{3}\right)=\lim _{n \rightarrow \infty} \frac{F(n)}{\left(3^{n}\right)_{5}} \cdot 5 !=\frac{3}{8} .
$$

Finally, we prove the lower bound for $T_{5}^{8}$ in Theorem 1.

Lemma 8. We have

$$
\lim _{n \rightarrow \infty} \mathbb{E}\left[p\left(T_{5}^{8} ; \boldsymbol{R}_{\boldsymbol{n}, \mathbf{1 / 2}}\right)\right]=\frac{15}{128} .
$$

Proof. From the definition of $\boldsymbol{R}_{\boldsymbol{n}, \mathbf{1} / \mathbf{2}}$, it follows that

$$
\mathbb{E}\left[t_{\text {ind }}\left(T_{5}^{8} ; \boldsymbol{R}_{\boldsymbol{n}, \mathbf{1} / \mathbf{2}}\right)\right]=\frac{1}{2^{10}},
$$

for every $n \geqslant 5$, hence

$$
\lim _{n \rightarrow \infty} \mathbb{E}\left[p\left(T_{5}^{8} ; \boldsymbol{R}_{\boldsymbol{n}, \mathbf{1 / 2}}\right)\right]=\frac{1}{2^{10}} \cdot 5 !=\frac{15}{128} .
$$

\section{Razborov's semidefinite method for flag algebras}

In this section, we briefly review the basics of the flag algebra theory and its semidefinite method. Although we work here only with the theory of tournaments, we remark that flag algebras can be defined in the general setting of any universal theory of first-order (see [28] and [29], see also the surveys [30] and [31]).

\subsection{Basic definitions and properties}

First recall the definition of $\mathcal{T}_{n}$ as the set of all tournaments of on $n$ vertices up to isomorphism and define $\mathcal{T}=\bigcup_{n \in \mathbb{N}} \mathcal{T}_{n}$ as the set of all tournaments up to isomorphism on a finite number of vertices. For every tournament $T$, we will denote its size by $|T|=|V(T)|$.

A type is a tournament with vertex set $[k]=\{1,2, \ldots, k\}$ for some $k \in \mathbb{N}$ and, given a type $\sigma$ of size $k=|\sigma|$, a $\sigma$-flag is a partially labelled tournament such that the labelled part is a copy of $\sigma$. Formally, a $\sigma$-flag is a pair $(T, \theta)$, where $T$ is a tournament and $\theta:[k] \rightarrow V(T)$ is an embedding of $\sigma$ into $T$, that is, the function $\theta$ is an isomorphism 
between $\sigma$ and the tournament induced by $\operatorname{im}(\theta)$ on $T$. We define the size of the $\sigma$ flag $F=(T, \theta)$ as $|F|=|T|$.

We extend the notion of isomorphism to $\sigma$-flags declaring that a function $f: V\left(T_{1}\right) \rightarrow$ $V\left(T_{2}\right)$ is an isomorphism between the flags $F_{1}=\left(T_{1}, \theta_{1}\right)$ and $F_{2}=\left(T_{2}, \theta_{2}\right)$ if it is an isomorphism between $T_{1}$ and $T_{2}$ and $f \circ \theta_{1}=\theta_{2}$ (i.e., the function $f$ preserves labels). Naturally, we say that two flags $F_{1}$ and $F_{2}$ are isomorphic (denoted $F_{1} \cong F_{2}$ ) if there exists an isomorphism between them.

This allows us to define $\mathcal{F}_{n}^{\sigma}$ as the set of all $\sigma$-flags of size $n$ up to isomorphism and $\mathcal{F}^{\sigma}=\bigcup_{n \in \mathbb{N}} \mathcal{F}_{n}^{\sigma}$ as the set of all finite $\sigma$-flags up to isomorphism.

Let us denote the unique type of size 0 by 0 and note that a 0 -flag can be identified with a tournament. Let us also note that for every type $\sigma$, the set $\mathcal{F}_{|\sigma|}^{\sigma}$ has only one element $\left(\sigma\right.$, id), which we will denote by $1_{\sigma}$.

If $F=(T, \theta)$ is a $\sigma$-flag and $W \subset V(T)$ is such that $\operatorname{im}(\theta) \subset W$ (i.e., the set $W$ contains all labelled vertices), then we define the subflag induced by $W$ on $F$ as the flag $\left.F\right|_{W}=\left(\left.T\right|_{W}, \theta\right)$, where $\left.T\right|_{W}$ is the subtournament induced by $W$ on $T$.

We now extend the notion of density to flags as well and also to a more general setting of density of several flags.

Definition 9. Let $\sigma$ be a type of size $k$ and $\ell, \ell_{1}, \ell_{2}, \ldots, \ell_{t} \geqslant k$ be integers such that

$$
\left(\sum_{i=1}^{t} \ell_{i}\right)-(t-1) k \leqslant \ell .
$$

Let also $F=(M, \theta), F_{1}, F_{2}, \ldots, F_{t} \in \mathcal{F}^{\sigma}$ be $\sigma$-flags of sizes $\ell, \ell_{1}, \ell_{2}, \ldots, \ell_{t}$ respectively.

The joint density of $F_{1}, F_{2}, \ldots, F_{t}$ in $F$, denoted by $p\left(F_{1}, F_{2}, \ldots, F_{t} ; F\right)$, is defined through the following random experiment.

Pick uniformly at random pairwise disjoint subsets $\boldsymbol{W}_{\mathbf{1}}, \boldsymbol{W}_{\mathbf{2}}, \ldots, \boldsymbol{W}_{\boldsymbol{t}}$ of $V(F) \backslash \operatorname{im}(\theta)$ subject to $\left|\boldsymbol{W}_{\boldsymbol{i}}\right|=\ell_{i}-k$ for every $i \in[t]$ and define

$$
p\left(F_{1}, F_{2}, \ldots, F_{t} ; F\right)=\mathbb{P}\left[\forall i \in[t],\left.F\right|_{\operatorname{im}(\theta) \cup \boldsymbol{W}_{i}} \cong F_{i}\right] .
$$

We also extend $p$ linearly in each of its coordinates.

We can (finally) present the flag algebra of a type $\sigma$.

Proposition 10 (Razborov [28, Lemma 2.4]). Let $\sigma$ be a type of size $k$ and $\mathcal{A}^{\sigma}=\mathbb{R} \mathcal{F}^{\sigma} / \mathcal{K}^{\sigma}$ denote the quotient of the set $\mathbb{R} \mathcal{F}^{\sigma}$ of all formal linear combinations of elements of $\mathcal{F}^{\sigma}$ by the linear subspace $\mathcal{K}^{\sigma}$ generated by elements of the form

$$
\widetilde{F}-\sum_{F \in \mathcal{F}_{\ell}^{\sigma}} p(\widetilde{F} ; F) F
$$

where $\ell \geqslant|\widetilde{F}|$.

Define also the linear product $\cdot: \mathcal{A}^{\sigma} \times \mathcal{A}^{\sigma} \rightarrow \mathcal{A}^{\sigma}$ through

$$
F_{1} \cdot F_{2}=\sum_{F \in \mathcal{F}_{\ell}^{\sigma}} p\left(F_{1}, F_{2} ; F\right) F
$$


where $F_{1}, F_{2} \in \mathcal{F}^{\sigma}$ and $\ell \geqslant\left|F_{1}\right|+\left|F_{2}\right|-k$.

Under these conditions, this product is well-defined and the set $\mathcal{A}^{\sigma}$ equipped with this product (and the usual addition) is a commutative associative algebra over $\mathbb{R}$ with unity $1_{\sigma}$.

Let us denote by $\operatorname{Hom}\left(\mathcal{A}^{\sigma}, \mathbb{R}\right)$ the set of all $\mathbb{R}$-algebra homomorphisms from $\mathcal{A}^{\sigma}$ to $\mathbb{R}$ and define the set of positive homomorphisms as

$$
\operatorname{Hom}^{+}\left(\mathcal{A}^{\sigma}, \mathbb{R}\right)=\left\{\phi \in \operatorname{Hom}\left(\mathcal{A}^{\sigma}, \mathbb{R}\right): \forall F \in \mathcal{F}^{\sigma}, \phi(F) \in[0,1]\right\} .
$$

We will now define the notion of a convergent sequence of flags.

Definition 11. Let $\left(F_{n}\right)_{n \in \mathbb{N}}$ be a sequence of $\sigma$-flags.

The sequence $\left(F_{n}\right)_{n \in \mathbb{N}}$ is called increasing if $\left|F_{n}\right|<\left|F_{n+1}\right|$ for every $n \in \mathbb{N}$.

The sequence $\left(F_{n}\right)_{n \in \mathbb{N}}$ is called convergent if it is increasing and for every fixed $\sigma$ flag $F \in \mathcal{F}^{\sigma}$, the sequence $\left(p\left(F ; F_{n}\right)\right)_{n \in \mathbb{N}}$ is convergent.

If $\phi \in \operatorname{Hom}^{+}\left(\mathcal{A}^{\sigma}, \mathbb{R}\right)$ is a homomorphism, we say that the sequence $\left(F_{n}\right)_{n \in \mathbb{N}}$ converges to $\phi$ if it is convergent and

$$
\lim _{n \rightarrow \infty} p\left(F ; F_{n}\right)=\phi(F)
$$

for every $\sigma$-flag $F \in \mathcal{F}^{\sigma}$.

It is easy to see (e.g., by a diagonalization argument) that every increasing sequence of flags has a convergent subsequence. The next theorem says that the set of positive homomorphisms $\mathrm{Hom}^{+}\left(\mathcal{A}^{\sigma}, \mathbb{R}\right)$ captures precisely the limits of convergent sequences of $\sigma$ flags.

Theorem 12 (Lovász-Szegedy [25], Razborov [28, Theorem 3.3]). Every convergent sequence of $\sigma$-flags converges to a positive homomorphism in $\operatorname{Hom}^{+}\left(\mathcal{A}^{\sigma}, \mathbb{R}\right)$ and for every positive homomorphism $\phi \in \operatorname{Hom}^{+}\left(\mathcal{A}^{\sigma}, \mathbb{R}\right)$ there exists a sequence of $\sigma$-flags converging to $\phi$.

Recall that we are interested in maximizing the density of a fixed tournament $T$ asymptotically. This means that, in the language of flag algebras, we are interested in the following problem.

Problem 13. Given a fixed tournament $T \in \mathcal{T}$, compute

$$
\max \left\{\phi(T): \phi \in \operatorname{Hom}^{+}\left(\mathcal{A}^{\sigma}, \mathbb{R}\right)\right\} .
$$

Remark 14 . Here, we used max instead of sup because $\operatorname{Hom}^{+}\left(\mathcal{A}^{\sigma}, \mathbb{R}\right)$ is compact. 


\subsection{Semidefinite method}

Providing lower bounds to Problem 13 is easy. Indeed, every increasing sequence of tournaments $\left(T_{n}\right)_{n \in \mathbb{N}}$ provides the lower bound

$$
\limsup _{n \rightarrow \infty} p\left(T ; T_{n}\right)
$$

The hard part of this problem is to compute upper bounds. A first and naïve way of doing so is the following. If $\phi \in \operatorname{Hom}^{+}\left(\mathcal{A}^{\sigma}, \mathbb{R}\right)$ is a homomorphism, then Proposition 10 gives us

$$
\begin{aligned}
\phi(T) & =\sum_{T^{\prime} \in \mathcal{T}_{\ell}} p\left(T ; T^{\prime}\right) \phi\left(T^{\prime}\right) \\
& \leqslant\left(\max _{T^{\prime} \in \mathcal{T}_{\ell}} p\left(T ; T^{\prime}\right)\right) \sum_{T^{\prime} \in \mathcal{T}_{\ell}} \phi\left(T^{\prime}\right)=\left(\max _{T^{\prime} \in \mathcal{T}_{\ell}} p\left(T ; T^{\prime}\right)\right) \phi\left(1_{0}\right)=\left(\max _{T^{\prime} \in \mathcal{T}_{\ell}} p\left(T ; T^{\prime}\right)\right),
\end{aligned}
$$

for every $\ell \geqslant|T|$, since $\phi$ is linear and

$$
1_{0}=\sum_{T^{\prime} \in \mathcal{T}_{\ell}} T^{\prime}
$$

However, in general this bound is too weak to find extremal values. In what follows, we will present the semidefinite method, which builds up on this simple argument but can obtain much better bounds for Problem 13.

Let us start by defining some flag algebra notation that will help us.

Definition 15. Let $\sigma$ be a type. We define the semantic cone of type $\sigma$ as the set

$$
\mathcal{C}_{\mathrm{sem}}\left(\mathcal{F}^{\sigma}\right)=\left\{f \in \mathcal{A}^{\sigma}: \forall \phi \in \operatorname{Hom}^{+}\left(\mathcal{A}^{\sigma}, \mathbb{R}\right), \phi(f) \geqslant 0\right\},
$$

that is, the semantic cone is the set of all "positive" elements of $\mathcal{A}^{\sigma}$ with respect to positive homomorphisms.

We define also the ordinary cone of type $\sigma$ as the set

$$
\mathcal{C}\left(\mathcal{F}^{\sigma}\right)=\left\{\sum_{i=1}^{t} F_{i} \cdot f_{i}^{2}: t \in \mathbb{N} \wedge F_{1}, F_{2}, \ldots, F_{t} \in \mathcal{F}^{\sigma} \wedge f_{1}, f_{2}, \ldots, f_{t} \in \mathcal{A}^{\sigma}\right\} .
$$

Finally, we define the preorder relation $\leqslant_{\sigma}$ over $\mathcal{A}^{\sigma}$ through

$$
f \leqslant_{\sigma} g \Longleftrightarrow g-f \in \mathcal{C}_{\mathrm{sem}}\left(\mathcal{F}^{\sigma}\right) .
$$

Trivially we have $\mathcal{F}^{\sigma} \subset \mathcal{C}\left(\mathcal{F}^{\sigma}\right) \subset \mathcal{C}_{\text {sem }}\left(\mathcal{F}^{\sigma}\right)$.

The idea of the semidefinite method is to use elements of $\mathcal{C}_{\text {sem }}\left(\mathcal{F}^{0}\right)$ to compensate large $p\left(T ; T^{\prime}\right)$ in $(2)$ as follows. If $g \in \mathcal{C}_{\text {sem }}\left(\mathcal{F}^{0}\right)$, then

$$
T \leqslant_{0} T+g=\sum_{T^{\prime} \in \mathcal{T}_{\ell}}\left(p\left(T ; T^{\prime}\right)+p\left(g ; T^{\prime}\right)\right) T^{\prime}
$$




$$
\leqslant_{0}\left(\max _{T^{\prime} \in \mathcal{T}_{\ell}} p\left(T ; T^{\prime}\right)+p\left(g ; T^{\prime}\right)\right) \sum_{T^{\prime} \in \mathcal{T}_{\ell}} T^{\prime}=\left(\max _{T^{\prime} \in \mathcal{T}_{\ell}} p\left(T ; T^{\prime}\right)+p\left(g ; T^{\prime}\right)\right) 1_{0},
$$

where $\ell \in \mathbb{N}$ is large enough (so that we can write $g$ as a combination of tournaments of size smaller than $\ell$ ). Our hope in doing so is to be able to choose $g$ such that $p\left(g ; T^{\prime}\right)$ is negative when $p\left(T ; T^{\prime}\right)$ is large, but taking $p\left(g ; T^{\prime}\right)$ positive enough to ensure $g \in \mathcal{C}_{\text {sem }}\left(\mathcal{F}^{0}\right)$ when $p\left(T ; T^{\prime}\right)$ is small.

In this language, our problem is exactly to prove that $c 1_{0}-T \in \mathcal{C}_{\text {sem }}\left(\mathcal{F}^{0}\right)$ for a certain $c$. However, deciding whether an arbitrary $g$ is an element of $\mathcal{C}_{\text {sem }}\left(\mathcal{F}^{\sigma}\right)$ is hard. In fact, the flag algebra semidefinite method is part of what is called Cauchy-Schwarz calculus in flag algebras (see [28, Section $\S 6]$ ), which was proved to be incomplete for the theory of graphs in [20], that is, in the theory of graphs, there are elements of $\mathcal{C}_{\text {sem }}\left(\mathcal{F}^{0}\right)$ that cannot be proved to be in $\mathcal{C}_{\text {sem }}\left(\mathcal{F}^{0}\right)$ via Cauchy-Schwarz calculus (hence neither via semidefinite method).

We will now define the downward operator, which will help us in obtaining elements of $\mathcal{C}_{\text {sem }}\left(\mathcal{F}^{0}\right)$ from elements of $\mathcal{C}_{\text {sem }}\left(\mathcal{F}^{\sigma}\right)$.

Definition 16. Let $\sigma$ be a type of size $k$ and $F=(T, \theta)$ be a $\sigma$-flag. We denote the underlying tournament of $F$ by $\left.F\right|_{0}=T$ and we define the normalizing factor of $F$ (denoted $q_{\sigma}(F)$ ) through the following random experiment.

We pick uniformly at random an injective function $\boldsymbol{\theta}:[k] \rightarrow V\left(\left.F\right|_{0}\right)$ and let

$$
q_{\sigma}(F)=\mathbb{P}\left[\left(\left.F\right|_{0}, \boldsymbol{\theta}\right) \cong F\right] .
$$

We also define the downward operator $\llbracket \cdot \rrbracket_{\sigma}$ by letting

$$
\llbracket F \rrbracket_{\sigma}=\left.q_{\sigma}(F) F\right|_{0} \quad \in \mathcal{A}^{0}
$$

and extending it linearly to combinations of $\sigma$-flags.

Theorem 17 (Razborov [28, Theorems 2.5 and 3.1a]). The downward operator $\llbracket \cdot \rrbracket_{\sigma}$ is well-defined as an operator $\mathcal{A}^{\sigma} \rightarrow \mathcal{A}^{0}$ and we have

$$
\llbracket \mathcal{C}_{\mathrm{sem}}\left(\mathcal{F}^{\sigma}\right) \rrbracket_{\sigma} \subset \mathcal{C}_{\mathrm{sem}}\left(\mathcal{F}^{0}\right) .
$$

This theorem allows us to choose $g$ of $(3)$ in the easier set $\llbracket \mathcal{C}\left(\mathcal{F}^{\sigma}\right) \rrbracket_{\sigma}$ for some type $\sigma$. This reduces the problem to finding a positive semidefinite matrix in the following way.

Fix a type $\sigma$ of size $k$, a $\sigma$-flag $F^{\prime}$ and let $\widetilde{\ell}$ and $\ell$ be integers such that $k \leqslant \tilde{\ell}$ and $\left|F^{\prime}\right|+2 \widetilde{\ell}-2 k \leqslant \ell$.

If $v \in \mathbb{R}^{\mathcal{F}_{\tilde{\ell}}^{\sigma}}$ is a vector indexed by $\mathcal{F}_{\widetilde{\ell}}^{\sigma}$, then let $F(v)$ denote the element

$$
\sum_{F \in \mathcal{F}_{\bar{\ell}}^{\sigma}} v_{F} F \in \mathcal{A}^{\sigma}
$$

Analogously, if $Q$ is a matrix indexed by by $\mathcal{F}_{\tilde{\ell}}^{\sigma} \times \mathcal{F}_{\tilde{\ell}}^{\sigma}$, let $F(Q)$ denote the element

$$
\sum_{F_{1}, F_{2} \in \mathcal{F}_{\bar{\ell}}^{\sigma}} Q_{F_{1} F_{2}} F_{1} F_{2} \in \mathcal{A}^{\sigma}
$$


Note that if $Q$ is positive semidefinite $(Q \succeq 0)$, then by the Spectral Theorem there exist vectors $v_{1}, v_{2}, \ldots, v_{r} \in \mathbb{R}^{\mathcal{F}_{\tilde{\ell}}^{\sigma}}$ such that

$$
Q=\sum_{i=1}^{r} v_{i} v_{i}^{\top},
$$

which means that

$$
F(Q)=\sum_{i=1}^{r} F\left(v_{i}\right)^{2} .
$$

Hence we have $F^{\prime} \cdot F(Q) \in \mathcal{C}\left(\mathcal{F}^{\sigma}\right)$ and we can take $g$ in $(3)$ to be equal to

$$
\llbracket F^{\prime} \cdot F(Q) \rrbracket_{\sigma} .
$$

This yields the following semidefinite program

$\min y$

$$
\begin{array}{ll}
\text { s.t. } & p\left(T, T^{\prime}\right)+\sum_{F \in \mathcal{F}_{\ell}^{\sigma}} \sum_{F_{1}, F_{2} \in \mathcal{F}_{\bar{\ell}}^{\sigma}} Q_{F_{1} F_{2}} p\left(F^{\prime}, F_{i}, F_{j} ; F\right) p\left(\llbracket F \rrbracket_{\sigma} ; T^{\prime}\right) \leqslant y \quad \forall T^{\prime} \in \mathcal{T}_{\ell} ; \\
& Q \in \mathbb{R}^{\mathcal{F}_{\bar{\ell}}^{\sigma} \times \mathcal{F}_{\bar{\ell}}^{\sigma}} \text { is positive semidefinite; }
\end{array}
$$

whose solutions have values that are upper bounds to the value in Problem 13.

In fact, we can even take $g=\sum_{i=1}^{m} g_{i}$ in (3), where each $g_{i}$ is of the form

$$
\llbracket F_{i}^{\prime} \cdot F\left(Q_{i}\right) \rrbracket_{\sigma_{i}}
$$

for some type $\sigma_{i}$, some $\sigma_{i}$-flag $F_{i}^{\prime}$ and some positive semidefinite matrix $Q_{i}$ indexed by $\mathcal{F}_{\ell_{i}}^{\sigma_{i}} \times \mathcal{F}_{\ell_{i}}^{\sigma_{i}}$.

We state the resulting semidefinite program in the proposition below.

Proposition 18 ([29]). Let $T \in \mathcal{T}$ be a tournament, let $\sigma_{1}, \sigma_{2}, \ldots, \sigma_{m}$ be types of sizes $k_{1}, k_{2}, \ldots, k_{m}$ respectively and for each $t \in[m]$, let $F_{t}^{\prime} \in \mathcal{F}^{\sigma_{t}}$ be a $\sigma_{t}$-flag. Let also $\ell_{1}, \ell_{2}, \ldots, \ell_{m}, \ell$ be integers such that

$$
k_{t} \leqslant \ell_{t} ; \quad\left|F_{t}^{\prime}\right|+2 \ell_{t}-2 k_{t} \leqslant \ell ;
$$

for every $t \in[m]$ and such that $|T| \leqslant \ell$.

Under these circumstances, every value of every solution of the semidefinite program $\min y$

$$
\begin{array}{ll}
\text { s.t. } & p\left(T, T^{\prime}\right)+\sum_{t=1}^{m} \sum_{F \in \mathcal{F}_{\ell}^{\sigma_{t}}} \sum_{F_{1}, F_{2} \in \mathcal{F}_{\ell_{t}}^{\sigma_{t}}} Q_{F_{1} F_{2}}^{(t)} p\left(F_{t}^{\prime}, F_{i}, F_{j} ; F\right) p\left(\llbracket F \rrbracket_{\sigma_{t}} ; T^{\prime}\right) \leqslant y \quad \forall T^{\prime} \in \mathcal{T}_{\ell} ; \\
& Q^{(t)} \in \mathbb{R}^{\mathcal{F}_{\ell_{t}}^{\sigma_{t}} \times \mathcal{F}_{\ell_{t}}^{\sigma_{t}}} \text { is positive semidefinite } \quad \forall t \in[m] ;
\end{array}
$$

is an upper bound to the value in Problem 13, that is, if $V$ is the value of a solution of (4), then

$$
\max \left\{\phi(T): \phi \in \operatorname{Hom}^{+}\left(\mathcal{A}^{0}, \mathbb{R}\right)\right\} \leqslant V
$$


In this text, all instances of (4) will be with $F_{t}^{\prime}=1_{\sigma_{t}}$ for every $t \in[m]$. Furthermore, when we use Proposition 18 to give upper bounds to Problem 13, we will denote each of the $Q^{(t)}$ in (4) by $Q\left(T, \sigma_{t}\right)$ as a reminder of which problem we are solving and of what is the type involved. Moreover, for each $T^{\prime} \in \mathcal{T}_{\ell}$, we define

$$
\begin{aligned}
& c\left(Q\left(T, \sigma_{t}\right) ; T^{\prime}\right)= \\
& p\left(\llbracket F\left(Q\left(T, \sigma_{t}\right)\right) \rrbracket_{\sigma_{t}} ; T^{\prime}\right)=\sum_{F \in \mathcal{F}_{\ell}^{\sigma_{t}}} \sum_{F_{1}, F_{2} \in \mathcal{F}_{\ell_{t}}^{\sigma_{t}}} Q\left(T, \sigma_{t}\right)_{F_{1} F_{2}} p\left(F_{i}, F_{j} ; F\right) p\left(\llbracket F \rrbracket_{\sigma_{t}} ; T^{\prime}\right)
\end{aligned}
$$

and let

$$
c\left(T ; T^{\prime}\right)=\sum_{t=1}^{m} c\left(Q\left(T, \sigma_{t}\right) ; T^{\prime}\right)
$$

so that (4) becomes

$\min y$

$$
\begin{aligned}
& \text { s.t. } \quad p\left(T, T^{\prime}\right)+c\left(T ; T^{\prime}\right) \leqslant y \quad \forall T^{\prime} \in \mathcal{T}_{\ell} ; \\
& c\left(T ; T^{\prime}\right)=\sum_{t=1}^{m} c\left(Q\left(T, \sigma_{t}\right) ; T^{\prime}\right) \quad \forall T^{\prime} \in \mathcal{T}_{\ell} ; \\
& c\left(Q\left(T, \sigma_{t}\right) ; T^{\prime}\right)=\sum_{F \in \mathcal{F}_{\ell}^{\sigma_{t}}} \sum_{F_{1}, F_{2} \in \mathcal{F}_{\ell_{t}}^{\sigma_{t}}} Q\left(T, \sigma_{t}\right)_{F_{1} F_{2}} p\left(F_{i}, F_{j} ; F\right) p\left(\llbracket F \rrbracket_{\sigma_{t}} ; T^{\prime}\right) ; \\
& Q\left(T, \sigma_{t}\right) \in \mathbb{R}^{\mathcal{F}_{\ell_{t}}^{\sigma_{t}} \times \mathcal{F}_{\ell_{t}}^{\sigma_{t}}} \text { is positive semidefinite } \quad \forall t \in[m] .
\end{aligned}
$$

\subsection{Tournaments, types and flags used}

Throughout this text, we denote the transitive tournament of size $k$ by $\operatorname{Tr}_{k}$. We also denote (see Figure 6).

- the 3-cycle by $\vec{C}_{3}$;

- the only tournament of size 4 that has a 4 -cycle by $R_{4}$;

- the only tournament with outdegree sequence $(1,1,1,3)$ by $W_{4}$;

- the only tournament with outdegree sequence $(0,2,2,2)$ by $L_{4}$.

We will also use the notation of Figure 7 for the non isomorphic tournaments of size 5 . Furthermore, we define the following types (see Figure 6).

- The only type of size 1 is denoted by 1 ;

- The type of size 2 where the vertex with label 1 beats the vertex with label 2 is denoted $A$; 


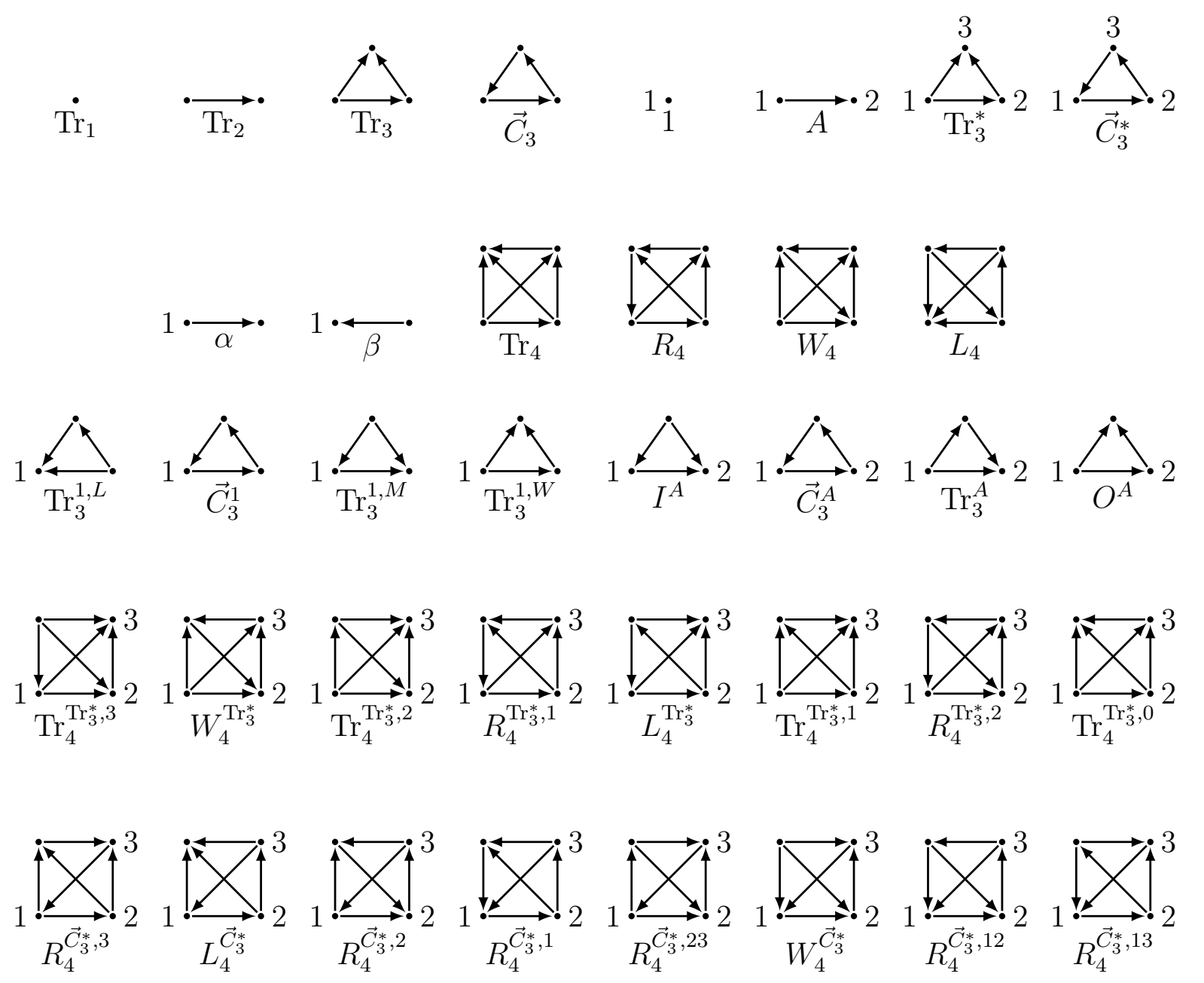

Figure 6: Types and flags of size at most 4 used.

- The type of size 3 isomorphic to $\operatorname{Tr}_{3}$ such that the winner has label 1 and the loser has label 3 is denoted $\operatorname{Tr}_{3}^{*}$;

- The type of size 3 isomorphic to $\vec{C}_{3}$ such that the vertex with label 1 beats the vertex with label 2 is denoted $\vec{C}_{3}^{*}$.

If $T$ is a tournament and $\sigma$ is a type such that there exists exactly one $\sigma$-flag $F$ such that $\left.F\right|_{0}=T$, then we denote such flag by $T^{\sigma}$. Note that this uniquely defines the following flags.

$$
\vec{C}_{3}^{1}, \vec{C}_{3}^{A}, W_{4}^{\operatorname{Tr}_{3}^{*}}, L_{4}^{\operatorname{Tr}_{3}^{*}}, W_{4}^{\vec{C}_{3}^{*}}, L_{4}^{\vec{C}_{3}^{*}}
$$

For the remaining flags, we use the notation of Figure 6. Let us only comment the reasoning behind our notation. 


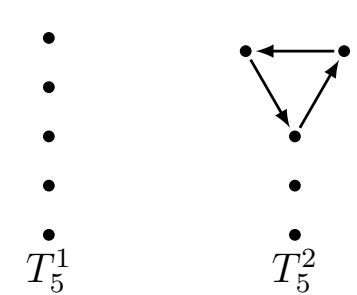

$\left(\operatorname{Tr}_{5}\right)$

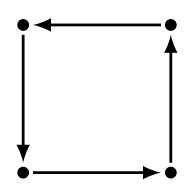

$\dot{T_{5}^{3}}$
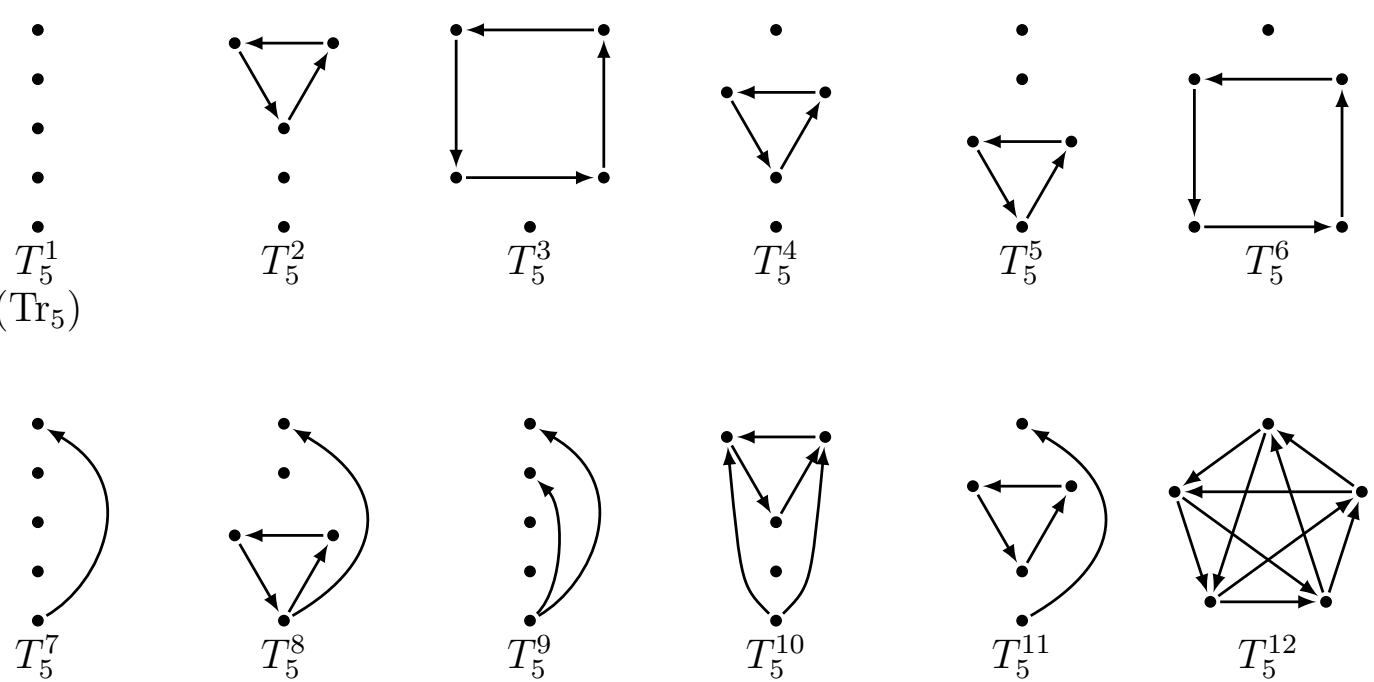

$\left(R_{5}\right)$

Figure 7: Tournaments of size 5. The arcs omitted are all oriented downward.

- The notation for the flags $O^{A}$ and $I^{A}$ are meant to be a mnemonic for common outneighbourhood and common inneighbourhood respectively;

- The flag $\operatorname{Tr}_{3}^{A}$ is not the only $A$-flag over $\operatorname{Tr}_{3}$, but this notation is nevertheless used since $\operatorname{Tr}_{3}^{A}$ is the only remaining $A$-flag over $\operatorname{Tr}_{3}$;

- The $\operatorname{Tr}_{3}^{*}$-flags over $\operatorname{Tr}_{4}$ and $R_{4}$ are uniquely determined by the outdegree $d$ of the unlabelled vertex and as such, we denote them accordingly by $\operatorname{Tr}_{4}^{\operatorname{Tr}_{3}^{*}, d}$ and $R_{4}^{\operatorname{Tr}_{3}^{*}, d}$;

- The $\vec{C}_{3}^{*}$-flags over $R_{4}$ are uniquely determined by the outneighbourhood of the unlabelled vertex and as such, we denote the accordingly by listing the vertices in the outneighbourhood of the unlabelled vertex in the superscript.

\section{Upper bounds}

In this section we prove the upper bounds in Theorems 2, 1 and 4. We use the semidefinite method of flag algebras as presented in Section 3.

Lemma 19. For every n-vertex tournament $T_{n}$,

$$
\lim _{n \rightarrow \infty} p\left(T_{5}^{12} ; T_{n}\right) \leqslant \frac{1}{16}
$$

Proof. In order to use the semidefinite method, we need to fix $\ell$, which is used to define set $\mathcal{T}_{\ell}$. Then we need to define $c\left(T ; T^{\prime}\right)$ for every $T^{\prime} \in \mathcal{T}_{\ell}$ as in (5). To define $c\left(T ; T^{\prime}\right)$, we choose how many types $m$ we will use and the types $\sigma_{t}$ we want to use. For each type $\sigma_{t}$, 
we choose an integer $\ell_{t}$ satisfying $\ell_{t} \leqslant\left(\ell+\left|\sigma_{t}\right|\right) / 2$ and a positive semidefinite $\left|\mathcal{F}_{\ell_{t}}^{\sigma_{t}}\right| \times\left|\mathcal{F}_{\ell_{t}}^{\sigma_{t}}\right|$ matrix $Q\left(T, \sigma_{t}\right)$.

Fix $m=3, \ell=5, \ell_{1}=3, \ell_{2}=\ell_{3}=4$ and let $\sigma_{1}=1, \sigma_{2}=\operatorname{Tr}_{3}^{*}$ and $\sigma_{3}=\vec{C}_{3}^{*}$ be types as defined in Section 3.3 (see Figure (6)).

Let $Q\left(T_{5}^{12}, 1\right), Q\left(T_{5}^{12}, \operatorname{Tr}_{3}^{*}\right)$ and $Q\left(T_{5}^{12}, \vec{C}_{3}^{*}\right)$ be the positive semidefinite matrices of orders $\mathcal{F}_{3}^{1} \times \mathcal{F}_{3}^{1}, \mathcal{F}_{4}^{\operatorname{Tr}_{3}^{*}} \times \mathcal{F}_{4}^{\operatorname{Tr}_{3}^{*}}$ and $\mathcal{F}_{4}^{\vec{C}_{3}^{*}} \times \mathcal{F}_{4}^{\vec{C}_{3}^{*}}$ respectively shown in Appendix A.1 (note that $\left|\mathcal{F}_{3}^{1}\right|=4$ and $\left.\left|\mathcal{F}_{4}^{\operatorname{Tr}_{3}^{*}}\right|=\left|\mathcal{F}_{4}^{\vec{C}_{3}^{*}}\right|=8\right)$.

To see that $Q\left(T_{5}^{12}, 1\right), Q\left(T_{5}^{12}, \operatorname{Tr}_{3}^{*}\right)$ and $Q\left(T_{5}^{12}, \vec{C}_{3}^{*}\right)$ are positive semidefinite, we analyse their characteristic polynomials $p_{Q\left(T_{5}^{12}, 1\right)}(x), p_{Q\left(T_{5}^{12}, \operatorname{Tr}_{3}^{*}\right)}(x)$ and $p_{Q\left(T_{5}^{12}, \vec{C}_{3}^{*}\right)}(x)$ shown in Appendix A.2. Since the only negative coefficients of these polynomials are all of odd order, it follows that all of their roots are non-negative, hence the matrices are positive semidefinite.

We then compute $p\left(T_{5}^{12}, T\right)$ and $c\left(Q\left(T_{5}^{12}, \sigma_{t}\right) ; T\right)$ for every $T \in \mathcal{T}_{5}$ (see Figure 7 ) and every $t \in[3]$.

Finally, by Proposition 18, we have

$$
\lim _{n \rightarrow \infty} p\left(T_{5}^{12} ; T_{n}\right) \leqslant \max _{T \in \mathcal{T}_{5}}\left\{p\left(T_{5}^{12} ; T\right)+c\left(T_{5}^{12} ; T\right)\right\}=\frac{1}{16}
$$

where $c\left(T_{5}^{12} ; T\right)=c\left(Q\left(T_{5}^{12}, 1\right) ; T\right)+c\left(Q\left(T_{5}^{12}, \operatorname{Tr}_{3}^{*}\right) ; T\right)+c\left(Q\left(T_{5}^{12}, \vec{C}_{3}^{*}\right) ; T\right)$ for every $T \in$ $\mathcal{T}_{5}$.

Remark 20. All of the matrices in Appendix A.1 were found with the aid of semidefinite programming solvers CSDP [5] and SDPA [33].

Furthermore, the solution provided by these solvers was rounded to an exact solution using the rounding method described by Baber [1].

The solutions presented here and the program utilized to produce the semidefinite program input, round the solutions provided by the solvers and check their correctness can be obtained in https://github.com/robertoparente/flag-algebra-program-package.

Finally, the characteristic polynomials in Appendix A.2 were found with the aid of the symbolic mathematics software Maxima [26].

The proofs of the upper bounds for $T_{5}^{7}, T_{5}^{8}, T_{5}^{9}$ and $T_{5}^{11}$ are very similar to the proof of Lemma 19. We choose how many types $m$ we will use and the types $\sigma_{i}$ we want to use. For each type $\sigma_{i}$, we choose an integer $\ell_{i}$ satisfying $\ell_{i} \leqslant\left(\ell+\left|\sigma_{i}\right|\right) / 2$ and find positive semidefinite matrices $Q_{i}=Q\left(T_{5}^{j}, \sigma_{i}\right)$.

The matrices and their characteristic polynomials are shown in Appendix A. As in the proof of Lemma 19, the matrices are easily seen to be positive semidefinite since the only negative coefficients of their characteristic polynomials are all of odd order.

For each $j \in\{7,8,9,11,12\}$, we then compute $c\left(T_{5}^{j} ; T\right)=\sum_{i=i}^{m} c\left(Q\left(T_{5}^{j}, \sigma_{i}\right) ; T\right)$ and $p\left(T_{5}^{j} ; T\right)$, for every $T \in \mathcal{T}_{\ell}$, and obtain the desired bounds according to the following tables. 


\begin{tabular}{|c|c|}
\hline$T_{5}^{7}$ & \\
\hline$m$ & 3 \\
$\sigma_{1}$ & 1 \\
$\sigma_{2}$ & $\operatorname{Tr}_{3}^{*}$ \\
$\sigma_{3}$ & $\vec{C}_{3}^{*}$ \\
$\ell$ & 5 \\
$\ell_{1}$ & 3 \\
$\ell_{2}$ & 4 \\
$\ell_{3}$ & 4 \\
$Q_{1}$ & $Q\left(T_{5}^{7}, 1\right)$ \\
$Q_{2}$ & $Q\left(T_{5}^{7}, \operatorname{Tr}_{3}^{*}\right)$ \\
$Q_{3}$ & $Q\left(T_{5}^{7}, \vec{C}_{3}^{*}\right)$ \\
\hline
\end{tabular}

\begin{tabular}{|c|c|}
\hline$T_{5}^{8}$ & \\
\hline$m$ & 4 \\
$\sigma_{1}$ & 1 \\
$\sigma_{2}$ & $A$ \\
$\sigma_{3}$ & $\operatorname{Tr}_{3}^{*}$ \\
$\sigma_{4}$ & $\vec{C}_{3}^{*}$ \\
$\ell$ & 5 \\
$\ell_{1}$ & 3 \\
$\ell_{2}$ & 3 \\
$\ell_{3}$ & 4 \\
$\ell_{4}$ & 4 \\
$Q_{1}$ & $Q\left(T_{5}^{8}, 1\right)$ \\
$Q_{2}$ & $Q\left(T_{5}^{8}, A\right)$ \\
$Q_{3}$ & $Q\left(T_{5}^{8}, \operatorname{Tr}_{3}^{*}\right)$ \\
$Q_{4}$ & $Q\left(T_{5}^{8}, \vec{C}_{3}^{*}\right)$ \\
\hline
\end{tabular}

\begin{tabular}{|c|c|c|c|}
\hline$T_{5}^{9}$ & & $T_{5}^{11}$ & \\
\hline$m$ & 2 & $m$ & 2 \\
$\sigma_{1}$ & 1 & $\sigma_{1}$ & 1 \\
$\sigma_{2}$ & $\vec{C}_{3}^{*}$ & $\sigma_{2}$ & $\vec{C}_{3}^{*}$ \\
$\ell$ & 5 & $\ell$ & 5 \\
$\ell_{1}$ & 3 & $\ell_{1}$ & 3 \\
$\ell_{2}$ & 4 & $\ell_{2}$ & 4 \\
$Q_{1}$ & $Q\left(T_{5}^{9}, 1\right)$ & $Q_{1}$ & $Q\left(T_{5}^{11}, 1\right)$ \\
$Q_{2}$ & $Q\left(T_{5}^{9}, \vec{C}_{3}^{*}\right)$ \\
$Q_{2}$ & $Q\left(T_{5}^{11}, \vec{C}_{3}^{*}\right)$ \\
\hline
\end{tabular}

\begin{tabular}{|c|c|}
\hline$T_{5}^{12}$ & \\
\hline$m$ & 3 \\
$\sigma_{1}$ & 1 \\
$\sigma_{2}$ & $\operatorname{Tr}_{3}^{*}$ \\
$\sigma_{3}$ & $\vec{C}_{3}^{*}$ \\
$\ell$ & 5 \\
$\ell_{1}$ & 3 \\
$\ell_{2}$ & 4 \\
$\ell_{3}$ & 4 \\
$Q_{1}$ & $Q\left(T_{5}^{12}, 1\right)$ \\
$Q_{2}$ & $Q\left(T_{5}^{12}, \mathrm{Tr}_{3}^{*}\right)$ \\
$Q_{3}$ & $Q\left(T_{5}^{12}, \vec{C}_{3}^{*}\right)$ \\
\hline
\end{tabular}

\section{Extracting more information from the semidefinite method}

In this section, we review some techniques in flag algebras to extract information about extremal homomorphisms of Problem 13 from a tight solution of the semidefinite program (4) (see also the more general version (5)). Again, we will work here only with the theory of tournaments, but these techniques can be used in a more general setting.

The first technique is used to prove that the tournaments $T^{\prime}$ corresponding to non-tight restrictions in (4) must have zero density in the extremal homomorphisms.

Proposition 21. Let $T \in \mathcal{T}$ be a tournament and let

$$
c=\max \left\{\phi(T): \phi \in \operatorname{Hom}^{+}\left(\mathcal{A}^{0}, \mathbb{R}\right)\right\} .
$$

If $\ell \geqslant|T|$ and $g \in \mathcal{C}_{\text {sem }}\left(\mathcal{F}^{0}\right)$ are such that

$$
\max \left\{p\left(T+g ; T^{\prime}\right): T^{\prime} \in \mathcal{T}_{\ell}\right\}=c,
$$

and $\phi \in \operatorname{Hom}^{+}\left(\mathcal{A}^{0}, \mathbb{R}\right)$ is extremal for $T$ (that is, if $\phi(T)=c$ ), then

$$
\phi\left(T^{\prime}\right)=0,
$$

for every $T^{\prime} \in \mathcal{T}_{\ell}$ such that $p\left(T+g ; T^{\prime}\right)<c$.

Proof. Recall the semidefinite method from Subsection 3.2. We know that

$$
\begin{aligned}
c & =\phi(T) \leqslant \phi(T+g)=\sum_{T^{\prime} \in \mathcal{T}_{\ell}} p\left(T+g ; T^{\prime}\right) \phi\left(T^{\prime}\right) \\
& \leqslant\left(\max _{T^{\prime} \in \mathcal{T}_{\ell}} p\left(T+g ; T^{\prime}\right)\right) \sum_{T^{\prime} \in \mathcal{T}_{\ell}} \phi\left(T^{\prime}\right)=c \phi\left(1_{0}\right)=c .
\end{aligned}
$$


Hence, we must have equality throughout. In particular, equality in the last inequality implies that

$$
\sum_{T^{\prime} \in \mathcal{T}_{\ell}} p\left(T+g ; T^{\prime}\right) \phi\left(T^{\prime}\right)=c \sum_{T^{\prime} \in \mathcal{T}_{\ell}} \phi\left(T^{\prime}\right)
$$

and since $\phi\left(T^{\prime}\right) \geqslant 0$ for every $T^{\prime} \in \mathcal{T}_{\ell}$, we have

$$
\phi\left(T^{\prime}\right)\left(c-p\left(T+g ; T^{\prime}\right)\right)=0
$$

for every $T^{\prime} \in \mathcal{T}_{\ell}$. Therefore, the result follows.

For the next technique, we will need the notion of a homomorphism extension, so we recall below the main theorem on the matter.

Theorem 22 (Razborov [28, Theorem 3.5]). If $\sigma$ is a type and $\phi \in \operatorname{Hom}^{+}\left(\mathcal{A}^{0}, \mathbb{R}\right)$ is a homomorphism such that $\phi(\sigma)>0$, then there exists a unique probability distribution of a random element $\boldsymbol{\phi}^{\sigma}$ of $\operatorname{Hom}^{+}\left(\mathcal{A}^{\sigma}, \mathbb{R}\right)$ (called homomorphism extension) satisfying

$$
\mathbb{E}\left[\phi^{\sigma}(f)\right]=\frac{\phi\left(\llbracket f \rrbracket_{\sigma}\right)}{\phi\left(\llbracket 1_{\sigma} \rrbracket_{\sigma}\right)},
$$

for every $f \in \mathcal{A}^{\sigma}$.

The next technique says that if the element $\llbracket F \cdot f^{2} \rrbracket_{\sigma}$ was used in a tight solution of (4), then we must have $\phi^{\sigma}(F \cdot f)=0$ almost surely for every extremal homomorphism $\phi \in$ $\operatorname{Hom}^{+}\left(\mathcal{A}^{0}, \mathbb{R}\right)$.

Proposition 23. With the definitions and notation of Proposition 18, let

$$
c=\max \left\{\phi(T): T \in \operatorname{Hom}^{+}\left(\mathcal{A}^{0}, \mathbb{R}\right)\right\},
$$

suppose that the optimum solution $\left(Q^{(t)}\right)_{t=1}^{m}$ of $(4)$ has value $c$ and write

$$
Q^{(t)}=\sum_{i=1}^{r_{t}} v_{i}^{(t)}\left(v_{i}^{(t)}\right)^{\top},
$$

for every $t \in[m]$.

Under these circumstances, if $\phi \in \operatorname{Hom}^{+}\left(\mathcal{A}^{0}, \mathbb{R}\right)$ is extremal for $T$, that is, if $\phi(T)=c$, then for every $t \in[m]$ with $\phi\left(\sigma_{t}\right)>0$ and every $i \in\left[r_{t}\right]$, we have

$$
\boldsymbol{\phi}^{\boldsymbol{\sigma}}\left(F_{t}^{\prime} \cdot F\left(v_{i}^{(t)}\right)\right)=0
$$

almost surely. 
Proof. Recall the semidefinite method from Subsection 3.2. We know that

$$
\begin{aligned}
c=\phi(T) & \leqslant \phi(T)+\sum_{i=1}^{m} \phi\left(\llbracket F_{t}^{\prime} \cdot F\left(Q^{(t)}\right) \rrbracket_{\sigma_{t}}\right) \\
& =\sum_{T^{\prime} \in \mathcal{T}_{\ell}}\left(p\left(T ; T^{\prime}\right)+\sum_{i=1}^{m} p\left(\llbracket F_{t}^{\prime} \cdot F\left(Q^{(t)}\right) \rrbracket_{\sigma_{t}} ; T^{\prime}\right)\right) \phi\left(T^{\prime}\right) \\
& \leqslant \max _{T^{\prime} \in \mathcal{T}_{\ell}}\left(p\left(T ; T^{\prime}\right)+\sum_{i=1}^{m} p\left(\llbracket F_{t}^{\prime} \cdot F\left(Q^{(t)}\right) \rrbracket_{\sigma_{t}} ; T^{\prime}\right)\right) \phi\left(1_{0}\right) \\
& =c .
\end{aligned}
$$

Hence, we must have equality throughout. In particular, equality in the first inequality implies that

$$
\sum_{i=1}^{m} \phi\left(\llbracket F_{t}^{\prime} \cdot F\left(Q^{(t)}\right) \rrbracket_{\sigma_{t}}\right)=0,
$$

and since $\llbracket F_{t}^{\prime} \cdot F\left(Q^{(t)}\right) \rrbracket_{\sigma_{t}} \in \mathcal{C}_{\text {sem }}\left(\mathcal{F}^{0}\right)$ for every $t \in[m]$, we get that

$$
\phi\left(\llbracket F_{t}^{\prime} \cdot F\left(Q^{(t)}\right) \rrbracket_{\sigma_{t}}\right)=0,
$$

for every $t \in[m]$.

Fix now $t \in[m]$ such that $\phi\left(\sigma_{t}\right)>0$ and recall that

$$
\llbracket F_{t}^{\prime} \cdot F\left(Q^{(t)}\right) \rrbracket_{\sigma_{t}}=\sum_{i=1}^{r_{t}} \llbracket F_{t}^{\prime} \cdot F\left(v_{i}^{(t)}\right)^{2} \rrbracket_{\sigma_{t}} .
$$

This along with (6) implies that

$$
\phi\left(\llbracket F_{t}^{\prime} \cdot F\left(v_{i}^{(t)}\right)^{2} \rrbracket_{\sigma_{t}}\right)=0 .
$$

From Theorem 22, we have

$$
\mathbb{E}\left[\phi^{\sigma_{t}}\left(F_{t}^{\prime} \cdot F\left(v_{i}^{(t)}\right)^{2}\right)\right]=0,
$$

and since this variable is (almost surely) non-negative, we get

$$
\phi^{\sigma_{t}}\left(F_{t}^{\prime} \cdot F\left(v_{i}^{(t)}\right)\right)=0
$$

almost surely, as desired.

\section{$6 \quad$ Uniqueness}

In this section, we will prove the uniqueness results. Namely, we will prove that a homomorphism $\phi \in \operatorname{Hom}^{+}\left(\mathcal{A}^{0}, \mathbb{R}\right)$ maximizes the density of $T_{5}^{8}$ if and only if $\phi$ is the quasirandom homomorphism $\phi_{\mathrm{qr}}$. We will also prove that $\phi \in \operatorname{Hom}^{+}\left(\mathcal{A}^{0}, \mathbb{R}\right)$ maximizes the density of $T_{5}^{7}$ or of $T_{5}^{12}\left(R_{5}\right)$ if and only if $\phi$ is the carousel homomorphism $\phi_{\mathrm{R}}$. Finally, we will also prove that $\phi \in \operatorname{Hom}^{+}\left(\mathcal{A}^{0}, \mathbb{R}\right)$ maximizes the density of $T_{5}^{9}$ or of $T_{5}^{11}$ if and only if $\phi$ is the limit of the sequence $\left(\vec{C}_{n}^{3}\right)_{n \in \mathbb{N}}$. 


\subsection{Quasi-random uniqueness}

First we recall the definition of the quasi-random homomorphism $\phi_{\mathrm{qr}} \in \operatorname{Hom}^{+}\left(\mathcal{A}^{0}, \mathbb{R}\right)$ as the almost sure limit of the sequence of random tournaments $\left(\boldsymbol{R}_{\boldsymbol{n}, \mathbf{1 / 2}}\right)_{n \in \mathbb{N}}$. Alternatively, the quasi-random homomorphism is defined by

$$
\phi_{\mathrm{qr}}(T)=\frac{\ell !}{|\operatorname{Aut}(T)| 2^{\left(\begin{array}{c}
\ell \\
2
\end{array}\right)},}
$$

for every tournament $T$ of size $\ell \in \mathbb{N}$, where $\operatorname{Aut}(T)$ denotes the group of automorphisms of the tournament $T$.

We also recall the equivalence of the following quasi-random properties in the lemma below.

Lemma 24 (Chung-Graham $\left[8\right.$, Theorem 1]). Let $\phi \in \operatorname{Hom}^{+}\left(\mathcal{A}^{0}, \mathbb{R}\right)$ be a homomorphism. The following are equivalent.

$$
\begin{aligned}
& P_{1}: \phi=\phi_{\mathrm{qr}} ; \\
& P_{4}: \phi^{\boldsymbol{A}}\left(O^{A}+I^{A}\right)=1 / 2 \text { a.s. }
\end{aligned}
$$

Remark 25. Although we will only use two quasi-random properties, let us mention that Chung and Graham proved equivalence of a total of 11 quasi-random properties $\left(P_{1}\right.$ to $\left.P_{11}\right)$.

We are now in condition of proving that the density of $T_{5}^{8}$ is maximized only by the quasi-random homomorphism.

Theorem 26. If $\phi \in \operatorname{Hom}^{+}\left(\mathcal{A}^{0}, \mathbb{R}\right)$ is a homomorphism, then

$$
\phi\left(T_{5}^{8}\right) \leqslant \frac{15}{128}
$$

with equality if and only if $\phi=\phi_{\mathrm{qr}}$.

Proof. By Lemma 8 and by Proposition 18 (see also Section 4), we know that

$$
\max \left\{\phi\left(T_{5}^{8}\right): \phi \in \operatorname{Hom}^{+}\left(\mathcal{A}^{0}, \mathbb{R}\right)\right\}=\frac{15}{128}=\phi_{\mathrm{qr}}\left(T_{5}^{8}\right) .
$$

Furthermore, we know that the matrices $Q\left(T_{5}^{8}, 1\right), Q\left(T_{5}^{8}, A\right), Q\left(T_{5}^{8}, \operatorname{Tr}_{3}^{*}\right)$ and $Q\left(T_{5}^{8}, \vec{C}_{3}^{*}\right)$ from the semidefinite method are an optimum solution with value 15/128.

Since

$$
Q\left(T_{5}^{8}, A\right)=\frac{99}{3200} v v^{\top}
$$

where $v=(1,-1,-1,1)$ (indexed by $\left(I^{A}, \vec{C}_{3}^{A}, \operatorname{Tr}_{3}^{A}, O^{A}\right)$ ), Proposition 23 implies that if $\phi \in \operatorname{Hom}^{+}\left(\mathcal{A}^{0}, \mathbb{R}\right)$ is such that $\phi\left(T_{5}^{8}\right)=15 / 128$, then

$$
\phi^{\boldsymbol{A}}(F(v))=\boldsymbol{\phi}^{\boldsymbol{A}}\left(I^{A}-\vec{C}_{3}^{A}-\operatorname{Tr}_{3}^{A}+O^{A}\right)=0 \text { a.s. }
$$


Since $\vec{C}_{3}^{A}+\operatorname{Tr}_{3}^{A}=1_{A}-O^{A}-I^{A}$, we get

$$
\phi^{A}\left(O^{A}+I^{A}\right)=\frac{1}{2} \text { a.s. }
$$

hence $\phi$ satisfies Property $P_{4}$ from Lemma 24. Therefore $\phi=\phi_{\mathrm{qr}}$.

\subsection{Quasi-carousel uniqueness}

First we recall the definition of the carousel homomorphism $\phi_{\mathrm{R}} \in \operatorname{Hom}^{+}\left(\mathcal{A}^{0}, \mathbb{R}\right)$ as the limit of the sequence $\left(R_{2 n+1}\right)_{n \in \mathbb{N}}$ of carousel tournaments. Analogously to quasi-random properties, the quasi-carousel properties [12] are equivalent properties over a homomorphism $\phi \in \operatorname{Hom}^{+}\left(\mathcal{A}^{0}, \mathbb{R}\right)$ that force $\phi=\phi_{\mathrm{R}}$. We recall two of the carousel properties below.

Lemma 27 ([12, Lemma 3.2]). Let $\phi \in \operatorname{Hom}^{+}\left(\mathcal{A}^{0}, \mathbb{R}\right)$ be a homomorphism. The following are equivalent.

$S_{1}: \phi=\phi_{\mathrm{R}} ;$

$S_{2}: \phi$ is balanced and locally transitive, that is, we have

$$
\boldsymbol{\phi}^{\mathbf{1}}(\alpha)=\boldsymbol{\phi}^{\mathbf{1}}(\beta) \text { a.s.; } \quad \phi\left(W_{4}+L_{4}\right)=0 .
$$

Furthermore, we will need an equivalence regarding balanced homomorphisms.

Lemma 28 (Chung-Graham $\left[8\right.$, Theorem 2]). Let $\phi \in \operatorname{Hom}^{+}\left(\mathcal{A}^{0}, \mathbb{R}\right)$ be a homomorphism. The following are equivalent.

$$
Q_{1}: \phi\left(\operatorname{Tr}_{3}\right)=3 / 4 \text { and } \phi\left(\vec{C}_{3}\right)=1 / 4 ;
$$

$Q_{4}: \phi$ is balanced, that is, we have $\boldsymbol{\phi}^{\mathbf{1}}(\alpha)=\boldsymbol{\phi}^{\mathbf{1}}(\beta)$ a.s.

Analogously to Theorem 26, uniqueness for the carousel homomorphism will follow from quasi-carousel Property $S_{2}$.

Theorem 29. If $\phi \in \operatorname{Hom}^{+}\left(\mathcal{A}^{0}, \mathbb{R}\right)$ is a homomorphism, then

$$
\phi\left(T_{5}^{7}\right) \leqslant \frac{5}{16}
$$

with equality if and only if $\phi=\phi_{\mathrm{R}}$.

Proof. By Lemma 6 and by Proposition 18 (see also Section 4), we know that

$$
\max \left\{\phi\left(T_{5}^{7}\right): \phi \in \operatorname{Hom}^{+}\left(\mathcal{A}^{0}, \mathbb{R}\right)\right\}=\frac{5}{16}=\phi_{\mathrm{R}}\left(T_{5}^{7}\right) .
$$

Our goal is to prove that every $\phi \in \operatorname{Hom}^{+}\left(\mathcal{A}^{0}, \mathbb{R}\right)$ such that $\phi\left(T_{5}^{7}\right)=5 / 16$ is balanced and locally transitive. 
To prove that such $\phi$ is balanced, we note that the matrices $Q\left(T_{5}^{7}, 1\right), Q\left(T_{5}^{7}, \operatorname{Tr}_{3}^{*}\right)$ and $Q\left(T_{5}^{7}, \vec{C}_{3}^{*}\right)$ from the semidefinite method are an optimum solution with value 5/16, and since

$$
Q\left(T_{5}^{7}, 1\right)=\frac{35}{48} v v^{\top}
$$

where $v=(1,-1,-1,1)$ (indexed by $\left(\operatorname{Tr}_{3}^{1, L}, \vec{C}_{3}^{1}, \operatorname{Tr}_{3}^{1, M}, \operatorname{Tr}_{3}^{1, W}\right)$ ), Proposition 23 implies that

$$
\boldsymbol{\phi}^{\mathbf{1}}(F(v))=\boldsymbol{\phi}^{\mathbf{1}}\left(\operatorname{Tr}_{3}^{1, L}-\vec{C}_{3}^{1}-\operatorname{Tr}_{3}^{1, M}+\operatorname{Tr}_{3}^{1, W}\right)=\boldsymbol{\phi}^{\mathbf{1}}\left((\alpha-\beta)^{2}\right)=0 \text { a.s. }
$$

Therefore $\boldsymbol{\phi}^{\mathbf{1}}(\alpha)=\boldsymbol{\phi}^{\mathbf{1}}(\beta)$ a.s., that is, the homomorphism $\phi$ is balanced.

To prove that $\phi$ is also locally transitive, we will use Proposition 21. Table 1 has the values of $p\left(T_{5}^{7}+g ; T^{\prime}\right)$ for $T^{\prime} \in \mathcal{T}_{5}$ and where

$$
\begin{array}{c|cccccccccccc}
g & =\llbracket F\left(Q\left(T_{5}^{7}, 1\right)\right) \rrbracket_{1}+\llbracket F\left(Q\left(T_{5}^{7}, \operatorname{Tr}_{3}^{*}\right)\right) \rrbracket_{\operatorname{Tr}_{3}^{*}}+\llbracket F\left(Q\left(T_{5}^{7}, \vec{C}_{3}^{*}\right)\right) \rrbracket_{\vec{C}_{3}^{*} \cdot} \\
T^{\prime} & T_{5}^{1} & T_{5}^{2} & T_{5}^{3} & T_{5}^{4} & T_{5}^{5} & T_{5}^{6} & T_{5}^{7} & T_{5}^{8} & T_{5}^{9} & T_{5}^{10} & T_{5}^{11} & T_{5}^{12} \\
\hline p\left(T_{5}^{7}+g ; T^{\prime}\right) & \frac{5}{16} & -\frac{7}{80} & \frac{11}{48} & -\frac{29}{240} & -\frac{7}{80} & \frac{11}{48} & \frac{5}{16} & -\frac{13}{48} & \frac{5}{16} & \frac{1}{16} & -\frac{109}{240} & \frac{5}{16}
\end{array}
$$

Table 1: Values $p\left(T_{5}^{7}+g ; T^{\prime}\right)$ for $T^{\prime} \in \mathcal{T}_{5}$ and where $g=\llbracket F\left(Q\left(T_{5}^{7}, 1\right)\right) \rrbracket_{1}+$ $\llbracket F\left(Q\left(T_{5}^{7}, \operatorname{Tr}_{3}^{*}\right)\right) \rrbracket_{\operatorname{Tr}_{3}^{*}}+\llbracket F\left(Q\left(T_{5}^{7}, \vec{C}_{3}^{*}\right)\right) \rrbracket_{\vec{C}_{3}^{*}}$.

Proposition 21 implies that if $\phi\left(T^{\prime}\right)>0$ for $T^{\prime} \in \mathcal{T}_{5}$, then $T^{\prime} \in\left\{T_{5}^{1}, T_{5}^{7}, T_{5}^{9}, T_{5}^{12}\right\}$, and since these four tournaments are the only locally transitive tournaments of size 5 (i.e., the only tournaments $T^{\prime} \in \mathcal{T}_{5}$ with $\left.p\left(W_{4}+L_{4} ; T^{\prime}\right)=0\right)$, we have $\phi\left(W_{4}+L_{4}\right)=0$, that is, the homomorphism $\phi$ is locally transitive.

Therefore $\phi$ satisfies quasi-carousel Property $S_{2}$, hence $\phi=\phi_{\mathrm{R}}$ by Lemma 27 .

Theorem 30. If $\phi \in \operatorname{Hom}^{+}\left(\mathcal{A}^{0}, \mathbb{R}\right)$ is a homomorphism, then

$$
\phi\left(T_{5}^{12}\right) \leqslant \frac{1}{16}
$$

with equality if and only if $\phi=\phi_{\mathrm{R}}$.

Proof. By Lemma 6 and by Proposition 18 (see also Section 4), we know that

$$
\max \left\{\phi\left(T_{5}^{12}\right): \phi \in \operatorname{Hom}^{+}\left(\mathcal{A}^{0}, \mathbb{R}\right)\right\}=\frac{1}{16}=\phi_{\mathrm{R}}\left(T_{5}^{12}\right) .
$$

Again, our goal is to prove that every $\phi \in \operatorname{Hom}^{+}\left(\mathcal{A}^{0}, \mathbb{R}\right)$ such that $\phi\left(T_{5}^{12}\right)=1 / 16$ is balanced and locally transitive. 
To prove that such $\phi$ is balanced, we note that the matrices $Q\left(T_{5}^{12}, 1\right), Q\left(T_{5}^{12}, \operatorname{Tr}_{3}^{*}\right)$ and $Q\left(T_{5}^{12}, \vec{C}_{3}^{*}\right)$ from the semidefinite method are an optimum solution with value $1 / 16$, and since

$$
Q\left(T_{5}^{12}, 1\right)=\frac{1}{16} v v^{\top}
$$

where $v=(1,-1,-1,1)$ (indexed by $\left.\left(\operatorname{Tr}_{3}^{1, L}, \vec{C}_{3}^{1}, \operatorname{Tr}_{3}^{1, M}, \operatorname{Tr}_{3}^{1, W}\right)\right)$, Proposition 23 implies that

$$
\boldsymbol{\phi}^{\mathbf{1}}(F(v))=\boldsymbol{\phi}^{\mathbf{1}}\left(\operatorname{Tr}_{3}^{1, L}-\vec{C}_{3}^{1}-\operatorname{Tr}_{3}^{1, M}+\operatorname{Tr}_{3}^{1, W}\right)=\boldsymbol{\phi}^{\mathbf{1}}\left((\alpha-\beta)^{2}\right)=0 \text { a.s. }
$$

Therefore $\phi^{\mathbf{1}}(\alpha)=\phi^{\mathbf{1}}(\beta)$ a.s., that is, the homomorphism $\phi$ is balanced.

To prove that $\phi$ is also locally transitive, we will use again Proposition 21. Table 2 has the values of $p\left(T_{5}^{12}+g ; T^{\prime}\right)$ for $T^{\prime} \in \mathcal{T}_{5}$ and where

$$
\begin{aligned}
& g=\llbracket F\left(Q\left(T_{5}^{12}, 1\right)\right) \rrbracket_{1}+\llbracket F\left(Q\left(T_{5}^{12}, \operatorname{Tr}_{3}^{*}\right)\right) \rrbracket_{\operatorname{Tr}_{3}^{*}}+\llbracket F\left(Q\left(T_{5}^{12}, \vec{C}_{3}^{*}\right)\right) \rrbracket_{\vec{C}_{3}^{*}} . \\
& \begin{array}{c|cccccccccccc}
T^{\prime} & T_{5}^{1} & T_{5}^{2} & T_{5}^{3} & T_{5}^{4} & T_{5}^{5} & T_{5}^{6} & T_{5}^{7} & T_{5}^{8} & T_{5}^{9} & T_{5}^{10} & T_{5}^{11} & T_{5}^{12} \\
\hline p\left(T_{5}^{12}+g ; T^{\prime}\right) & \frac{1}{16} & \frac{1}{80} & \frac{1}{16} & -\frac{3}{16} & \frac{1}{80} & \frac{1}{16} & \frac{1}{16} & \frac{1}{16} & \frac{1}{16} & \frac{1}{16} & -\frac{39}{80} & \frac{1}{16}
\end{array}
\end{aligned}
$$

Table 2: Values $p\left(T_{5}^{12}+g ; T^{\prime}\right)$ for $T^{\prime} \in \mathcal{T}_{5}$ and where $g=\llbracket F\left(Q\left(T_{5}^{12}, 1\right)\right) \rrbracket_{1}+$ $\llbracket F\left(Q\left(T_{5}^{12}, \operatorname{Tr}_{3}^{*}\right)\right) \rrbracket_{\operatorname{Tr}_{3}^{*}}+\llbracket F\left(Q\left(T_{5}^{12}, \vec{C}_{3}^{*}\right)\right) \rrbracket_{\vec{C}_{3}^{*}}$.

Proposition 21 implies that $\phi\left(T_{5}^{2}+T_{5}^{5}\right)=0$.

Now, since we have

$$
\llbracket\left(L_{4}^{\vec{C}_{3}^{*}}\right)^{2} \rrbracket_{\vec{C}_{3}^{*}}=\frac{1}{20} T_{5}^{2} ; \quad \llbracket\left(W_{4}^{\vec{C}_{3}^{*}}\right)^{2} \rrbracket_{\vec{C}_{3}^{*}}=\frac{1}{20} T_{5}^{5}
$$

and since $\phi$ is balanced, by Lemma 28 , we have $\phi\left(\vec{C}_{3}\right)=1 / 4$, hence

$$
\mathbb{E}\left[\phi^{\vec{C}_{3}^{*}}\left(W_{4}^{\vec{C}_{3}^{*}}\right)^{2}+\phi^{\vec{C}_{3}^{*}}\left(L_{4}^{\vec{C}_{3}^{*}}\right)^{2}\right]=\frac{1}{10} \cdot \frac{\phi\left(T_{5}^{2}+T_{5}^{5}\right)}{\phi\left(\vec{C}_{3}\right)}=0
$$

which implies that $\phi^{\vec{C}_{3}^{*}}\left(W_{4}^{\vec{C}_{3}^{*}}+L_{4}^{\vec{C}_{3}^{*}}\right)=0$ a.s.

This in turn implies that

$$
0=\mathbb{E}\left[\phi^{\vec{C}_{3}^{*}}\left(W_{4}^{\vec{C}_{3}^{*}}+L_{4}^{\vec{C}_{3}^{*}}\right)\right]=\frac{1}{4} \cdot \frac{\phi\left(W_{4}+L_{4}\right)}{\phi\left(\vec{C}_{3}\right)},
$$

hence $\phi\left(W_{4}+L_{4}\right)=0$, that is, the homomorphism $\phi$ is locally transitive.

Therefore $\phi$ satisfies quasi-carousel Property $S_{2}$, hence $\phi=\phi_{\mathrm{R}}$ by Lemma 27 . 


\subsection{Quasi-triangular uniqueness}

We start by defining a $\vec{C}_{3}$-decomposable tournament inductively, which intuitively are tournaments similar in structure to $\vec{C}_{n}^{3}$, but without requiring the "blow-up" to have parts as balanced as possible.

Definition 31. Define the sequence of sets $\left(\mathcal{B}_{n}\right)_{n \in \mathbb{N}}$ inductively as follows.

Let $\mathcal{B}_{0}=\mathcal{T}_{0}$ and $\mathcal{B}_{1}=\mathcal{T}_{1}$ and for $n \geqslant 2$, let $\mathcal{B}_{n} \subset \mathcal{T}_{n}$ be the set of all tournaments $T$ of size $n$ such that there exist sets $A, B$ and $C$ such that

i. The sets $A, B$ and $C$ are strictly contained in $V(T)$, that is, we have $A, B, C \subsetneq V(T)$;

ii. The sets $A, B$ and $C$ are pairwise disjoint;

iii. We have $V(T)=A \cup B \cup C$;

iv. We have $\left.T\right|_{A} \in \mathcal{B}_{|A|},\left.T\right|_{B} \in \mathcal{B}_{|B|}$ and $\left.T\right|_{C} \in \mathcal{B}_{|C|}$;

v. We have $A \times B, B \times C, C \times A \subset A(T)$.

Finally, we say that a tournament $T$ of size $n$ is $\vec{C}_{3}$-decomposable if $T \in \mathcal{B}_{n}$.

Remark 32. Note that items (i), (ii) and (iii) together say that $\{A, B, C\} \backslash\{\varnothing\}$ is a partition of $V(T)$ into either two or three sets.

Furthermore, note that item (ii) actually follows from item (v).

Finally, note that item (iv) is well-defined since $\max \{|A|,|B|,|C|\}<n$ (due to item (i)).

The next theorem provides a characterization of $\vec{C}_{3}$-decomposable theorems as the class of tournaments avoiding $T_{5}^{8}, T_{5}^{10}$ and $T_{5}^{12}$. We defer the proof of this theorem to Section 7.

Theorem 33. A tournament $T$ is $\vec{C}_{3}$-decomposable if and only if it has no copies of $T_{5}^{8}$, $T_{5}^{10}$ nor of $T_{5}^{12}$.

Motivated by the theorem above let us say that a homomorphism $\phi \in \operatorname{Hom}^{+}\left(\mathcal{A}^{0}, \mathbb{R}\right)$ in the theory of tournaments is $\vec{C}_{3}$-decomposable if $\phi\left(T_{5}^{8}+T_{5}^{10}+T_{5}^{12}\right)=0$.

Note that the fact that a sequence of tournaments $\left(T_{n}\right)_{n \in \mathbb{N}}$ converges to a $\vec{C}_{3}$-decomposable homomorphism does not imply that any of the tournaments is $\vec{C}_{3}$-decomposable. Rather, it only implies that the densities of the tournaments $T_{5}^{8}, T_{5}^{10}$ and $T_{5}^{12}$ in $T_{n}$ go to zero as $n$ goes to infinity.

We now define the notion of a $k$-equally $\vec{C}_{3}$-decomposable tournament inductively.

Definition 34. A tournament $T$ is 0-equally $\vec{C}_{3}$-decomposable if it is $\vec{C}_{3}$-decomposable.

For $k>0$, a tournament $T$ is $k$-equally $\vec{C}_{3}$-decomposable if either $|T| \leqslant 1$ or there exists $(A, B, C)$ as in Definition 31 satisfying also the following properties. 
a. We have

$$
\max \{|A|,|B|,|C|\}-\min \{|A|,|B|,|C|\} \leqslant 1
$$

b. The tournaments $\left.T\right|_{A},\left.T\right|_{B}$ and $\left.T\right|_{C}$ are $(k-1)$-equally $\vec{C}_{3}$-decomposable.

Trivially, every $k$-equally $\vec{C}_{3}$-decomposable tournament is also $(k-1)$-equally $\vec{C}_{3^{-}}$ decomposable.

Note also that if $n \leqslant 3^{k}$, then the only $k$-equally $\vec{C}_{3}$-decomposable tournament of size $n$ is $\vec{C}_{n}^{3}$. We claim now that the sequence $\left(\vec{C}_{n}^{3}\right)_{n \in \mathbb{N}}$ is convergent, but we defer the proof of this claim. We will call the limit of this sequence the triangular homomorphism and denote it by $\phi_{\vec{C}_{3}}$.

The next theorem states the equivalence of what we could call quasi-triangular properties. The equivalence of Properties $L_{1}, L_{2}$ and $L_{3}$ imply that $\phi_{\vec{C}_{3}}$ is the only homomorphism that maximizes the density of $T_{5}^{9}$ and is the only homomorphism that maximizes the density of $T_{5}^{11}$.

Theorem 35. If $\phi \in \operatorname{Hom}^{+}\left(\mathcal{A}^{0}, \mathbb{R}\right)$ is a homomorphism in the theory of tournaments, then the following are equivalent.

$L_{1}: \phi=\phi_{\vec{C}_{3}}$

$L_{2}: \phi$ maximizes the density of $T_{5}^{9}$, that is, we have

$$
\phi\left(T_{5}^{9}\right)=\max \left\{\psi\left(T_{5}^{9}\right): \psi \in \operatorname{Hom}^{+}\left(\mathcal{A}^{0}, \mathbb{R}\right)\right\} ;
$$

$L_{3}: \phi$ maximizes the density of $T_{5}^{11}$, that is, we have

$$
\phi\left(T_{5}^{11}\right)=\max \left\{\psi\left(T_{5}^{11}\right): \psi \in \operatorname{Hom}^{+}\left(\mathcal{A}^{0}, \mathbb{R}\right)\right\}
$$

$L_{4}: \phi$ is balanced and $\vec{C}_{3}$-decomposable, that is, we have

$$
\phi^{\mathbf{1}}(\alpha)=\phi^{\mathbf{1}}(\beta) \quad \text { a.s.; } \quad \phi\left(T_{5}^{8}+T_{5}^{10}+T_{5}^{12}\right)=0
$$

$L_{5}:$ For every $k \in \mathbb{N}$, there exists a sequence $\left(T_{n}^{(k)}\right)_{n \in \mathbb{N}}$ of $k$-equally $\vec{C}_{3}$-decomposable tournaments that converges to $\phi$.

We will prove Theorem 35 through a series of lemmas. We have already proved in Sections 2 and 4 that $L_{1} \Longrightarrow L_{2} \wedge L_{3}$.

The next two lemmas follow from the techniques presented in Section 5 .

Lemma 36. We have $L_{2} \Longrightarrow L_{4}$. 
Proof. By Lemma 7 and by Proposition 18 (see also Section 4), we know that

$$
\max \left\{\psi\left(T_{5}^{9}\right): \psi \in \operatorname{Hom}^{+}\left(\mathcal{A}^{0}, \mathbb{R}\right)\right\}=\frac{3}{8},
$$

and that the matrices $Q\left(T_{5}^{9}, 1\right)$ and $Q\left(T_{5}^{9}, \vec{C}_{3}^{*}\right)$ from the semidefinite method are an optimum solution with value $3 / 8$.

Let then $\phi \in \operatorname{Hom}^{+}\left(\mathcal{A}^{0}, \mathbb{R}\right)$ be a homomorphism that maximizes the density of $T_{5}^{9}$.

Let us prove that $\phi$ is $\vec{C}_{3}$-decomposable. To do this, we will use Proposition 21. Table 3 has the values of $p\left(T_{5}^{9}+g ; T^{\prime}\right)$ for $T^{\prime} \in \mathcal{T}_{5}$ and where

$$
\begin{array}{c|cccccccccccc}
\multicolumn{10}{c}{g=\llbracket F\left(Q\left(T_{5}^{9}, 1\right)\right) \rrbracket_{1}+\llbracket F\left(Q\left(T_{5}^{9}, \vec{C}_{3}^{*}\right)\right) \rrbracket_{\vec{C}_{3}^{*}} .} \\
T^{\prime} & T_{5}^{1} & T_{5}^{2} & T_{5}^{3} & T_{5}^{4} & T_{5}^{5} & T_{5}^{6} & T_{5}^{7} & T_{5}^{8} & T_{5}^{9} & T_{5}^{10} & T_{5}^{11} & T_{5}^{12} \\
\hline p\left(T_{5}^{9}+g ; T^{\prime}\right) & \frac{3}{8} & \frac{3}{8} & \frac{3}{8} & \frac{3}{8} & \frac{3}{8} & \frac{3}{8} & \frac{3}{8} & -\frac{289}{200} & \frac{3}{8} & \frac{3}{200} & \frac{3}{8} & \frac{11}{40}
\end{array}
$$

Table 3: Values $p\left(T_{5}^{9}+g ; T^{\prime}\right)$ for $T^{\prime} \in \mathcal{T}_{5}$ and where $g=\llbracket F\left(Q\left(T_{5}^{9}, 1\right)\right) \rrbracket_{1}+\llbracket F\left(Q\left(T_{5}^{9}, \vec{C}_{3}^{*}\right)\right) \rrbracket_{\vec{C}_{3}^{*}}$.

Proposition 21 implies that $\phi\left(T_{5}^{8}+T_{5}^{10}+T_{5}^{12}\right)=0$, that is, the homomorphism $\phi$ is $\vec{C}_{3}$-decomposable.

It remains only to prove that $\phi$ is balanced.

We first note that the matrix $Q\left(T_{5}^{9}, 1\right)$ has eigenvectors

$$
\begin{aligned}
& v_{1}=\left(1, \frac{-16+\sqrt{179}}{7}, \frac{2-\sqrt{179}}{7}, 1\right) \text {; } \\
& v_{2}=\left(\begin{array}{llll}
1, & \frac{-16-\sqrt{179}}{7}, & \frac{2+\sqrt{179}}{7}, & 1
\end{array}\right)
\end{aligned}
$$

(indexed by $\left(\operatorname{Tr}_{3}^{1, L}, \vec{C}_{3}^{1}, \operatorname{Tr}_{3}^{1, M}, \operatorname{Tr}_{3}^{1, W}\right)$ ) with the eigenvalues $12(16+\sqrt{179})$ and $12(16-$ $\sqrt{179})$ respectively.

By Proposition 23, we know that $\phi^{\mathbf{1}}\left(F\left(v_{1}\right)\right)=\phi^{\mathbf{1}}\left(F\left(v_{2}\right)\right)=0$ a.s. This implies that $0=\mathbb{E}\left[\phi^{\mathbf{1}}\left(F\left(v_{1}\right)-F\left(v_{2}\right)\right)\right]=\frac{2 \sqrt{179}}{7} \mathbb{E}\left[\phi^{\mathbf{1}}\left(\vec{C}_{3}^{1}-\operatorname{Tr}_{3}^{1, M}\right)\right]=\frac{2 \sqrt{179}}{7}\left(\phi\left(\vec{C}_{3}\right)-\frac{1}{3} \phi\left(\operatorname{Tr}_{3}\right)\right)$.

Hence $\phi\left(\vec{C}_{3}\right)=1 / 4$ (since $\left.\vec{C}_{3}+\operatorname{Tr}_{3}=1_{0}\right)$, which by Lemma 28 , implies that $\phi$ is balanced.

Therefore $\phi$ satisfies $L_{4}$.

Lemma 37. We have $L_{3} \Longrightarrow L_{4}$. 
Proof. By Lemma 7 and by Proposition 18 (see also Section 4), we know that

$$
\max \left\{\psi\left(T_{5}^{11}\right): \psi \in \operatorname{Hom}^{+}\left(\mathcal{A}^{0}, \mathbb{R}\right)\right\}=\frac{1}{16},
$$

and that the matrices $Q\left(T_{5}^{11}, 1\right)$ and $Q\left(T_{5}^{11}, \vec{C}_{3}^{*}\right)$ from the semidefinite method are an optimum solution with value $1 / 16$.

Let then $\phi \in \operatorname{Hom}^{+}\left(\mathcal{A}^{0}, \mathbb{R}\right)$ be a homomorphism that maximizes the density of $T_{5}^{11}$.

Since

$$
Q\left(T_{5}^{11}, 1\right)=\frac{5}{16} v v^{\top}
$$

where $v=(1,-1,-1,1)$ (indexed by $\left(\operatorname{Tr}_{3}^{1, L}, \vec{C}_{3}^{1}, \operatorname{Tr}_{3}^{1, M}, \operatorname{Tr}_{3}^{1, W}\right)$ ), Proposition 23 implies that

$$
\boldsymbol{\phi}^{\mathbf{1}}(F(v))=\boldsymbol{\phi}^{\mathbf{1}}\left(\operatorname{Tr}_{3}^{1, L}-\vec{C}_{3}^{1}-\operatorname{Tr}_{3}^{1, M}+\operatorname{Tr}_{3}^{1, W}\right)=\boldsymbol{\phi}^{\mathbf{1}}\left((\alpha-\beta)^{2}\right) 0 \text { a.s. }
$$

Therefore $\phi^{\mathbf{1}}(\alpha)=\phi^{\mathbf{1}}(\beta)$ a.s., that is, the homomorphism $\phi$ is balanced.

It remains only to prove that $\phi$ is $\vec{C}_{3}$-decomposable. To do this, we will use Proposition 21. Table 4 has the values of $p\left(T_{5}^{11}+g ; T^{\prime}\right)$ for $T^{\prime} \in \mathcal{T}_{5}$ and where

$$
\begin{array}{c|ccccccccccccc}
g & =\llbracket F\left(Q\left(T_{5}^{11}, 1\right)\right) \rrbracket_{1}+\llbracket F\left(Q\left(T_{5}^{11}, \vec{C}_{3}^{*}\right)\right) \rrbracket_{\vec{C}_{3}^{*}} \cdot \\
T^{\prime} & T_{5}^{1} & T_{5}^{2} & T_{5}^{3} & T_{5}^{4} & T_{5}^{5} & T_{5}^{6} & T_{5}^{7} & T_{5}^{8} & T_{5}^{9} & T_{5}^{10} & T_{5}^{11} & T_{5}^{12} \\
\hline p\left(T_{5}^{11}+g ; T^{\prime}\right) & \frac{1}{16} & \frac{1}{16} & \frac{1}{16} & \frac{1}{16} & \frac{1}{16} & \frac{1}{16} & \frac{1}{16} & -\frac{3}{400} & \frac{1}{16} & -\frac{23}{400} & \frac{1}{16} & \frac{1}{80}
\end{array}
$$

Table 4: Values $p\left(T_{5}^{11}+g ; T^{\prime}\right)$ for $T^{\prime} \in \mathcal{T}_{5}$ and where $g=\llbracket F\left(Q\left(T_{5}^{11}, 1\right)\right) \rrbracket_{1}+$ $\llbracket F\left(Q\left(T_{5}^{11}, \vec{C}_{3}^{*}\right)\right) \rrbracket_{\vec{C}_{3}^{*}}$.

Proposition 21 implies that $\phi\left(T_{5}^{8}+T_{5}^{10}+T_{5}^{12}\right)=0$, that is, the homomorphism $\phi$ is $\vec{C}_{3}$-decomposable.

Therefore $\phi$ satisfies $L_{4}$.

For the next two implications, we will need to use the notion of a $\vec{C}_{3}$-decomposition of a $\vec{C}_{3}$-decomposable tournament. To make it precise, let us first fix some notation. Let

$$
\Sigma^{*}=\left\{\left(\sigma_{i}\right)_{i=1}^{k}: k \in \mathbb{N} \wedge \forall i \in[k], \sigma_{i} \in[3]\right\}
$$

denote the set of all finite sequences of elements in $[3]=\{1,2,3\}$ (and let us denote the empty sequence by $\epsilon$ ).

As usual, we will denote by $\sigma \tau$ the sequence obtained by concatenating $\tau \in \Sigma^{*}$ to the end of $\sigma \in \Sigma^{*}$ and we will denote the length of a sequence $\sigma \in \Sigma^{*}$ by $|\sigma|$. 
Definition 38. Let $T$ be a $\vec{C}_{3}$-decomposable tournament. A $\vec{C}_{3}$-decomposition of $T$ is a family of sets $A=\left(A_{\sigma}\right)_{\sigma \in \Sigma^{*}}$ indexed by $\Sigma^{*}$ such that

i. We have $A_{\epsilon}=V(T)$;

ii. For every $\sigma \in \Sigma^{*}$ such that $\left|A_{\sigma}\right| \geqslant 2$, the triple $\left(A_{\sigma 1}, A_{\sigma 2}, A_{\sigma 3}\right)$ satisfies the items in Definition 31 for $\left.T\right|_{A_{\sigma}}$;

iii. For every $\sigma \in \Sigma^{*}$ such that $\left|A_{\sigma}\right| \leqslant 1$, the sets $A_{\sigma 1}, A_{\sigma 2}$ and $A_{\sigma 3}$ are pairwise disjoint and $A_{\sigma 1} \cup A_{\sigma 2} \cup A_{\sigma 3}=A_{\sigma}$.

For every $k \in \mathbb{N}$, the $k$-th level of the $\vec{C}_{3}$-decomposition $A$ is the family of sets $A_{\sigma}$ such that $|\sigma|=k$. The skewness of the $k$-th level of $A$ (denoted $\Delta_{k}(A)$ ) is defined as

$$
\Delta_{k}(A)=\max \left\{\left|A_{\sigma}\right|: \sigma \in \Sigma^{*} \wedge|\sigma|=k\right\}-\min \left\{\left|A_{\sigma}\right|: \sigma \in \Sigma^{*} \wedge|\sigma|=k\right\} .
$$

Note that a tournament is $k$-equally $\vec{C}_{3}$-decomposable if and only if it has a $\vec{C}_{3^{-}}$ decomposition $A=\left(A_{\sigma}\right)_{\sigma \in \Sigma^{*}}$ such that $\Delta_{\ell}(A) \leqslant 1$ for every $\ell \leqslant k$.

Let us now define some notation on tournaments.

Definition 39. Let $T$ be a tournament and $A \subset V(T)$. We define

$$
\begin{aligned}
& N^{+}(A)=\{v \in V(T): \forall a \in A, a v \in A(T)\} \\
& N^{-}(A)=\{v \in V(T): \forall a \in A, v a \in A(T)\} .
\end{aligned}
$$

Note that $N^{+}(A) \cup N^{-}(A)$ is always a subset of $V(T) \backslash A$ and may be a proper subset. We now prove two basic facts about tournaments.

Lemma 40. If $\left(T_{n}\right)_{n \in \mathbb{N}}$ is a sequence of tournaments with $\lim _{n \rightarrow \infty}\left|T_{n}\right|=\infty$ and $c \geqslant 1 / 2$ is a constant such that all but $o\left(\left|T_{n}\right|\right)$ vertices of $T_{n}$ have indegree greater than $(c+o(1))\left|T_{n}\right|$, then $c=1 / 2$.

Proof. Let $\left(T_{n}^{\prime}\right)_{n \in \mathbb{N}}$ be a convergent subsequence of $\left(T_{n}\right)_{n \in \mathbb{N}}$ and let $\phi \in \operatorname{Hom}^{+}\left(\mathcal{A}^{0}, \mathbb{R}\right)$ be its limit and note that $\phi^{\mathbf{1}}(\beta) \geqslant c$ a.s.

Since $\mathbb{E}\left[\phi^{\mathbf{1}}(\beta)\right]=1 / 2$, we get $c \leqslant 1 / 2$.

Lemma 41. Let $\left(T_{n}\right)_{n \in \mathbb{N}}$ be a sequence of tournaments converging to a balanced homomorphism $\phi$ and for every $n \in \mathbb{N}$, let $A_{n} \subset V\left(T_{n}\right)$ be such that $\left|A_{n}\right|=\Omega\left(\left|T_{n}\right|\right)$.

Under these circumstances, if $N^{+}\left(A_{n}\right) \cup N^{-}\left(A_{n}\right)=V\left(T_{n}\right) \backslash A_{n}$ for every $n \in \mathbb{N}$, then

$$
\left|N^{+}\left(A_{n}\right)\right|-\left|N^{-}\left(A_{n}\right)\right|=o\left(\left|T_{n}\right|\right) .
$$

Proof. Suppose not. This means that by passing to a subsequence and possibly flipping all arcs, we may suppose that there exists $\epsilon>0$ such that

$$
\left|N^{+}\left(A_{n}\right)\right|-\left|N^{-}\left(A_{n}\right)\right| \geqslant \epsilon\left|T_{n}\right|,
$$

for every $n \in \mathbb{N}$. 
Note that if $v \in A_{n}$, then we have

$$
\begin{aligned}
\left|T_{n}\right|-2 d^{-}(v) & \geqslant d^{+}(v)-d^{-}(v)+1 \\
& =\left|N^{+}\left(A_{n}\right)\right|+d_{A_{n}}^{+}(v)-\left|N^{-}\left(A_{n}\right)\right|-d_{A_{n}}^{-}(v)+1 \\
& \geqslant \epsilon\left|T_{n}\right|+d_{A_{n}}^{+}(v)-d_{A_{n}}^{-}(v)+1 \\
& =\epsilon\left|T_{n}\right|+\left|A_{n}\right|-2 d_{A_{n}}^{-}(v) \\
& \geqslant(1+\epsilon)\left|A_{n}\right|-2 d_{A_{n}}^{-}(v),
\end{aligned}
$$

where $d_{A}^{+}(v)=\left|N^{+}(v) \cap A\right|$ and $d_{A}^{-}(v)=\left|N^{-}(v) \cap A\right|$.

Since $\phi$ is balanced, we know that all but $o\left(\left|T_{n}\right|\right)$ vertices of $A_{n}$ have outdegree $(1 / 2+$ $o(1))\left|T_{n}\right|$, hence, since $\left|A_{n}\right|=\Omega\left(\left|T_{n}\right|\right)$, if $v$ is one such vertex, we have

$$
d_{A_{n}}^{-}(v) \geqslant\left(\frac{1+\epsilon}{2}+o(1)\right)\left|A_{n}\right|
$$

But this contradicts Lemma 40 for the sequence $\left(\left.T_{n}\right|_{A_{n}}\right)_{n \in \mathbb{N}}$.

The next technical lemma says that if a sequence of $\vec{C}_{3}$-decomposable tournaments converges to a balanced homomorphism, then we may suppose that at least two of $A_{1}^{(n)}$, $A_{2}^{(n)}$ or $A_{3}^{(n)}$ have non-negligible size. We defer the proof of this lemma to Section 8.

Lemma 42. If $\left(T_{n}\right)_{n \in \mathbb{N}}$ is a sequence of $\vec{C}_{3}$-decomposable tournaments that converges to a balanced homomorphism $\phi \in \operatorname{Hom}^{+}\left(\mathcal{A}^{0}, \mathbb{R}\right)$, then there exists a sequence $\left(T_{n}^{\prime}\right)_{n \in \mathbb{N}}$ of $\vec{C}_{3}$ decomposable tournaments and for every $n \in \mathbb{N}$ a $\vec{C}_{3}$-decomposition $\left(A_{\sigma}^{(n)}\right)_{\sigma \in \Sigma^{*}}$ of $T_{n}^{\prime}$ such that

- There exists a subsequence $\left(T_{k_{n}}\right)_{n \in \mathbb{N}}$ of $\left(T_{n}\right)_{n \in \mathbb{N}}$ such that the tournament $T_{n}^{\prime}$ can be obtained from $T_{k_{n}}$ by flipping o $\left(\left|T_{k_{n}}\right|^{2}\right)$ arcs (hence $\left(T_{n}^{\prime}\right)_{n \in \mathbb{N}}$ also converges to $\left.\phi\right)$;

- We have $\left|A_{1}^{(n)}\right|=\Omega\left(\left|T_{n}^{\prime}\right|\right)$ and $\left|A_{2}^{(n)}\right|=\Omega\left(\left|T_{n}^{\prime}\right|\right)$.

Lemma 43. We have $L_{4} \Longrightarrow L_{5}$.

Proof. Suppose that $\phi$ is balanced and $\vec{C}_{3}$-decomposable. Let $T_{\vec{C}_{3}}$ be the universal theory of $\vec{C}_{3}$-decomposable tournaments, that is, the theory of tournaments that have no copy of $T_{5}^{8}, T_{5}^{10}$ nor of $T_{5}^{12}$ and note that $\phi$ can also be thought of as an element of $\operatorname{Hom}^{+}\left(\mathcal{A}^{0}\left[T_{\vec{C}_{3}}\right], \mathbb{R}\right)$. This means that there exists a sequence $\left(T_{n}^{(0)}\right)_{n \in \mathbb{N}}$ of $\vec{C}_{3}$-decomposable tournaments that converges to $\phi$ (which is, by definition, a sequence of 0-equally $\vec{C}_{3}$-decomposable tournaments). Furthermore, we may also suppose without loss of generality that $\left|T_{n}^{(0)}\right|$ is a power of 3 for every $n \in \mathbb{N}$.

Let us now construct by induction in $k$ the sequences $\left(T_{n}^{(k)}\right)_{n \in \mathbb{N}}$ of $k$-equally $\vec{C}_{3^{-}}$ decomposable tournaments converging to $\phi$ and preserving the property that $\left|T_{n}^{(k)}\right|$ is a power of 3 for every $n \in \mathbb{N}$. 
Suppose $k>0$ and that we have already constructed $\left(T_{n}^{(k-1)}\right)_{n \in \mathbb{N}}$. Applying Lemma 42 a total of $3^{k-1}$ times to the tournaments induced by the $(k-1)$-th level of the $\vec{C}_{3^{-}}$ decompositions of the $T_{n}^{(k-1)}$, we know that there exists a sequence $\left(T_{n}^{\prime}\right)_{n \in \mathbb{N}}$ of $\vec{C}_{3}$-decomposable tournaments and for every $n \in \mathbb{N}$ there is a $\vec{C}_{3}$-decomposition $A^{(n)}=\left(A_{\sigma}^{(n)}\right)_{\sigma \in \Sigma^{*}}$ of $T_{n}^{\prime}$ such that

- For every $t \leqslant k-1$, we have $\Delta_{t}\left(A^{(n)}\right)=0$;

- There exists a subsequence $\left(T_{m_{n}}^{(k-1)}\right)_{n \in \mathbb{N}}$ of $\left(T_{n}^{(k-1)}\right)_{n \in \mathbb{N}}$ such that the tournament $T_{n}^{\prime}$ can be obtained from $T_{m_{n}}^{(k-1)}$ by flipping $o\left(\left|T_{m_{n}}^{(k-1)}\right|^{2}\right)$ arcs, all completely contained within one of the sets $A_{\sigma}^{(n)}$ for some $\sigma \in \Sigma^{*}$ with $|\sigma|=k-1$;

- For every $\sigma \in \Sigma^{*}$ with $|\sigma|=k-1$, we have

$$
\left|A_{\sigma 1}^{(n)}\right|=\Omega\left(\left|T_{n}^{\prime}\right|\right) ; \quad\left|A_{\sigma 2}^{(n)}\right|=\Omega\left(\left|T_{n}^{\prime}\right|\right) ;
$$

Fix $\sigma \in \Sigma^{*}$ with $|\sigma|=k-1$ and note that

$$
\begin{aligned}
& N^{+}\left(A_{\sigma 1}^{(n)}\right) \cup N^{-}\left(A_{\sigma 1}^{(n)}\right)=V\left(T_{n}^{\prime}\right) \backslash A_{\sigma 1}^{(n)} ; \\
& N^{+}\left(A_{\sigma 2}^{(n)}\right) \cup N^{-}\left(A_{\sigma 2}^{(n)}\right)=V\left(T_{n}^{\prime}\right) \backslash A_{\sigma 2}^{(n)} .
\end{aligned}
$$

Furthermore, since $\Delta_{t}\left(A^{(n)}\right)=0$ for every $t \leqslant k-1$, we also have

$$
\begin{aligned}
& \left|N^{+}\left(A_{\sigma 1}^{(n)}\right)\right|-\left|N^{-}\left(A_{\sigma 1}^{(n)}\right)\right|=\left|A_{\sigma 2}^{(n)}\right|-\left|A_{\sigma 3}^{(n)}\right| ; \\
& \left|N^{+}\left(A_{\sigma 2}^{(n)}\right)\right|-\left|N^{-}\left(A_{\sigma 2}^{(n)}\right)\right|=\left|A_{\sigma 3}^{(n)}\right|-\left|A_{\sigma 1}^{(n)}\right| .
\end{aligned}
$$

Applying Lemma 41 to $\left(A_{\sigma 1}^{(n)}\right)_{n \in \mathbb{N}}$ and $\left(A_{\sigma 2}^{(n)}\right)_{n \in \mathbb{N}}$, we get

$$
\begin{gathered}
\left|A_{\sigma 2}^{(n)}\right|-\left|A_{\sigma 3}^{(n)}\right|=o\left(\left|T_{n}^{\prime}\right|\right) ; \\
\left|A_{\sigma 3}^{(n)}\right|-\left|A_{\sigma 1}^{(n)}\right|=o\left(\left|T_{n}^{\prime}\right|\right) .
\end{gathered}
$$

Since $\sigma$ was chosen arbitrarily, we conclude that $\Delta_{k}\left(A^{(n)}\right)=o\left(\left|T_{n}^{\prime}\right|\right)$. This means that we can edit $o\left(\left|T_{n}^{\prime}\right|^{2}\right)$ arcs of $T_{n}^{\prime}$ and obtain a $k$-equally $\vec{C}_{3}$-decomposable tournament $T_{n}^{(k)}$ (note that it is crucial that $\left|T_{n}^{\prime}\right|$ is a power of 3 ) and since this doesn't affect the convergence of the sequence $\left(T_{n}^{\prime}\right)_{n \in \mathbb{N}}$, the sequence $\left(T_{n}^{(k)}\right)_{n \in \mathbb{N}}$ also converges to $\phi$ and we still have that $\left|T_{n}^{(k)}\right|$ is a power of 3 .

Lemma 44. We have $L_{5} \Longrightarrow L_{1}$.

Proof. For every $k \in \mathbb{N}$, let $\left(T_{n}^{(k)}\right)_{n \in \mathbb{N}}$ be a sequence of $k$-equally $\vec{C}_{3}$-decomposable tournaments converging to $\phi$. 
Our objective is to diagonalize the family of sequences $\left(T_{n}^{(k)}\right)_{n \in \mathbb{N}}$ in a way that the resulting sequence still converges to $\phi$. To do this, we let $\left(D_{t}\right)_{t \in \mathbb{N}}$ be an enumeration of the set of all finite tournaments $\mathcal{T}$, we set $f(0)=0$, and for every $k>0$, we let

$$
f(k)=\min \left\{u \in \mathbb{N}:\left|T_{u}^{(k)}\right|>\left|T_{f(k-1)}^{(k-1)}\right| \wedge \forall t \leqslant k, \forall m \geqslant u,\left|p\left(D_{t} ; T_{m}^{(k)}\right)-\phi\left(D_{t}\right)\right|<\frac{1}{k}\right\} .
$$

Note that the fact that $\left(T_{n}^{(k)}\right)_{n \in \mathbb{N}}$ converges to $\phi$ guarantees that $f(k)<\infty$ for every $k \in \mathbb{N}$.

Define now the sequence of tournaments $\left(U_{n}\right)_{n \in \mathbb{N}}$ by letting $U_{n}=T_{f(n)}^{(n)}$ for every $n \in \mathbb{N}$.

We claim that $\left(U_{n}\right)_{n \in \mathbb{N}}$ also converges to $\phi$. Indeed, if $T^{\prime} \in \mathcal{T}$ is a tournament, then there exists $t \in \mathbb{N}$ such that $D_{t}=T^{\prime}$, hence, for every $n>t$, we have

$$
\left|p\left(D_{t} ; U_{n}\right)-\phi\left(D_{t}\right)\right|<\frac{1}{n},
$$

which implies that $\lim _{n \rightarrow \infty} p\left(D_{t} ; U_{n}\right)=\phi\left(D_{t}\right)$. Therefore $\left(U_{n}\right)_{n \in \mathbb{N}}$ converges to $\phi$.

By construction, we know $U_{n}$ is $n$-equally $\vec{C}_{3}$-decomposable; this means that we can obtain $\vec{C}_{\left|U_{n}\right|}^{3}$ from $U_{n}$ by editing at most

$$
\left(\begin{array}{c}
\left|U_{n}\right| \\
2
\end{array}\right)-\sum_{i=1}^{n} 3^{i}\left(\frac{\left|U_{n}\right|}{3^{i}}\right)^{2}=\left(\begin{array}{c}
\left|U_{n}\right| \\
2
\end{array}\right)-\left|U_{n}\right|^{2}\left(\frac{1-3^{-n}}{2}\right)=o\left(\left|U_{n}\right|^{2}\right)
$$

$\operatorname{arcs}$ of $U_{n}$.

Therefore the sequence $\left(\vec{C}_{\left|U_{n}\right|}^{3}\right)_{n \in \mathbb{N}}$ also converges to $\phi$, and since it is a subsequence of $\left(\vec{C}_{n}^{3}\right)_{n \in \mathbb{N}}$, we have $\phi=\phi_{\vec{C}_{3}}$.

Finally, we prove the convergence of the sequence $\left(\vec{C}_{n}^{3}\right)_{n \in \mathbb{N}}$. This proof can be obtained by reinterpreting the proofs of Lemmas 43 and 44 .

Proposition 45. The sequence $\left(\vec{C}_{n}^{3}\right)_{n \in \mathbb{N}}$ is convergent.

Proof. Let

$$
\mathcal{C}=\left\{I \subset \mathbb{N}:\left(\vec{C}_{i}^{3}\right)_{i \in I} \text { is convergent }\right\}
$$

and for every $I \in \mathcal{C}$, let $\phi_{I}$ denote the limit of $\left(\vec{C}_{i}^{3}\right)_{i \in I}$.

From compactness of $[0,1]^{\mathcal{T}}$, we know that $\mathcal{C} \neq \varnothing$. Even more, from compactness of $[0,1]^{\mathcal{T}}$, we know that there exists $I_{0} \in \mathcal{C}$ such that

$$
I_{0} \subset\left\{3^{n}: n \in \mathbb{N}\right\}
$$

Note that if $I \in \mathcal{C}$, then $\phi_{I}$ is $\vec{C}_{3}$-decomposable (since the $\vec{C}_{n}^{3}$ are $\vec{C}_{3}$-decomposable) and balanced (since all vertices of the $\vec{C}_{3}$ have outdegree either $\left\lfloor\left|\vec{C}_{n}^{3}\right| / 2\right\rfloor$ or $\left\lceil\left|\vec{C}_{n}^{3}\right| / 2\right\rceil$ ). Therefore $\phi_{I}$ satisfies $L_{4}$, for every $I \in \mathcal{C}$. 
Now we repeat the proof of Lemma 43 for each $I \in \mathcal{C}$ to obtain sequences $\left(T_{n}^{(k)}\right)_{n \in \mathbb{N}}$ of $k$-equally $\vec{C}_{3}$-decomposable tournaments converging to $\phi_{I}$ for each $k \in \mathbb{N}$. However, we require that these sequences are such that $\left|T_{n}^{(k)}\right| \in I_{0}$ for every $n, k \in \mathbb{N}$.

We proceed then to the proof of Lemma 44 and we get that $\phi_{I}$ is also the limit of a subsequence of $\left(\vec{C}_{i}^{3}\right)_{i \in I_{0}}$, hence $\phi_{I}=\phi_{I_{0}}$ for every $I \in \mathcal{C}$.

Therefore, every convergent subsequence of $\left(\vec{C}_{n}^{3}\right)_{n \in \mathbb{N}}$ converges to the same limit $\phi_{I_{0}}$. By compactness of $[0,1]^{\mathcal{T}}$ again, this implies that $\left(\vec{C}_{n}^{3}\right)_{n \in \mathbb{N}}$ is convergent.

\section{$7 \quad$ Proof of Theorem 33}

For convenience of the reader we state the theorem again below.

Theorem. A tournament $T$ is $\vec{C}_{3}$-decomposable if and only if it has no copies of $T_{5}^{8}, T_{5}^{10}$ nor of $T_{5}^{12}$.

Proof. It is straightforward to check that $T_{5}^{8}, T_{5}^{10}$ and $T_{5}^{12}$ are not $\vec{C}_{3}$-decomposable and that the property of $\vec{C}_{3}$-decomposability is hereditary (i.e., every subtournament of a $\vec{C}_{3^{-}}$ decomposable tournament is also $\vec{C}_{3}$-decomposable). This concludes the proof for one direction.

We will prove the other direction by induction in the size $n$ of the tournament $T$ with no copies of $T_{5}^{8}, T_{5}^{10}$ nor of $T_{5}^{12}$.

If $n \leqslant 2$, then trivially $T$ is $\vec{C}_{3}$-decomposable. So let $n \geqslant 3$ and suppose the assertion is true for tournaments of size smaller than $n$.

If $T$ is transitive, then we can let $A$ be the singleton consisting of the vertex of $T$ with maximum outdegree, let $B=V(T) \backslash A$ and $C=\varnothing$ and note that $(A, B, C)$ satisfies the items in Definition 31 (using inductive hypothesis for item (iv)), hence $T$ is $\vec{C}_{3^{-}}$ decomposable.

Suppose then that $T$ is not transitive and let $a, b, c \in V(T)$ be such that $a b, b c, c a \in$ $A(T)$.

Define the following sets

$$
\begin{array}{rlrl}
V_{a b c} & =\{v \in V(T): v a, v b, v c \in A(T)\} ; & & V_{a b}=\{v \in V(T): v a, v b, c v \in A(T)\} ; \\
V_{b c} & =\{v \in V(T): a v, v b, v c \in A(T)\} ; & V_{a c} & =\{v \in V(T): v a, b v, v c \in A(T)\} ; \\
V_{a} & =\{v \in V(T): v a, b v, c v \in A(T)\} ; & V_{b}=\{v \in V(T): a v, v b, c v \in A(T)\} ; \\
V_{c}=\{v \in V(T): a v, b v, v c \in A(T)\} ; & V_{\varnothing}=\{v \in V(T): a v, b v, c v \in A(T)\} ;
\end{array}
$$

and note that these sets form a partition of $V(T) \backslash\{a, b, c\}$.

We may suppose furthermore that $(a, b, c)$ is chosen in such a way as to minimize $\mid V_{a b c} \cup$ $V_{\varnothing} \mid$.

We claim now the following assertions (see Figure 8).

a. $V_{a b} \times V_{b c}, V_{b c} \times V_{a c}, V_{a c} \times V_{a b} \subset A(T)$, otherwise there would exist a copy of $T_{5}^{10}$ in $T$; 


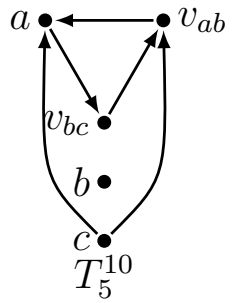

(a) Copy of $T_{5}^{10}$ in $T$ in item (a) if $v_{a b} \in$ $V_{a b}$ and $v_{b c} \in V_{b c}$ are such that $v_{b c}$ beats $v_{a b}$.

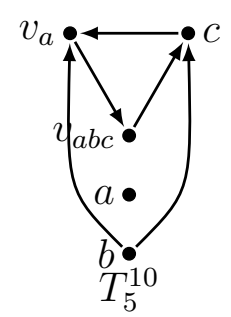

(e) Copy of $T_{5}^{10}$ in $T$ in item (e) if $v_{a b c} \in$ $V_{a b c}$ and $v_{a} \in V_{a}$ are such that $v_{a}$ beats $v_{a b c}$.

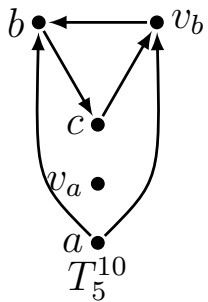

(b) Copy of $T_{5}^{10}$ in $T$ in item (b) if $v_{a} \in$ $V_{a}$ and $v_{b} \in V_{b}$ are such that $v_{b}$ beats $v_{a}$.

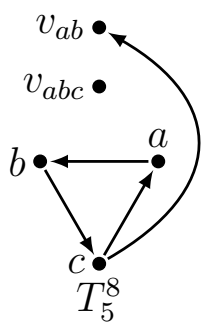

(f) Copy of $T_{5}^{8}$ in $T$ in item (f) if $v_{a b c} \in V_{a b c}$ and $v_{a b} \in V_{a b}$ are such that $v_{a b}$ beats $v_{a b c}$.

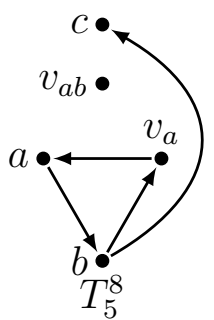

(c) Copy of $T_{5}^{8}$ in $T$ in item (c) if $v_{a} \in$ $V_{a}$ and $v_{a b} \in V_{a b}$ are such that $v_{a b}$ beats $v_{a}$.

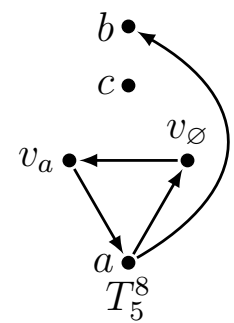

(g) Copy of $T_{5}^{8}$ in $T$ in item (g) if $v_{a} \in$ $V_{a}$ and $v_{\varnothing} \in V_{\varnothing}$ are such that $v_{\varnothing}$ beats $v_{a}$.

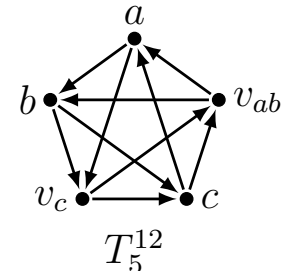

(d) Copy of $T_{5}^{12}$ in $T$ in item (d) if $v_{a b} \in$ $V_{a b}$ and $v_{c} \in V_{c}$ are such that $v_{c}$ beats $v_{a b}$.

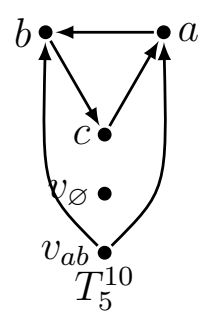

(h) Copy of $T_{5}^{10}$ in $T$ in item (h) if $v_{a b} \in$ $V_{a b}$ and $v_{\varnothing} \in V_{\varnothing}$ are such that $v_{\varnothing}$ beats $v_{a b}$.

Figure 8: Contradictions of the proof of Theorem 33 involving arcs between the sets $V_{a b c}$, $V_{a b}, V_{b c}, V_{a c}, V_{a}, V_{b}, V_{c}$ and $V_{\varnothing}$ and forbidden tournaments $T_{5}^{8}, T_{5}^{10}$ and $T_{5}^{12}$. The arcs omitted are all oriented downward.

b. $V_{a} \times V_{b}, V_{b} \times V_{c}, V_{c} \times V_{a} \subset A(T)$, otherwise there would exist a copy of $T_{5}^{10}$ in $T$;

c. $V_{a} \times V_{a b}, V_{b} \times V_{b c}, V_{c} \times V_{a c} \subset A(T)$, otherwise there would exist a copy of $T_{5}^{8}$ in $T$;

d. $V_{a b} \times V_{c}, V_{b c} \times V_{a}, V_{a c} \times V_{b} \subset A(T)$, otherwise there would exist a copy of $T_{5}^{12}$ in $T$;

e. $V_{a b c} \times\left(V_{a} \cup V_{b} \cup V_{c}\right) \subset A(T)$, otherwise there would exist a copy of $T_{5}^{10}$ in $T$;

f. $V_{a b c} \times\left(V_{a b} \cup V_{b c} \cup V_{a c}\right) \subset A(T)$, otherwise there would exist a copy of $T_{5}^{8}$ in $T$;

g. $\left(V_{a} \cup V_{b} \cup V_{c}\right) \times V_{\varnothing} \subset A(T)$, otherwise there would exist a copy of $T_{5}^{8}$ in $T$;

h. $\left(V_{a b} \cup V_{b c} \cup V_{a c}\right) \times V_{\varnothing} \subset A(T)$, otherwise there would exist a copy of $T_{5}^{10}$ in $T$. 
Now we claim that $V_{a b c} \times V_{\varnothing} \subset A(T)$. Suppose not, that is, suppose that $v_{a b c} \in V_{a b c}$ and $v_{\varnothing} \in V_{\varnothing}$ are such that $v_{\varnothing} v_{a b c} \in A(T)$. Since $v_{a b c} a, a v_{\varnothing} \in A(T)$, we have

$$
\begin{aligned}
\{v \in V(T) & \left.: v a, v v_{a b c}, v v_{\varnothing} \in A(T)\right\} \\
& \cup\left\{v \in V(T): a v, v_{a b c} v, v_{\varnothing} v \in A(T)\right\} \subset\left(V_{a b c} \cup V_{\varnothing}\right) \backslash\left\{v_{a b c}, v_{\varnothing}\right\},
\end{aligned}
$$

contradicting the choice of $(a, b, c)$ such as to minimize $\left|V_{a b c} \cup V_{\varnothing}\right|$. Therefore we must have $V_{a b c} \times V_{\varnothing} \subset A(T)$.

Figure 9 shows all arcs of $T$ proven so far.

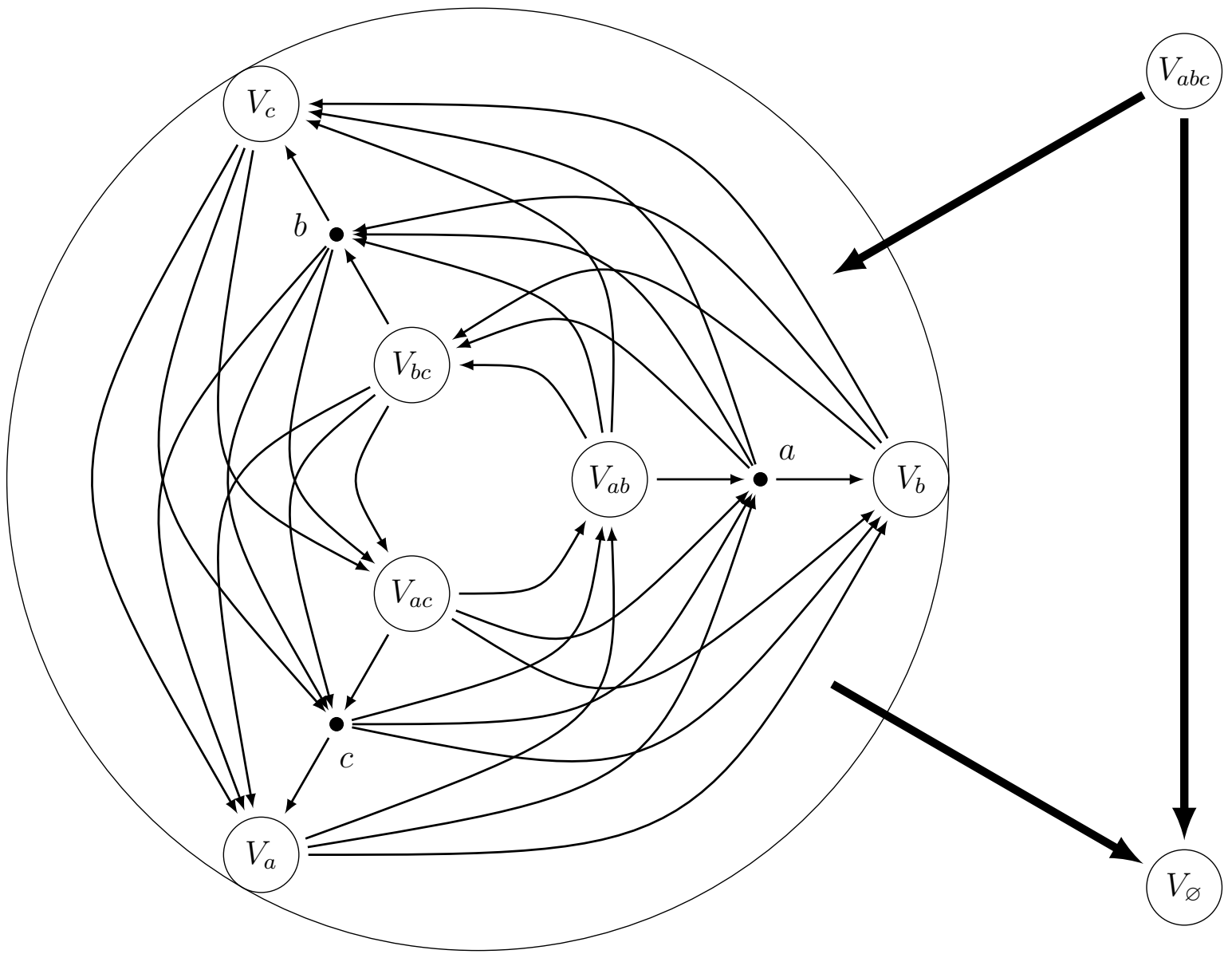

Figure 9: Typical structure of $T$ in the proof of Theorem 33.

Finally, we consider three cases.

If $V_{a b c} \neq \varnothing$, let $A=V_{a b c}, B=V(T) \backslash V_{a b c}$ and $C=\varnothing$ and note that $(A, B, C)$ satisfies the items in Definition 31 (using inductive hypothesis for item (iv)), hence $T$ is $\vec{C}_{3}$-decomposable.

If $V_{\varnothing} \neq \varnothing$, let $A=V(T) \backslash V_{\varnothing}, B=V_{\varnothing}$ and $C=\varnothing$ and note that $(A, B, C)$ satisfies the items in Definition 31 (using inductive hypothesis for item (iv)), hence $T$ is $\vec{C}_{3^{-}}$ decomposable. 
And finally, if $V_{a b c} \cup V_{\varnothing}=\varnothing$, let

$$
A=\{a\} \cup V_{b} \cup V_{a b} ; \quad B=\{b\} \cup V_{c} \cup V_{b c} ; \quad C=\{c\} \cup V_{a} \cup V_{a c} ;
$$

and note that $(A, B, C)$ satisfies the items in Definition 31 (using inductive hypothesis for item (iv)), hence $T$ is $\vec{C}_{3}$-decomposable.

\section{$8 \quad$ Proof of Lemma 42}

For convenience of the reader we state the lemma again below.

Lemma. If $\left(T_{n}\right)_{n \in \mathbb{N}}$ is a sequence of $\vec{C}_{3}$-decomposable tournaments that converges to a balanced homomorphism $\phi \in \operatorname{Hom}^{+}\left(\mathcal{A}^{0}, \mathbb{R}\right)$, then there exists a sequence $\left(T_{n}^{\prime}\right)_{n \in \mathbb{N}}$ of $\vec{C}_{3^{-}}$ decomposable tournaments and for every $n \in \mathbb{N}$ a $\vec{C}_{3^{-}}$decomposition $\left(A_{\sigma}^{(n)}\right)_{\sigma \in \Sigma^{*}}$ of $T_{n}^{\prime}$ such that

- There exists a subsequence $\left(T_{k_{n}}\right)_{n \in \mathbb{N}}$ of $\left(T_{n}\right)_{n \in \mathbb{N}}$ such that the tournament $T_{n}^{\prime}$ can be obtained from $T_{k_{n}}$ by flipping $o\left(\left|T_{k_{n}}\right|^{2}\right)$ arcs (hence $\left(T_{n}^{\prime}\right)_{n \in \mathbb{N}}$ also converges to $\phi$ );

- We have $\left|A_{1}^{(n)}\right|=\Omega\left(\left|T_{n}^{\prime}\right|\right)$ and $\left|A_{2}^{(n)}\right|=\Omega\left(\left|T_{n}^{\prime}\right|\right)$.

Proof. Suppose the lemma is not true and let $\left(T_{n}\right)_{n \in \mathbb{N}}$ be a counter-example sequence.

For every $n \in \mathbb{N}$, let $\left(B_{\sigma}^{(n)}\right)_{\sigma \in \Sigma^{*}}$ be a $\vec{C}_{3^{-}}$-decomposition of $T_{n}$. Without loss of generality, we may suppose that

$$
\forall n \in \mathbb{N}, \forall \sigma \in \Sigma^{*},\left|B_{\sigma 1}^{(n)}\right| \geqslant\left|B_{\sigma 2}^{(n)}\right| \wedge\left|B_{\sigma 1}^{(n)}\right| \geqslant\left|B_{\sigma 3}^{(n)}\right| .
$$

Before we start the technical part, let us provide some intuition on the structure of the proof. First, we will prove that since $\phi$ is balanced, whenever some $B_{1^{v+1}}^{(n)}$ has non-negligible size, by analyzing its outneighborhood and inneighborhood, we get that $\left|B_{1^{t} 2}^{(n)}\right| \approx\left|B_{1^{t} 3}^{(n)}\right|$ for every $t \leqslant v$ (Claim 46).

Since $T_{n}$ is a counter-example sequence, we know that $B_{2}^{(n)}$ and $B_{3}^{(n)}$ are small. In fact, since we are allowed to flip a negligible amount of arcs, for any constant $c \in \mathbb{N}$, we also know that $B_{1 c 2}^{(n)}$ and $B_{1 c 3}^{(n)}$ must be small, otherwise we could find the first $c$ when one of these are large and flip few arcs involving $\bigcup_{t=0}^{c-1} B_{1^{t} 2}^{(n)}$ and $\bigcup_{t=0}^{c-1} B_{1^{t} 3}^{(n)}$ to merge them into $B_{1^{c} 2}^{(n)}$ and $B_{1^{c} 3}^{(n)}$. Even more, the same argument works to show that if one of $B_{1^{u(n)+12}}^{(n)}$ or $B_{1^{u(n)+13}}^{(n)}$ is large then $\bigcup_{t=0}^{u(n)} B_{1^{t} 2}^{(n)} \cup B_{1^{t} 3}^{(n)}$ must be large as well (Claim 47). So we can collect the vertices in these sets until we have a large amount of vertices by letting $v(n)$ be the first point when $\bigcup_{t=0}^{v(n)} B_{1^{t} 2}^{(n)} \cup B_{1^{t} 3}^{(n)}$ is large. Since this is the first point when this is large, the set $B_{1^{v(n)+1}}^{(n)}$ is also large, hence $\bigcup_{t=0}^{v(n)} B_{1^{t} 2}^{(n)}$ and $\bigcup_{t=0}^{v(n)} B_{1^{t} 3}^{(n)}$ have approximately the same size, i.e., they are both large.

Since $\bigcup_{t=0}^{v(n)} B_{1^{t} 2}^{(n)}$ is large, there must exist some $w(n) \leqslant v(n)$ such that $B_{1^{w(n)} 2}^{(n)}$ is large (Claim 48). By repeating the first part of the argument, the union $\bigcup_{t=0}^{w(n)-1} B_{1^{t 2}}^{(n)}$ must be large, which implies that $B_{1^{u(n)} 2}^{(n)}$ is large for some $u(n) \leqslant w(n)-1$. 
A priori, the set $B_{1^{u(n)} 2}^{(n)}$ does not need to be asymptotically as large as $B_{1^{w(n)} 2}^{(n)}$, but since $\phi$ is balanced, we have $\left|B_{1^{u(n)+1}}^{(n)}\right| \approx\left|B_{1^{u(n)} 2}^{(n)}\right|$, which implies that $B_{1^{u(n)} 2}^{(n)}$ has asymptotic size at least $3\left|B_{1^{w(n)} 2}^{(n)}\right|($ Claim 49). This means that if we started our choice of $w(n)$ as the first point when $B_{1^{w(n)} 2}^{(n)}$ is, say, at least $\epsilon\left|T_{n}\right|$, then $\left|B_{1^{u(n) 2}}^{(n)}\right| \geqslant 2.9 \epsilon\left|T_{n}\right|$ when $n$ is large enough, which is a contradiction.

Let us now formalize the argument above, starting with all the claims.

Claim 46. Suppose $u, v: \mathbb{N} \rightarrow \mathbb{N}$ are two functions such that $u(n) \leqslant v(n)$ for every $n \in \mathbb{N}$. If $\left|B_{1^{v(n)+1}}^{(n)}\right|=\Omega\left(\left|T_{n}\right|\right)$, then

$$
\left|\bigcup_{t=u(n)}^{v(n)} B_{1^{t} 2}^{(n)}\right|-\left|\bigcup_{t=u(n)}^{v(n)} B_{1^{t} 3}^{(n)}\right|=o\left(\left|T_{n}\right|\right)
$$

Proof. Note that

$$
N^{+}\left(B_{1^{v(n)+1}}^{(n)}\right)=\bigcup_{t=0}^{v(n)} B_{1^{t} 2}^{(n)} ; \quad N^{-}\left(B_{1^{v(n)+1}}^{(n)}\right)=\bigcup_{t=0}^{v(n)} B_{1^{t} 3}^{(n)} .
$$

Since $\left|B_{1^{v(n)+1}}^{(n)}\right|=\Omega\left(\left|T_{n}\right|\right)$, by Lemma 41 , we have

$$
\left|\bigcup_{t=0}^{v(n)} B_{1^{t} 2}^{(n)}\right|-\left|\bigcup_{t=0}^{v(n)} B_{1^{t} 3}^{(n)}\right|=o\left(\left|T_{n}\right|\right) .
$$

Note that, since $u(n) \leqslant v(n)$, we have $B_{1^{v(n)+1}}^{(n)} \subset B_{1^{u(n)}}^{(n)}$, which implies $\left|B_{1^{u(n)}}^{(n)}\right|=$ $\Omega\left(\left|T_{n}\right|\right)$, hence we have

$$
\left|\bigcup_{t=0}^{u(n)-1} B_{1^{t} 2}^{(n)}\right|-\left|\bigcup_{t=0}^{u(n)-1} B_{1^{t} 3}^{(n)}\right|=o\left(\left|T_{n}\right|\right)
$$

analogously to the case with $v(n)$.

The result follows by subtracting equation (8) from equation (7).

Claim 47. If $u: \mathbb{N} \rightarrow \mathbb{N}$ is a function such that $\left|B_{1^{u(n)+12} 2}^{(n)}\right|=\Omega\left(\left|T_{n}\right|\right)$, then

$$
\left|\bigcup_{t=0}^{u(n)} B_{1^{t 2}}^{(n)}\right|=\Omega\left(\left|T_{n}\right|\right)
$$

Proof. Suppose the claim is not true. This means that there is a subsequence $\left(T_{k_{n}}\right)_{n \in \mathbb{N}}$ of $\left(T_{n}\right)_{n \in \mathbb{N}}$ such that

$$
\left|\bigcup_{t=0}^{u\left(k_{n}\right)} B_{1^{t} 2}^{\left(k_{n}\right)}\right|=o\left(\left|T_{k_{n}}\right|\right)
$$


Let $T_{n}^{\prime}$ be the tournament obtained from $T_{k_{n}}$ by flipping all the arcs in

$$
\begin{aligned}
A\left(T_{k_{n}}\right) \cap & \left(\left(B_{1^{u\left(k_{n}\right)+13}}^{\left(k_{n}\right)} \times\left(\bigcup_{t=0}^{u\left(k_{n}\right)} B_{1^{t} 2}^{\left(k_{n}\right)}\right)\right)\right. \\
& \cup\left(\left(\bigcup_{t=0}^{u\left(k_{n}\right)} B_{1^{t} 3}^{\left(k_{n}\right)}\right) \times\left(\bigcup_{t=0}^{u\left(k_{n}\right)} B_{1^{t} 2}^{\left(k_{n}\right)}\right)\right) \\
& \left.\cup\left(\left(\bigcup_{t=0}^{u\left(k_{n}\right)} B_{1^{t} 3}^{\left(k_{n}\right)}\right) \times B_{1^{u\left(k_{n}\right)+12}}^{\left(k_{n}\right)}\right)\right)
\end{aligned}
$$

and note that equation (9) and Claim 46 imply that the total of arcs flipped is $o\left(\left|T_{k_{n}}\right|^{2}\right)$.

Let

$$
A_{1}^{(n)}=B_{1^{u\left(k_{n}\right)+2}}^{\left(k_{n}\right)} ; \quad A_{2}^{(n)}=\bigcup_{t=0}^{u\left(k_{n}\right)+1} B_{1^{t} 2}^{\left(k_{n}\right)} ; \quad A_{3}^{(n)}=\bigcup_{t=0}^{u\left(k_{n}\right)+1} B_{1^{t} 3}^{\left(k_{n}\right)}
$$

and note that $\left|A_{1}^{(n)}\right|+o\left(\left|T_{n}\right|\right) \geqslant\left|A_{2}^{(n)}\right| \geqslant \Omega\left(\left|T_{n}^{\prime}\right|\right)$.

Completing $\left(A_{1}^{(n)}, A_{2}^{(n)}, A_{3}^{(n)}\right)$ to a $\vec{C}_{3}$-decomposition of $T_{n}^{\prime}$ contradicts the choice of $\left(T_{n}\right)_{n \in \mathbb{N}}$ as a counter-example sequence.

Claim 48. If $u: \mathbb{N} \rightarrow \mathbb{N}$ is a function such that $\left|B_{1^{u(n)+1}}^{(n)}\right|=\Omega\left(\left|T_{n}\right|\right)$ and

$$
\left|\bigcup_{t=0}^{u(n)} B_{1^{t 2}}^{(n)}\right|=\Omega\left(\left|T_{n}\right|\right)
$$

then there exists a function $w: \mathbb{N} \rightarrow \mathbb{N}$ such that $w(n) \leqslant u(n)$ and $\left|B_{1^{w(n)} 2}^{(n)}\right|=\Omega\left(\left|T_{n}\right|\right)$.

Proof. For every $n \in \mathbb{N}$, let

$$
\begin{aligned}
& M(n)=\max \left\{\left|B_{1^{w} 2}^{(n)}\right|: w \leqslant u(n)\right\} \\
& R(n)=\max \left\{\left|\bigcup_{t=0}^{r} B_{1^{t} 2}^{(n)}\right|-\left|\bigcup_{t=0}^{r} B_{1^{t} 3}^{(n)}\right|: r \leqslant u(n)\right\} ; \\
& S(n)=\max \left\{\left|B_{1^{s} 3}^{(n)}\right|-\left|B_{1^{s} 2}^{(n)}\right|: s \leqslant u(n)\right\} .
\end{aligned}
$$

By Claim 46, we know that $R(n)=o\left(\left|T_{n}\right|\right)$ and $S(n)=o\left(\left|T_{n}\right|\right)$.

Suppose towards a contradiction that the claim is false. This means that we must have $M(n) \neq \Omega\left(\left|T_{n}\right|\right)$, that is, there exists a subsequence $\left(T_{k_{n}}\right)_{n \in \mathbb{N}}$ of $\left(T_{n}\right)_{n \in \mathbb{N}}$ such that $M\left(k_{n}\right)=o\left(\left|T_{k_{n}}\right|\right)$. 
Note now that if $t \leqslant u\left(k_{n}\right)$ and $v_{t} \in B_{1 t 2}^{\left(k_{n}\right)}$ (see Figure 10 further ahead for the neighbourhoods of the set $\left.B_{1^{t} 2}^{\left(k_{n}\right)}\right)$, then

$$
\begin{aligned}
& d^{+}\left(v_{t}\right)=\left|\bigcup_{i=0}^{t-1} B_{1^{i} 2}^{\left(k_{n}\right)}\right|+\left|B_{1^{t} 3}^{\left(k_{n}\right)}\right|+d_{B_{1^{2} 2}^{\left(k_{n}\right)}}^{+}\left(v_{t}\right) ; \\
& d^{-}\left(v_{t}\right)=\left|\bigcup_{i=0}^{t-1} B_{1^{i} 3}^{\left(k_{n}\right)}\right|+\left|B_{1^{t+1}}^{\left(k_{n}\right)}\right|+d_{B_{1^{t} 2}^{\left(k_{n}\right)}}^{-}\left(v_{t}\right),
\end{aligned}
$$

where $d_{A}^{+}(v)=\left|N^{+}(v) \cap A\right|$ and $d_{A}^{-}(v)=\left|N^{-}(v) \cap A\right|$.

Since

$$
\begin{aligned}
\left|\bigcup_{i=0}^{t-1} B_{1^{i} 2}^{\left(k_{n}\right)}\right|-\left|\bigcup_{i=0}^{t-1} B_{1^{i} 3}^{\left(k_{n}\right)}\right| & \leqslant R\left(k_{n}\right) \\
\left|B_{1^{t} 3}^{\left(k_{n}\right)}\right| & \leqslant\left|B_{1^{t 2}}^{\left(k_{n}\right)}\right|+S\left(k_{n}\right) \leqslant M\left(k_{n}\right)+S\left(k_{n}\right) \\
d_{B_{1^{t} 2}^{\left(k_{n}\right)}}^{+}\left(v_{t}\right)-d_{B_{1^{t}}^{\left(k_{n}\right)}}^{-}\left(v_{t}\right) & \leqslant\left|B_{1^{t} 2}^{\left(k_{n}\right)}\right| \leqslant M\left(k_{n}\right) \\
\left|B_{1^{t+1}}^{\left(k_{n}\right)}\right| & \geqslant\left|B_{1^{u\left(k_{n}\right)+1}}^{\left(k_{n}\right)}\right|
\end{aligned}
$$

we have

$$
d^{+}\left(v_{t}\right)-d^{-}\left(v_{t}\right) \leqslant R\left(k_{n}\right)+2 M\left(k_{n}\right)+S\left(k_{n}\right)-\left|B_{1^{u\left(k_{n}\right)+1}}^{\left(k_{n}\right)}\right| .
$$

Note that this bound does not depend on $t$.

Since $R\left(k_{n}\right)=o\left(\left|T_{k_{n}}\right|\right), M\left(k_{n}\right)=o\left(\left|T_{k_{n}}\right|\right), S\left(k_{n}\right)=o\left(\left|T_{k_{n}}\right|\right)$ and $\left|B_{1^{u\left(k_{n}\right)+1}}^{\left(k_{n}\right)}\right|=\Omega\left(\left|T_{k_{n}}\right|\right)$, this implies that

$$
d^{+}(v)-d^{-}(v) \leqslant R\left(k_{n}\right)+2 M\left(k_{n}\right)+S\left(k_{n}\right)-\left|B_{1^{u\left(k_{n}\right)+1}}^{\left(k_{n}\right)}\right| \leqslant-\epsilon\left|T_{k_{n}}\right|,
$$

for every $v \in \bigcup_{t=0}^{u\left(k_{n}\right)} B_{1^{t} 2}^{\left(k_{n}\right)}$ and $n \in \mathbb{N}$ large enough, which contradicts the fact that $\phi$ is balanced (since $\left|\bigcup_{t=0}^{u\left(k_{n}\right)} B_{1^{t} 2}^{\left(k_{n}\right)}\right|=\Omega\left(\left|T_{k_{n}}\right|\right)$ ).

Claim 49. Suppose $u, v: \mathbb{N} \rightarrow \mathbb{N}$ are two functions such that $u(n)<v(n)$ for every $n \in \mathbb{N}$. If $\left|B_{1^{u(n) 2}}^{(n)}\right|=\Omega\left(\left|T_{n}\right|\right)$ and $\left|B_{1^{v(n)} 2}^{(n)}\right|=\Omega\left(\left|T_{n}\right|\right)$, then

$$
\left|B_{1^{u(n) 2}}^{(n)}\right|=2\left|\bigcup_{t=u(n)+1}^{v(n)-1} B_{1^{t} 2}^{(n)}\right|+3\left|B_{1^{v(n)} 2}^{(n)}\right|+o\left(\left|T_{n}\right|\right) .
$$

Proof. By Lemma 41, we know that $\left|N^{+}\left(B_{1^{v(n)} 2}^{(n)}\right)\right|-\left|N^{-}\left(B_{1^{v(n)} 2}^{(n)}\right)\right|=o\left(\left|T_{n}\right|\right)$, that is, we have (see Figure 10)

$$
\left|\bigcup_{t=0}^{v(n)-1} B_{1^{t} 2}^{(n)}\right|+\left|B_{1^{v(n)} 3}^{(n)}\right|-\left|\bigcup_{t=0}^{v(n)-1} B_{1^{t} 3}^{(n)}\right|-\left|B_{1^{v(n)+1}}^{(n)}\right|=o\left(\left|T_{n}\right|\right) .
$$




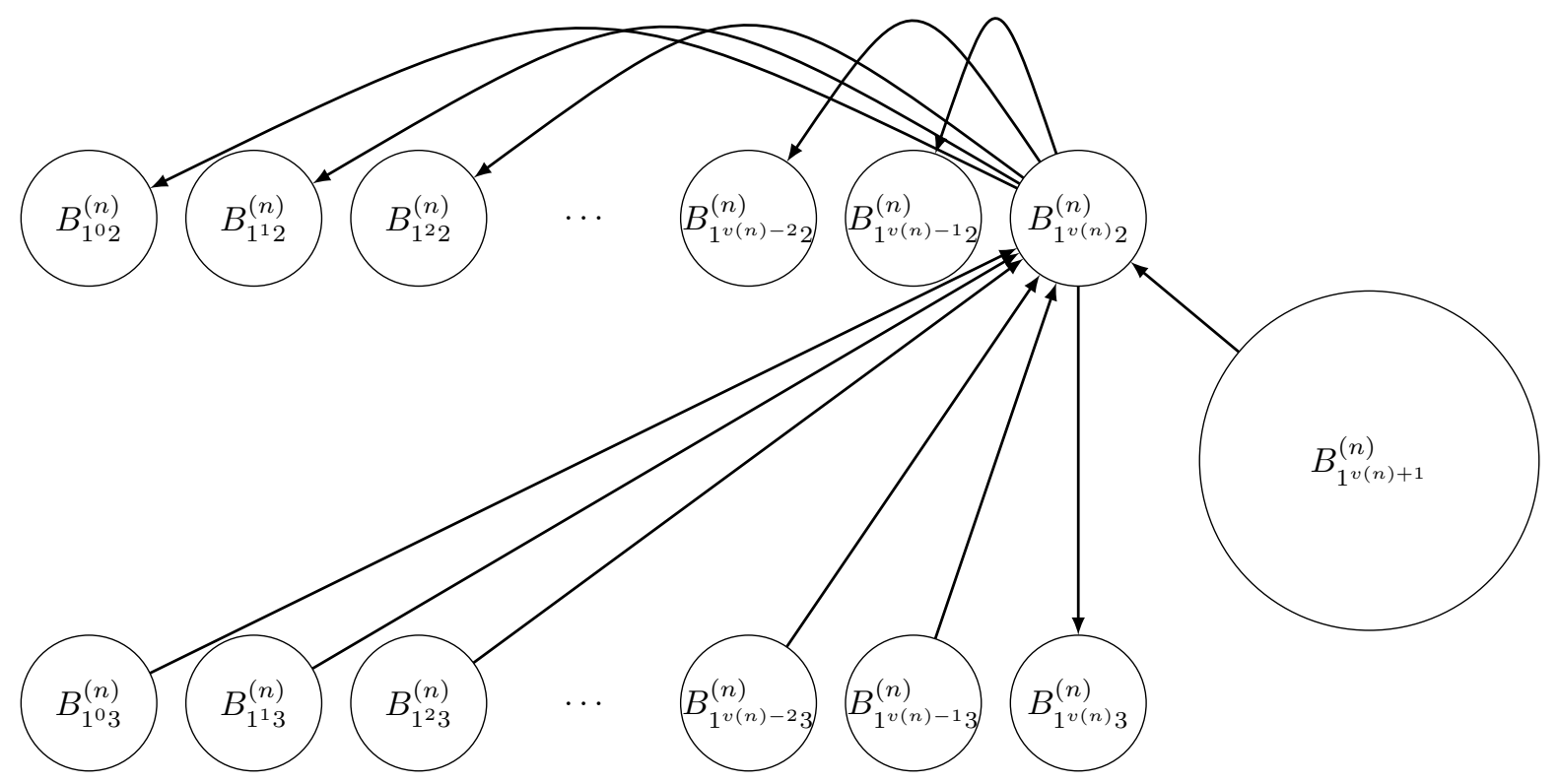

Figure 10: Neighbourhoods of $B_{1^{v(n)} 2}^{(n)}$.

By Claim 46, this implies that

$$
\left|B_{1^{v(n)} 2}^{(n)}\right|-\left|B_{1^{v(n)+1}}^{(n)}\right|=o\left(\left|T_{n}\right|\right) .
$$

With an analogous argument for $u(n)$, we get

$$
\left|B_{1^{u(n)} 2}^{(n)}\right|-\left|B_{1^{u(n)+1}}^{(n)}\right|=o\left(\left|T_{n}\right|\right),
$$

which implies

$$
\left|B_{1^{u(n) 2} 2}^{(n)}\right|-\left|\bigcup_{t=u(n)+1}^{v(n)-1}\left(B_{1^{t} 2}^{(n)} \cup B_{1^{t} 3}^{(n)}\right)\right|-\left|B_{1^{v(n)+1}}^{(n)}\right|-\left|B_{1^{v(n) 2}}^{(n)}\right|-\left|B_{1^{v(n)} 3}^{(n)}\right|=o\left(\left|T_{n}\right|\right) .
$$
yield

Since $\left|B_{1^{v(n)}}^{(n)}\right| \geqslant\left|B_{1^{v(n)+1}}^{(n)}\right| \geqslant\left|B_{1^{v(n)} 2}^{(n)}\right|=\Omega\left(\left|T_{n}\right|\right)$, two more applications of Claim 46

$$
\left|B_{1^{u(n) 2}}^{(n)}\right|-2\left|\bigcup_{t=u(n)+1}^{v(n)-1} B_{1^{t 2}}^{(n)}\right|-\left|B_{1^{v(n)+1}}^{(n)}\right|-2\left|B_{1^{v(n) 2}}^{(n)}\right|=o\left(\left|T_{n}\right|\right)
$$

Subtracting equation (10) from (11), we get

$$
\left|B_{1^{u(n) 2}}^{(n)}\right|-2\left|\bigcup_{t=u(n)+1}^{v(n)} B_{1^{t 2}}^{(n)}\right|-3\left|B_{1^{v(n)} 2}^{(n)}\right|=o\left(\left|T_{n}\right|\right) .
$$


We are now in condition of finishing the proof of the lemma. For every $n \in \mathbb{N}$, let

$$
v(n)=\min \left\{v \in \mathbb{N}:\left|\bigcup_{t=0}^{v}\left(B_{1^{t} 2}^{(n)} \cup B_{1^{t} 3}^{(n)}\right)\right| \geqslant \frac{2}{3}\left|T_{n}\right|\right\} .
$$

Note that $v(n)$ is well-defined for $n \geqslant 3$ and, by Claim 46, we have $\left|\bigcup_{t=0}^{v(n)} B_{1^{t} 2}^{(n)}\right|=\Omega\left(\left|T_{n}\right|\right)$.

Furthermore, note that

$$
\left|B_{1^{v(n)+1}}^{(n)}\right| \geqslant \frac{1}{9}\left|T_{n}\right|
$$

which, by Claim 48, implies that there exists a function $w: \mathbb{N} \rightarrow \mathbb{N}$ with $w(n) \leqslant v(n)$ for every $n \in \mathbb{N}$ and such that $\left|B_{1 w(n)}^{(n)}\right|=\Omega\left(\left|T_{n}\right|\right)$, that is, there exists $n_{0} \in \mathbb{N}$ and $\epsilon>0$ such that

$$
\left|B_{1 w(n) 2}^{(n)}\right| \geqslant \epsilon\left|T_{n}\right|
$$

for every $n \geqslant n_{0}$.

For every $n \in \mathbb{N}$, let

$$
w_{0}(n)=\min \left\{w \in \mathbb{N}:\left|B_{1^{w} 2}^{(n)}\right| \geqslant \epsilon\left|T_{n}\right|\right\}
$$

and note that, for every $n \geqslant n_{0}$, we have that $w_{0}(n)$ is well-defined and $w_{0}(n) \leqslant w(n)$.

Since $\left|B_{\left.1^{w_{0}(n)}\right)_{2}}^{(n)}\right|=\Omega\left(\left|T_{n}\right|\right)$, by Claim 47, we know that

$$
\left|\bigcup_{t=0}^{w_{0}(n)-1} B_{1^{t 2}}^{(n)}\right|=\Omega\left(\left|T_{n}\right|\right)
$$

Another application of Claim 48 yields then a function $u: \mathbb{N} \rightarrow \mathbb{N}$ with $u(n) \leqslant w_{0}(n)-1$ for every $n \in \mathbb{N}$ and such that $\left|B_{1^{u(n)} 2}^{(n)}\right|=\Omega\left(\left|T_{n}\right|\right)$.

Now, by Claim 49, we have

$$
\left|B_{1^{u(n) 2}}^{(n)}\right|=2\left|\bigcup_{t=u(n)+1}^{w_{0}(n)-1} B_{1^{t 2}}^{(n)}\right|+3\left|B_{1^{w_{0}(n) 2}}^{(n)}\right|+o\left(\left|T_{n}\right|\right) \geqslant 3 \epsilon\left|T_{n}\right|+o\left(\left|T_{n}\right|\right),
$$

which implies that for $n \in \mathbb{N}$ large enough, we have $\left|B_{1^{u(n)} 2}^{(n)}\right| \geqslant \epsilon\left|T_{n}\right|$, contradicting the definition of $w_{0}(n)$.

\section{References}

[1] R. Baber, Some results in extremal combinatorics. Thesis (Ph.D.)-University of London, University College London (United Kingdom), 2011. 
[2] J. Balogh, P. Hu, B. Lidický, O. Pikhurko, B. Udvari, and J. Volec, Minimum number of monotone subsequences of length 4 in permutations, Combin. Probab. Comput,. 24:(4):658-679, 2015.

[3] L. W. Beineke and F. Harary, The maximum number of strongly connected subtournaments. Canad. Math. Bull. 8:491-498, 1965.

[4] V. Bhat and V. Rödl, Note on upper density of quasi-random hypergraphs. Electron. J. Combin., 20(2):\#P59, 2013.

[5] B. Borchers, Csdp, a c library for semidefinite programming. Optimization Methods and Software 11(1-4):613-623, 1999.

[6] F. Chung, Quasi-random hypergraphs revisited. Random Structures Algorithms, 40(1):39-48, 2012.

[7] F. Chung and R. Graham, Quasi-random hypergraphs. Random Structures Algorithms 1(1):105-124, 1990.

[8] F. Chung and R. Graham, Quasi-random tournaments. J. Graph Theory 15(2): 173-198, 1991.

[9] F. Chung, R. Graham, and R. Wilson, Quasirandom graphs. Proc. Nat. Acad. Sci. U.S.A., 85(4):969-970, 1988.

[10] U. Colombo, Sui circuiti nei grafi completi. Boll. Un. Mat. Ital., 19(3):153-170, 1964.

[11] J. N. Cooper, Quasirandom permutations. J. Combin. Theory Ser. A, 106(1):123143, 2004.

[12] L. N. Coregliano, Quasi-carousel tournaments. J. Graph Theory, 88(1):192-210, 2018.

[13] L. N. Coregliano and A. Razborov, On the density of transitive tournaments. J. Graph Theory, 85(1):12-21, 2017.

[14] J. Cummings, D. Král', F. Pfender, K. Sperfeld, A. Treglown, and M. Young, Monochromatic triangles in three-coloured graphs. J. Combin. Theory Ser. B, 103(4):489-503, 2013.

[15] S. Das, H. Huang, J. Ma, H. Naves, and B. Sudakov, A problem of Erdös on the minimum number of $k$-cliques. J. Combin. Theory Ser. B, 103(3):344-373, 2013.

[16] P. Diaconis and S. Janson, Graph limits and exchangeable random graphs. Rend. Mat. Appl. 28(1):33-61, 2008.

[17] G. Elek and B. Szegedy, A measure-theoretic approach to the theory of dense hypergraphs. Adv. Math., 231(3-4):1731-1772, 2012.

[18] V. Falgas-Ravry and E. R. Vaughan, Applications of the semi-definite method to the Turán density problem for 3-graphs. Combin. Probab. Comput. 22(1):21-54, 2013.

[19] S. Griffiths, Quasi-random oriented graphs. J. Graph Theory, 74(2):198-203, 2013.

[20] H. Hatami and S. Norine, Undecidability of linear inequalities in graph homomorphism densities. J. Amer. Math. Soc., 24(2):547-565, 2011. 
[21] C. Hoppen, Y. Kohayakawa, C. G. Moreira, B. Ráth, and R. M. Sampaio, Limits of permutation sequences. J. Combin. Theory Ser. B, 103(1):93-113, 2013.

[22] S. Kalyanasundaram and A. Shapira, A note on even cycles and quasirandom tournaments. J. Graph Theory 73(3):260-266, 2013.

[23] D. Král' and O. Pikhurko, Quasirandom permutations are characterized by 4-point densities. Geom. Funct. Anal., 23(2):570-579, 2013.

[24] M. Krivelevich and B. Sudakov, Pseudo-random graphs, More Sets, Graphs and Numbers. Bolyai Society Mathematical Studies, 15:199-262, 2006.

[25] L. Lovász and B. Szegedy, Limits of dense graph sequences. J. Combin. Theory Ser. B, 96(6):933-957, 2006.

[26] Maxima, Maxima, a computer algebra system. version 5.34.1, 2014.

[27] O. Pikhurko and E. R. Vaughan, Minimum number of $k$-cliques in graphs with bounded independence number. Combin. Probab. Comput., 22(6):910-934, 2013.

[28] A. Razborov, Flag algebras J. Symbolic Logic, 72(4):1239-1282, 2007.

[29] A. Razborov, On 3-hypergraphs with forbidden 4-vertex configurations. SIAM J. Discrete Math., 24(3):946-963, 2010.

[30] A. Razborov, Flag algebras: an interim report. The mathematics of Paul Erdös. II, 207-232, 2013.

[31] A. Razborov, What is ... a flag algebra? Notices Amer. Math. Soc., 60(10):13241327, 2013.

[32] A. Thomason, Pseudo-random graphs. Ann. of Discrete Math., 33:307-331, 1987.

[33] M. Yamashita, K. Fujisawa, M. Fukuda, K. Kobayashi, K. Nakata, and M. Nakata, Latest developments in the SDPA family for solving large-scale SDPs. Handbook on semidefinite, conic and polynomial optimization, Internat. Ser. Oper. Res. Management Sci., 166:687-713, 2012.

\section{A Appendix}

\section{A.1 Positive semi-definite matrices used}

$$
Q\left(T_{5}^{7}, 1\right)=\frac{35}{48} \cdot\left(\begin{array}{cccc}
\operatorname{Tr}_{3}^{1, L} & \vec{C}_{3}^{1} & \operatorname{Tr}_{3}^{1, M} & \operatorname{Tr}_{3}^{1, W} \\
1 & -1 & -1 & 1 \\
-1 & 1 & 1 & -1 \\
-1 & 1 & 1 & -1 \\
1 & -1 & -1 & 1
\end{array}\right)=\frac{35}{48} \cdot\left(\begin{array}{c}
1 \\
-1 \\
-1 \\
1
\end{array}\right) \cdot\left(\begin{array}{c}
1 \\
-1 \\
-1 \\
1
\end{array}\right)^{\top}
$$




$$
\begin{aligned}
& Q\left(T_{5}^{7}, \operatorname{Tr}_{3}^{*}\right)=5 \cdot\left(\begin{array}{cccccccc}
\operatorname{Tr}_{4}^{\operatorname{Tr}_{3}^{*}, 3} & W_{4}^{\operatorname{Tr}_{3}^{*}} & \operatorname{Tr}_{4}^{\operatorname{Tr}_{3}^{*}, 2} & R_{4}^{\operatorname{Tr}_{3}^{*}, 1} & L_{4}^{\operatorname{Tr}_{3}^{*}} & \operatorname{Tr}_{4}^{\operatorname{Tr}_{3}^{*}, 1} & R_{4}^{\operatorname{Tr}_{3}^{*}, 2} & \operatorname{Tr}_{4}^{\operatorname{Tr}_{3}^{*}, 0} \\
0 & 0 & -1 & 1 & 0 & 1 & -1 & -1 \\
-1 & 0 & 0 & 0 & 0 & 0 & 0 & 0 \\
1 & 0 & -1 & 1 & 0 & 1 & -1 & -1 \\
0 & 0 & 0 & 0 & 0 & 0 & 0 & 0 \\
1 & 0 & -1 & 1 & 0 & 1 & -1 & -1 \\
-1 & 0 & 1 & -1 & 0 & -1 & 1 & 1 \\
-1 & 0 & 1 & -1 & 0 & -1 & 1 & 1
\end{array}\right) \\
& =5 \cdot\left(\begin{array}{c}
1 \\
0 \\
-1 \\
1 \\
0 \\
1 \\
-1 \\
-1
\end{array}\right) \cdot\left(\begin{array}{c}
1 \\
0 \\
-1 \\
1 \\
0 \\
1 \\
-1 \\
-1
\end{array}\right)^{\top} \\
& Q\left(T_{5}^{7}, \vec{C}_{3}^{*}\right)=12 \cdot\left(\begin{array}{cccccccc}
R_{4}^{\vec{C}_{3}^{*}, 3} & L_{4}^{\vec{C}_{3}^{*}} & R_{4}^{\vec{C}_{3}^{*}, 2} & R_{4}^{\vec{C}_{3}^{*}, 1} & R_{4}^{\vec{C}_{3}^{*}, 23} & W_{4}^{\vec{C}_{3}^{*}} & R_{4}^{\vec{C}_{3}^{*}, 12} & R_{4}^{\vec{C}_{3}^{*}, 13} \\
0 & 0 & 0 & 0 & 0 & 0 & 0 & 0 \\
0 & 1 & 0 & 0 & 0 & -1 & 0 & 0 \\
0 & 0 & 0 & 0 & 0 & 0 & 0 & 0 \\
0 & 0 & 0 & 0 & 0 & 0 & 0 & 0 \\
0 & -1 & 0 & 0 & 0 & 0 & 0 & 0 \\
0 & 0 & 0 & 0 & 0 & 0 & 0 & 0 \\
0 & 0 & 0 & 0 & 0 & 0 & 0 & 0
\end{array}\right) \\
& =12 \cdot\left(\begin{array}{c}
0 \\
1 \\
0 \\
0 \\
0 \\
-1 \\
0 \\
0
\end{array}\right) \cdot\left(\begin{array}{c}
0 \\
1 \\
0 \\
0 \\
0 \\
-1 \\
0 \\
0
\end{array}\right)^{\top} \text {. } \\
& Q\left(T_{5}^{8}, 1\right)=\left(\begin{array}{cccc}
\operatorname{Tr}_{3}^{1, L} & \vec{C}_{3}^{1} & \operatorname{Tr}_{3}^{1, M} & \operatorname{Tr}_{3}^{1, W} \\
\frac{2473}{6400} & -\frac{363}{1600} & -\frac{757}{1600} & \frac{2007}{6400} \\
-\frac{363}{1600} & \frac{1407}{6400} & \frac{1441}{6400} & -\frac{349}{1600} \\
-\frac{757}{1600} & \frac{1441}{6400} & \frac{4659}{6400} & -\frac{12}{25} \\
\frac{2007}{6400} & -\frac{349}{1600} & -\frac{12}{25} & \frac{2461}{6400}
\end{array}\right)
\end{aligned}
$$




$$
\begin{aligned}
& Q\left(T_{5}^{8}, A\right)=\frac{99}{3200} \cdot\left(\begin{array}{cccc}
I^{A} & \vec{C}_{3}^{A} & \operatorname{Tr}_{3}^{A} & O^{A} \\
-1 & -1 & -1 & 1 \\
-1 & 1 & 1 & -1 \\
1 & -1 & -1 & 1
\end{array}\right)=\frac{99}{3200} \cdot\left(\begin{array}{c}
1 \\
-1 \\
-1 \\
1
\end{array}\right) \cdot\left(\begin{array}{c}
1 \\
-1 \\
-1 \\
1
\end{array}\right)^{\top}
\end{aligned}
$$

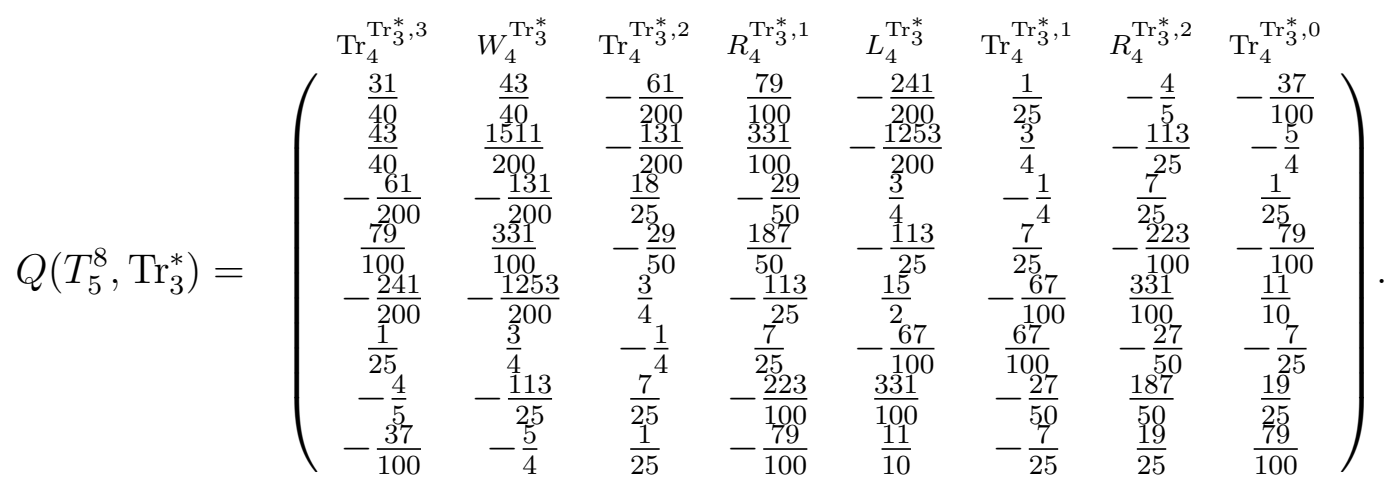

$$
\begin{aligned}
& Q\left(T_{5}^{8}, \vec{C}_{3}^{*}\right)=\left(\begin{array}{cccccccc}
R_{4}^{\vec{C}_{3}^{*}, 3} & L_{4}^{\vec{C}_{3}^{*}} & R_{4}^{\vec{C}_{3}^{*}, 2} & R_{4}^{\vec{C}_{3}^{*}, 1} & R_{4}^{\vec{C}_{3}^{*}, 23} & W_{4}^{\vec{C}_{3}^{*}} & R_{4}^{\vec{C}_{3}^{*}, 12} & R_{4}^{\vec{C}_{3}^{*}, 13} \\
\frac{391}{100} & -\frac{319}{100} & \frac{13}{20} & \frac{13}{20} & -\frac{123}{50} & \frac{87}{50} & \frac{9}{50} & -\frac{37}{25} \\
-\frac{319}{100} & \frac{367}{50} & -\frac{159}{50} & -\frac{159}{50} & \frac{7}{4} & -\frac{76}{25} & \frac{7}{4} & \frac{7}{4} \\
\frac{13}{20} & -\frac{159}{50} & \frac{389}{100} & \frac{13}{20} & -\frac{37}{25} & \frac{7}{4} & -\frac{123}{50} & \frac{9}{50} \\
\frac{13}{20} & -\frac{159}{50} & \frac{13}{20} & \frac{389}{100} & \frac{9}{50} & \frac{7}{4} & -\frac{37}{25} & -\frac{123}{50} \\
-\frac{123}{50} & \frac{7}{4} & -\frac{37}{25} & \frac{9}{50} & \frac{389}{100} & -\frac{159}{50} & \frac{13}{20} & \frac{13}{20} \\
\frac{87}{50} & -\frac{76}{25} & \frac{7}{4} & \frac{7}{4} & -\frac{159}{50} & \frac{367}{50} & -\frac{159}{50} & -\frac{159}{50} \\
\frac{9}{50} & \frac{7}{4} & -\frac{123}{50} & -\frac{37}{25} & \frac{13}{20} & -\frac{159}{50} & \frac{389}{100} & \frac{13}{20} \\
-\frac{37}{25} & \frac{7}{4} & \frac{9}{50} & -\frac{123}{50} & \frac{13}{20} & -\frac{159}{50} & \frac{13}{20} & \frac{389}{100}
\end{array}\right) . \\
& Q\left(T_{5}^{9}, 1\right)=\frac{1}{40} \cdot\left(\begin{array}{cccc}
\operatorname{Tr}_{3}^{1, L} & \vec{C}_{3}^{1} & \operatorname{Tr}_{3}^{1, M} & \operatorname{Tr}_{3}^{1, W} \\
-35 & -33 & -117 & 75 \\
-117 & 33 & 33 & -33 \\
75 & -33 & -117 & 75
\end{array}\right) \\
& Q\left(T_{5}^{9}, \vec{C}_{3}^{*}\right)=\frac{1}{5} \cdot\left(\begin{array}{cccccccc}
R_{4}^{\vec{C}_{3}^{*}, 3} & L_{4}^{\vec{C}_{3}^{*}} & R_{4}^{\vec{C}_{3}^{*}, 2} & R_{4}^{\vec{C}_{3}^{*}, 1} & R_{4}^{\vec{C}_{3}^{*}, 23} & W_{4}^{\vec{C}_{3}^{*}} & R_{4}^{\vec{C}_{3}^{*}, 12} & R_{4}^{\vec{C}_{3}^{*}, 13} \\
0 & 0 & -18 & -18 & -18 & 0 & -18 & 36 \\
-18 & 192 & 0 & 0 & 0 & -192 & 0 & 0 \\
-18 & 0 & -18 & -18 & 36 & 0 & -18 & -18 \\
-18 & 0 & 36 & -18 & 36 & 0 & -18 & -18 \\
0 & -192 & 0 & 0 & 0 & 192 & 0 & 0 \\
-18 & 0 & -18 & 36 & -18 & 0 & 36 & -18 \\
36 & 0 & -18 & -18 & -18 & 0 & -18 & 36
\end{array}\right) .
\end{aligned}
$$




$$
\begin{aligned}
& Q\left(T_{5}^{11}, 1\right)=\frac{5}{16} \cdot\left(\begin{array}{cccc}
\operatorname{Tr}_{3}^{1, L} & \vec{C}_{3}^{1} & \operatorname{Tr}_{3}^{1, M} & \operatorname{Tr}_{3}^{1, W} \\
-1 & -1 & -1 & 1 \\
-1 & 1 & 1 & -1 \\
1 & -1 & -1 & 1
\end{array}\right)=\frac{5}{16} \cdot\left(\begin{array}{c}
1 \\
-1 \\
-1 \\
1
\end{array}\right) \cdot\left(\begin{array}{c}
1 \\
-1 \\
-1 \\
1
\end{array}\right)^{\top} \\
& Q\left(T_{5}^{11}, \vec{C}_{3}^{*}\right)=\frac{1}{5} \cdot\left(\begin{array}{cccccccc}
R_{4}^{\vec{C}_{3}^{*}, 3} & L_{4}^{\vec{C}_{3}^{*}} & R_{4}^{\vec{C}_{3}^{*}, 2} & R_{4}^{\vec{C}_{3}^{*}, 1} & R_{4}^{\vec{C}_{3}^{*}, 23} & W_{4}^{\vec{C}_{3}^{*}} & R_{4}^{\vec{C}_{3}^{*}, 12} & R_{4}^{\vec{C}_{3}^{*}, 13} \\
12 & 12 & 6 & 6 & -6 & -12 & -6 & -24 \\
6 & 25 & 12 & 12 & -12 & -25 & -12 & -12 \\
6 & 12 & 27 & 6 & -27 & -12 & -6 & -6 \\
-6 & -12 & -27 & -6 & 27 & 12 & 6 & 6 \\
-12 & -25 & -12 & -12 & 12 & 25 & 12 & 12 \\
-6 & -12 & -6 & -24 & 6 & 12 & 24 & 6 \\
-24 & -12 & -6 & -6 & 6 & 12 & 6 & 24
\end{array}\right) \\
& Q\left(T_{5}^{12}, 1\right)=\frac{1}{16} \cdot\left(\begin{array}{cccc}
\operatorname{Tr}_{3}^{1, L} & \vec{C}_{3}^{1} & \operatorname{Tr}_{3}^{1, M} & \operatorname{Tr}_{3}^{1, W} \\
-1 & -1 & -1 & 1 \\
-1 & 1 & 1 & -1 \\
1 & -1 & -1 & 1
\end{array}\right)=\frac{1}{16} \cdot\left(\begin{array}{c}
1 \\
-1 \\
-1 \\
1
\end{array}\right) \cdot\left(\begin{array}{c}
1 \\
-1 \\
-1 \\
1
\end{array}\right)^{\top}
\end{aligned}
$$

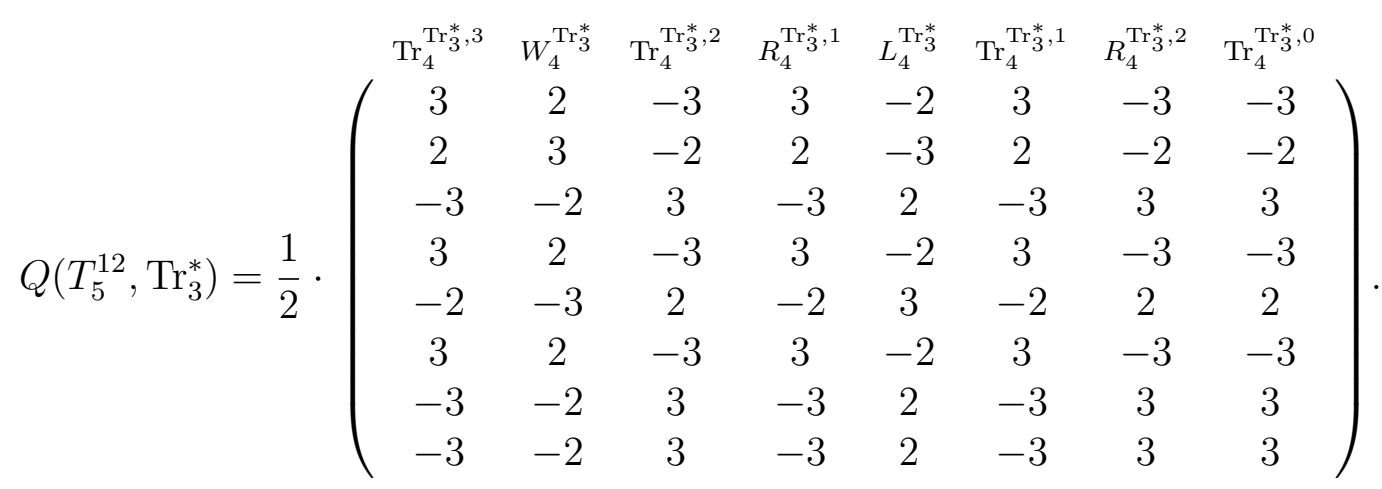

$$
\begin{aligned}
& Q\left(T_{5}^{12}, \vec{C}_{3}^{*}\right)=\frac{1}{2} \cdot\left(\begin{array}{cccccccc}
R_{4}^{\vec{C}_{3}^{*}, 3} & L_{4}^{\vec{C}_{3}^{*}} & R_{4}^{\vec{C}_{3}^{*}, 2} & R_{4}^{\vec{C}_{3}^{*}, 1} & R_{4}^{\vec{C}_{3}^{*}, 23} & W_{4}^{\vec{C}_{3}^{*}} & R_{4}^{\vec{C}_{3}^{*}, 12} & R_{4}^{\vec{C}_{3}^{*}, 13} \\
5 & 5 & 7 & 7 & -7 & -5 & -7 & -7 \\
7 & 5 & 5 & 5 & -5 & -3 & -5 & -5 \\
7 & 5 & 7 & 7 & -7 & -5 & -7 & -7 \\
-7 & -5 & -7 & -7 & 7 & 5 & 7 & 7 \\
-5 & -3 & -5 & -5 & 5 & 5 & 5 & 5 \\
-7 & -5 & -7 & -7 & 7 & 5 & 7 & 7 \\
-7 & -5 & -7 & -7 & 7 & 5 & 7 & 7
\end{array}\right)
\end{aligned}
$$




\section{A.2 Characteristic polynomials of matrices used}

$$
\begin{aligned}
& P_{Q\left(T_{5}^{7}, 1\right)}(x)=x^{4}-\frac{35}{12} x^{3} . \\
& P_{Q\left(T_{5}^{7}, \operatorname{Tr}_{3}^{*}\right)}(x)=x^{8}-30 x^{7} \text {. } \\
& P_{Q\left(T_{5}^{7}, \vec{C}_{3}^{*}\right)}(x)=x^{8}-24 x^{7} \text {. } \\
& P_{Q\left(T_{5}^{8}, 1\right)}(x)=x^{4}-\frac{55}{32} x^{3}+\frac{3450823}{10240000} x^{2}-\frac{255999851}{16384000000} x . \\
& P_{Q\left(T_{5}^{8}, A\right)}(x)=x^{4}-\frac{99}{800} x^{3} . \\
& P_{Q\left(T_{5}^{8}, \operatorname{Tr}_{3}^{*}\right)}(x)=x^{8}-\frac{2549}{100} x^{7}+\frac{675593}{5000} x^{6}-\frac{149230249}{500000} x^{5}+\frac{133434036319}{400000000} x^{4} \\
& -\frac{1980952353887}{10000000000} x^{3}+\frac{11839377144943}{200000000000} x^{2}-\frac{346051162035699}{50000000000000} x . \\
& P_{Q\left(T_{5}^{8}, \vec{C}_{3}^{*}\right)}(x)=x^{8}-\frac{951}{25} x^{7}+\frac{5084929}{10000} x^{6}-\frac{159696453}{50000} x^{5}+\frac{125755799203}{12500000} x^{4} \\
& -\frac{1934738582639}{125000000} x^{3}+\frac{700918768199117}{62500000000} x^{2}-\frac{300346502258201}{97656250000} x . \\
& P_{Q\left(T_{5}^{9}, 1\right)}(x)=x^{4}-\frac{48}{5} x^{3}+\frac{693}{100} x^{2} . \\
& P_{Q\left(T_{5}^{9}, \vec{C}_{3}^{*}\right)}(x)=x^{8}-120 x^{7}+\frac{94608}{25} x^{6}-\frac{4478976}{125} x^{5} \text {. } \\
& P_{Q\left(T_{5}^{11}, 1\right)}(x)=x^{4}-\frac{5}{4} x^{3} . \\
& P_{Q\left(T_{5}^{11}, \vec{C}_{3}^{*}\right)}(x)=x^{8}-40 x^{7}+\frac{12828}{25} x^{6}-\frac{327744}{125} x^{5}+\frac{565056}{125} x^{4} . \\
& P_{Q\left(T_{5}^{12}, 1\right)}(x)=x^{4}-\frac{1}{4} x^{3} . \\
& P_{Q\left(T_{5}^{12}, \operatorname{Tr}_{3}^{*}\right)}(x)=x^{8}-12 x^{7}+15 x^{6} \text {. } \\
& P_{Q\left(T_{5}^{12}, \vec{C}_{3}^{*}\right)}(x)=x^{8}-26 x^{7}+34 x^{6}-9 x^{5} \text {. }
\end{aligned}
$$

